\title{
Angle Sensitive Imaging: A New Paradigm for Light Field Imaging
}

\section{VIGIL VARGHESE}

School of Electrical and Electronic Engineering

A thesis submitted to the Nanyang Technological University

in partial fulfillment of the requirement for the degree of

Doctor of Philosophy

2016 



\section{Acknowledgments}

"The greater danger for most of us lies not in setting our aim too high and falling short; but in setting our aim too low, and achieving our mark."

- Michelangelo

This work would not have been possible without the vision and guidance of my thesis advisor, Dr. Shoushun Chen. His innate talent to trust his students with tasks beyond their current capabilities thereby instilling in them a confidence to aim for greater heights is one of his greatest strengths. I cannot thank him enough for his trust in me and my abilities which helped me become the person I am today. My heartfelt thanks also to Dr. Shen Zexiang, my co-supervisor for his invaluable suggestions and recommendations.

I could not have pulled off an interdisciplinary work of this nature without the help and support of my team mates and colleagues. I would like to especially thank Xinyuan Qian, Zhao Bo, Yu Hang, Tao Jin and Liang Gaozhen for their help and support. The work in NTU was made enjoyable by the numerous friends I made over the years. Special thanks to all of them - they know who they are!

I would also like to specially thank School of Electrical and Electronics Engineering of NTU for awarding me the postgraduate scholarship and also to VIRTUS IC Design Centre of Excellence.

Special thanks and gratitude to my parents for always being there as a constant source of motivation and support. This thesis is dedicated to them for teaching me to dream and making me believe that dreams do come true through hard work. 



\section{Contents}

Acknowledgments

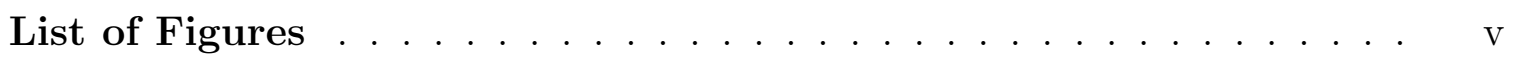

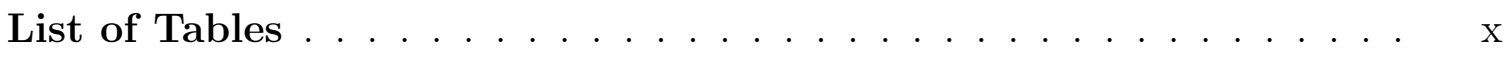

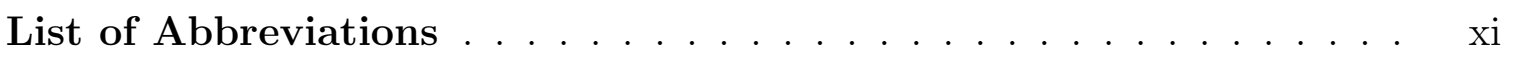

Abstract ................................

1 Introduction 1

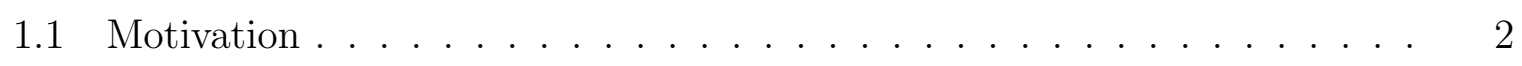

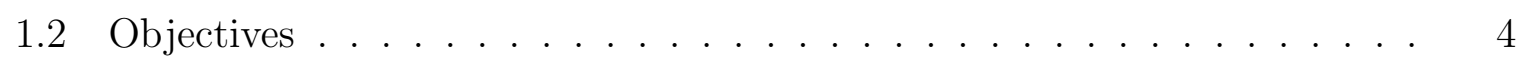

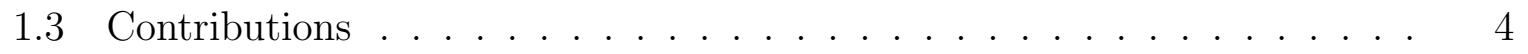

1.4 Thesis Organization . . . . . . . . . . . . . . . . . 5

2 From Light Field Imaging to Angle Sensitive Imaging $\quad 7$

2.1 Light Fields . . . . . . . . . . . . . . . . . . . . 7

2.2 Parameterization of Light Fields . . . . . . . . . . . . . . . . . . 9

2.3 Angle Sensitive Imaging _ . . . . . . . . . . . . . . . . . . . . . . . . 10

2.4 Advantages of Angle Sensitive Imaging . . . . . . . . . . . . . . . . 11

3 Review of the State-of-the-Art 14

$3.1 \quad$ Plenoptic Imaging . . . . . . . . . . . . . . . . . . . . . . . . . . . . . . 17

3.2 Focused Plenoptic Imaging . . . . . . . . . . . . . . . . . . . . . 19 
3.3 Talbot Imaging . . . . . . . . . . . . . . . . . . . . . . . . . 21

3.4 Enhanced Talbot Imaging _ . . . . . . . . . . . . . . . . . . 25

4 Angle Sensitive Imaging using Metal Masks $\quad 27$

4.1 Quadrature Pixel Cluster . . . . . . . . . . . . . . . . 27

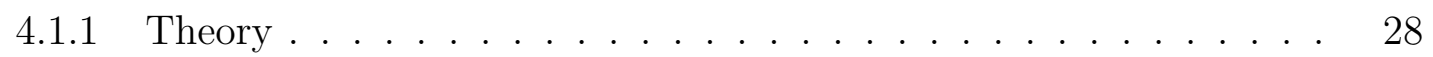

4.1 .2 Simulations . . . . . . . . . . . . . . . . . 32

4.2 Track-and-Tune Angle Detection Technique . . . . . . . . . . . . . 36

4.2 .1 Sensor Architecture . . . . . . . . . . . . . . . . 38

4.2 .2 Results and Discussion . . . . . . . . . . . . . . . . . . 42

4.3 Enhanced Quadrature Pixel Cluster . . . . . . . . . . . . . . . . . 47

4.4 Multi-Finger Pixel Design _. . . . . . . . . . . . . . . . . . 50

4.5 Orthogonal Multi-Fingered Pixels . . . . . . . . . . . . . . . . . 53

4.6 Antisymmetric Multi-Fingered Pixels . . . . . . . . . . . . . . 56

5 Polarization-Incident Angle Sensitive Imaging $\quad 57$

5.1 Combining Polarization Pixels with Quadrature Pixel Cluster . . . . . . 64

5.2 Prototype Angle Sensitive Polarization Sensor . . . . . . . . . . . . . 65

5.3 Test Setup and Testing Methodology . . . . . . . . . . . . . . . 67

5.4 Experimental Results . . . . . . . . . . . . . . . . . . 68

5.4.1 Characterization of Polarization Pixels . . . . . . . . . . 68

5.4.2 Comparing Polarization, Talbot and QPC Pixel responses . . . . 70

5.4.3 Angle Detection using Polarization and QPC Pixels . . . . . . . . 71

5.5 Discussion . . . . . . . . . . . . . . . . . . 73

5.5.1 Design Limitations . . . . . . . . . . . . . . . . . . . 73

5.5 .2 Experimental Limitations . . . . . . . . . . . . 74 
6 Angle Sensitive Imaging: Evaluation and Applications $\quad 77$

6.1 Framework for Evaluating Angle Sensitive Imaging . . . . . . . . . . . . 77

6.1 .1 Spatial Resolution . . . . . . . . . . . . . . . 78

6.1.2 Angular Resolution . . . . . . . . . . . . . . . . . 81

6.1 .3 Light Transmittance . . . . . . . . . . . . . . . . . . . . 82

6.1 .4 Angle Sensitivity . . . . . . . . . . . . . . . . . . 83

6.1.5 Wavelength Sensitivity . . . . . . . . . . . . . . . . . 84

6.1.6 Polarization Sensitivity . . . . . . . . . . . . . . . 86

6.1 .7 Putting it all Together . . . . . . . . . . . . 87

6.2 Applications of Angle Sensitive Imaging . . . . . . . . . . . . . . . . . 88

6.2.1 Fast Response Auto-Focus Systems . . . . . . . . . . . . . . . . . 88

6.2 .2 Depth Estimation . . . . . . . . . . . . . . . . 96

6.2 .3 Post Capture Image Refocus . . . . . . . . . . . . . . . . . . . . . 98

7 Conclusion and Future Research 103

7.1 Conclusion . . . . . . . . . . . . . . . . . . 103

7.2 Summary of Contributions . . . . . . . . . . . . . . . . . 105

7.3 Future Research and Potential Applications . . . . . . . . . . . . . . . 105

$\begin{array}{ll}\text { Author's Publications } & 108\end{array}$

$\begin{array}{ll}\text { Bibliography } & 110\end{array}$ 


\section{List of Figures}

2.1 Representation of a ray in $4 \mathrm{D}$ light field space [46]. . . . . . . . . . . . 9

2.2 From light filed imaging to angle sensitive imaging. . . . . . . . . . . . . 10

3.1 Microlens array in a multi-aperture image sensor [17] . . . . . . . . . . 18

3.2 Principle of plenoptic imaging $[60] \ldots \ldots \ldots$. . . . . . . . . . . 19

3.3 Sub-aperture image in plenoptic imaging [60]. . . . . . . . . . . . . . . . 19

3.4 Two sub-aperture images formed by collecting highlighted pixels from the same position underneath each microlens [60]. . . . . . . . . . . 20

3.5 Image formation process in a focused plenoptic camera (figure from [25]). 20

3.6 Figure showing the physical structure of Talbot pixels and self-image formation

3.7 Sample response produced by Talbot pixels of figure 3.6 as a function of incidence angle variation [88]. . . . . . . . . . . . . . . . . . . . . . 24

3.8 Figure showing three of the enhaced Tablot pixels proposed in [72] (figures from $[72]) \ldots \ldots \ldots \ldots \ldots \ldots \ldots \ldots \ldots \ldots \ldots \ldots \ldots \ldots$

4.1 Physical structure of angle sensitive quadrature pixel cluster. . . . . . . . 28

4.2 2D view of quadrature pixel cluster along the X direction. . . . . . . . 29

4.3 Difference in unshaded area as a function of incident angle for two adjacent photodiodes in a quadrature pixel cluster. . . . . . . . . . . . . 
4.4 FDTD simulations showing electric field profiles for a pair of pixels in a quadrature pixel cluster. . . . . . . . . . . . . . . . . 33

4.5 Variation in intensity as a function of incident angle for two adjacent photodiodes in a quadrature pixel cluster. . . . . . . . . . . . . . . 34

4.6 Quadrature pixel response as a function of incident angle for varying metal thickness. . . . . . . . . . . . . . . . . .

4.7 Quadrature pixel response as a function of incident angle for varying metal widths. . . . . . . . . . . . . . . . . . 36

4.8 FDTD simulation of difference response produced by Talbot pixels $(\lambda=$

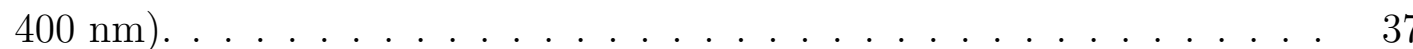

4.9 Technique illustrating angle detection using Talbot and QPC pixel responses. 38

4.10 Sensor architecture along with chip microphotograph. . . . . . . . . . . 39

4.11 Sensor schematic showing the various structural components of the fabricated sensor. The various pixel types and their ideal responses are also shown (refer Table 4.1 for different pixel types). . . . . . . . . . . . . . . 40

4.12 Conceptual diagram of the test setup. . . . . . . . . . . . . . . . 43

4.13 Plot of measured pixel responses as the incidence angle is varied from $-45^{\circ}$ to $+45^{\circ}$. Different pixel types and ideal responses are shown in Fig. 4.11 and Table $4.1 \ldots \ldots \ldots \ldots$. . . . . . . . . . . . . . . . . . . 44

4.14 Figure showing measurement of angle from experimental data. . . . . . . 45

4.15 Physical structure of enhanced angle sensitive quadrature pixel cluster. . $\quad 47$

4.16 Response of enhanced QPC pixels and their difference. . . . . . . . . . . 48

4.17 Comparing angle sensitivity of QPC and enhanced QPC pixels. . . . . . 49

4.18 Enhanced QPC pixel with microlens [39] . . . . . . . . . . . . . . . 49

4.19 Single multi-finger pixel. . . . . . . . . . . . . . . . . . . . 50 
4.20 Response produced by a horizontal multi-finger pixel for vertical light angle variation. . . . . . . . . . . . . . . . . . 51

4.21 Physical structure of orthogonal multi-finger pixels. . . . . . . . . . 53

4.22 Multi-finger angle sensitive image sensor. . . . . . . . . . . . . . . . . 54

4.23 Response of orthogonal multi-finger pixels. . . . . . . . . . . . . 55

4.24 Antisymmetric MF pixel structure and response. . . . . . . . . . . . . 56

5.1 Figure showing electric-field vector orientation of randomly polarized light (a) and partially polarized light (b) with its major component oriented along the $90^{\circ}$ axis. . . . . . . . . . . . . . . . . 58

5.2 Figure showing unpolarized light being horizontally polarized by a vertical polarization grating. . . . . . . . . . . . . . . . . 61

5.3 Physical structure of single-layer polarization pixels. . . . . . . . . . . . 62

5.4 Electric field intensity versus incidence angle variation for $0^{\circ}$ (a) and $90^{\circ}$ (b) polarization pixels under unpolarized light, $90^{\circ}$ or vertically polarized light and $0^{\circ}$ or horizontally polarized light. . . . . . . . . . . . .

5.5 Electric field intensity versus incidence angle variation of unpolarized light on $0^{\circ}$ polarization pixel as comapred with $60^{\circ}$ polarized light on $0^{\circ}$ and $90^{\circ}$ polarization pixels along with the summed response of $0^{\circ}$ and $90^{\circ}$ polarization pixels

5.6 Electric field intensity versus incidence angle variation of unpolarized light on $0^{\circ}$ polarization pixel as comapred to the $\mathrm{QPC}$ pixel response. . . . . .

5.7 Microphotograph showing sensor architecture along with the prominent pixel types in the sensor. . . . . . . . . . . . . . . . .

5.8 Pixel voltage versus incidence angle variation for $0^{\circ}$ (a) and $90^{\circ}$ (b) polarization pixels under unpolarized light, $90^{\circ}$ or vertically polarized light and $0^{\circ}$ or horizontally polarized light. 
5.9 Pixel voltage versus incidence angle variation for $0^{\circ}$ and $90^{\circ}$ polarization pixels under $90^{\circ}$ or vertically polarized light (a) and $0^{\circ}$ or horizontally

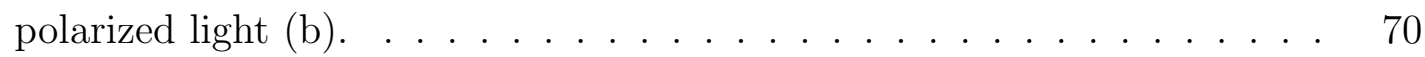

5.10 Pixel voltage versus incidence angle variation for $0^{\circ}$ and $90^{\circ}$ polarization pixels under unpolarized light. . . . . . . . . . . . . . . . . . . . 71

5.11 Pixel voltage versus incidence angle variation of differential quadrature pixel cluster (QPC), differential Talbot effect based angle sensitve pixel (ASP) and $90^{\circ}$ polarization pixel.

5.12 Pixel voltage versus incidence angle variation of differential quadrature pixel cluster $(\mathrm{QPC})$ and $90^{\circ}$ polarization pixel illustrating the angle detection technique. . . . . . . . . . . . . . . 73

6.1 Spatial resolution of various light field imaging techniques. . . . . . . . . 80

6.2 Angular resolution of various angle sensitive pixels. . . . . . . . . . . . . 81

6.3 Light transmittance for various angle sensitive imaging pixels. 'Enhanced Talbot 1' uses amplitude grating with interleaved N+/P-sub diode, 'Enhanced Talbot 2' uses phase grating with N-well/P-sub diode, 'Enhanced Talbot 3' uses phase grating with interleaved N+/P-sub diode, 'Enhanced Talbot 4' uses amplitude grating with interleaved $\mathrm{P}+/ \mathrm{N}$-well diode. . . . 83

6.4 Angle sensitivity of various angle sensitive imaging pixels. . . . . . . . . . 84

6.5 Wavelength response of various angle sensitive pixel types. . . . . . . . . 85

6.6 Polarization response of various angle sensitive pixel types. . . . . . . . 87

6.7 Principle of auto focus systems $([14]) \ldots \ldots \ldots \ldots$

6.8 Principle of auto focusing (from [14]) $\ldots \ldots \ldots \ldots$

6.9 PDAF system $($ from $[39]) \ldots \ldots \ldots \ldots$. . . . . . . . . . . . . . 90

6.10 In-Focus image captured using MF sensor and its 1D profile. . . . . . . . 91

6.11 Gradual change in image focus (pixels encounter converging angles). . . . 91 
6.12 1D profile of figures $6.11(\mathrm{a})-6.11(\mathrm{f}) \ldots \ldots . \ldots 9 . \ldots . \ldots 92$

6.13 Plot highlighting the difference between horizontal and vertical MF pixel

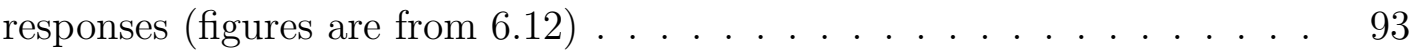

6.14 Gradual change in image focus (pixels encounter diverging angles). . . . . 93

6.15 1D profile of figures $6.14(\mathrm{a})-6.14(\mathrm{f}) \ldots \ldots . \ldots . \ldots 94$

6.16 Plot highlighting the difference between horizontal and vertical MF pixel responses (figures are from 6.15). . . . . . . . . . . . . . . . . 95

6.17 Figure to illustrate depth estimation process in multi-aperture image sensors $[4] . \ldots \ldots \ldots \ldots$

6.18 Directional information contained in the light rays when the object is "Near", "In-focus" and "Far" from the lens [88]. . . . . . . . . . . . . . 97

6.19 Three white bars with one of the three in focus in each image and its 1D profile along the marked horizontal line. . . . . . . . . . . . . . . . . . . 99

6.20 Image blur profiles. . . . . . . . . . . . . . . . . . . . . . . 101

6.21 Image with a small amount of defocus and its refocused image $(\sigma=9)$. . 101

6.22 Image with a large amount of defocus and its refocused image $(\sigma=13) . \quad$. 102 


\section{List of Tables}

4.1 Pixel Types in a Macro Pixel _. . . . . . . . . . . . . . . . . . . 41

4.2 Important Sensor Parameters . . . . . . . . . . . . . . . . . . . . . 42

5.1 Important Sensor Parameters of Polarization Sensor . . . . . . . . . . . . 68

6.1 Defocus and Refocus Measure . . . . . . . . . . . . . . . . . . 102 


\section{List of Abbreviations}

$\begin{array}{ll}\text { ADC } & \text { Analog-to-Digital Converter } \\ \text { APS } & \text { Active Pixel Sensor } \\ \text { CCD } & \text { Charge Coupled Device } \\ \text { CDAF } & \text { Contrast Detection Auto Focus } \\ \text { CDS } & \text { Correlated Double Sampling } \\ \text { CMOS } & \text { Complementary Metal-Oxide-Semiconductor } \\ \text { DSLR } & \text { Digital Single Lens Reflex } \\ \text { FDTD } & \text { Finite Difference Time Domain } \\ \text { FF } & \text { Fill Factor } \\ \text { FPN } & \text { Fixed Pattern Noise } \\ \text { MF } & \text { Multi-Finger } \\ \text { PD } & \text { Photodiode } \\ \text { PDAF } & \text { Phase Detection Auto Focus } \\ \text { QPC } & \text { Quadrature Pixel Cluster } \\ \text { SNR } & \text { Signal to Noise Ratio }\end{array}$




\section{Abstract}

Imaging is a process of mapping information from higher dimensions of a light field into lower dimensions. Conventional cameras do this mapping into two dimensions of the image sensor array. These sensors lose directional information contained in the light rays passing through the camera aperture as each sensor element (called a pixel) integrates all the light rays arriving at its surface. Directional information is lost and only intensity information is retained. This work is in pursuit of a method to decouple this link and enable image sensors to capture both intensity and direction without sacrificing much of the spatial resolution as the existing techniques do.

Numerous applications have been demonstrated in the past that benefit from the additional directional information with the passive depth estimation being an obvious one. Others include multi-view point rendering, extended depth of field imaging, post capture image refocus, visibility in the presence of partial occluders and 3D scene reconstruction. This work concentrates on the ubiquitous issue of capturing high resolution light fields, consciously relegating the potential applications as simple software solutions to the designed hardware (image sensor). Once the 4D information is available, suitable modifications to the data set result in its application to diverse areas.

Existing techniques that align their goals with this work have a severe shortcoming in terms of the achievable spatial resolution. The trade-off is that of the spatial versus directional resolution. One cannot be increased without affecting the other. This work attempts to find an optimum solution that maximizes spatial resolution without affecting the quality of the directional information captured thereby ensuring that sufficient directional information is available for computational post processing techniques. 
This work builds heavily on the theoretical premise laid down by the earlier work on multi-aperture imaging. Practical aspects are modeled on the diffraction based Talbot effect. The solution falls into a general category of sub-wavelength apertures and is a one-dimensional case of the same. We explore other alternative solutions such as differential quadrature pixels, polarization pixels, multi-finger pixels and combinations of these to effectively capture the angular information of light by consuming only a very small imager area. We establish the capabilities of our technique through rigorous testing of individual sensing elements and the image sensor as a whole. Our solution enables a rich set of applications among which are fast response auto-focus camera systems and single-shot passive 3D imaging. 


\section{Chapter 1}

\section{Introduction}

The word "photography" was coined in 1839 by Sir John Frederick William Herschel, which derives its root from the Greek words "photos" (meaning "light") and "graphe" (meaning "drawing"). The year 1839 is also widely regarded as the birth year of practical photography [34].

A new era of photography began in 1969 with the invention of charge coupled devices (CCD) by Willard Boyle and George E. Smith at AT\&T Bell Labs. First prototype camera with the CCD was built by Steven Sasson in 1975 at Eastman Kodak [1]. Commercially released in 1990 as Dycam Model 1 (a.k.a Logitech Fotoman), they were to become the backbone of photography industry. With the CMOS manufacturing process becoming more stable by the early 1990s, interest in CMOS image sensors were renewed. Gradually by late 1990s, CMOS image sensors began to challenge the CCD sensors, first in the low end imaging applications and then at high end digital photography. CCD sensors are still used widely in many scientific applications that require high efficiency and SNR, but CMOS sensors have become ubiquitous due to its low cost and use of standard CMOS fabrication processes.

The continuous miniaturization of micro fabrication processes and its cost reduction over the years has enabled many researchers to rethink the traditional form of image sensing. It is no longer restrictive to consider imaging to be a $2 \mathrm{D}$ conversion process. 
As we will explore later in this thesis, miniaturization has led to use of micro-lenses and gratings directly on top of image sensor pixels to alter the information they capture.

\subsection{Motivation}

We live in a three dimensional world, yet the images we capture are limited to only two dimensions. In capturing an image from a real scene onto an image sensor we sample only the two spatial dimensions of $\mathrm{x}$ and $\mathrm{y}$. The third dimension, $\mathrm{z}$, which enables the depth perception is lost. Capturing the full 3D information from the scene of interest allows for some novel reconstruction techniques that enables creation of depth maps, post capture refocus and multi-viewpoint rendering. Although numerous multi-sensor, multiexposure techniques have been successful in capturing enough information to enable 3D reconstruction, they become severely limited for dynamic scenes and scenes under low light conditions.

An ideal solution to enable full 3D information capture under all imaging conditions would be one that uses single sensor with a single exposure to capture the image. Such a solution would require auxiliary optical components in the focal plane of the image sensor to extract additional information from the imaged scene. Just as addition of color filters enable capture of wavelength information from incident light, enabling color photographs, optical components such as micro-lenses and metallic gratings/masks enable capture of angle information enabling light field photography. Capturing light angle opens up new avenues in areas such as 3D image capture, post capture image refocusing and depth map computation in addition to the whole range of image processing capabilities that 4D light field information provides.

This work builds upon the previous 100 years of efforts to capture a rich light field from the visual scene. The first practical design for creating a light field camera was laid down by the Nobel prize winning Gabriel Lippmann (although the Nobel prize was for his 
works on photographic reproduction of color). This followed decades of experimentation that were limited to laboratory settings due to the cost of the components involved and their delicate assembly.

The field went through a renaissance in late 1990's and early 2000's with a slew of techniques being proposed, vastly advancing the state of the art. A brief overview of the techniques proposed during this era can be found in chapter 3 .

Through this work we propose a slew of techniques for light angle detection that significantly enhances the state-of-the-art. A common theme that runs through this thesis is the optimization for the spatio-angular resolution problem. The plenoptic cameras (details in chapter 3) that resulted from the works of Ren $\mathrm{Ng}$, and which was later commercialized as Lytro camera was plagued by this problem. The technique inherently sacrifices spatial resolution for angular resolution. This tight coupling between the two parameters meant that we could not increase one without decreasing the other.

The focused plenoptic camera (details in chapter 3) was based on the same technique as that of the plenoptic camera but provides the flexibility to trade-off some of the angular resolution for an increased spatial resolution. As this technique is based on the plenoptic camera there still is some loose coupling between the spatial and angular resolution.

An alternative technique based on wavelength scale diffraction gratings, utilizing an effect called Talbot effect, came into the scene in mid 2009. This technique could capture local angle information present in out-of-focus images, yet, when the images are in focus, they also capture local intensity information at higher spatial resolution. The technique presented an alternative means for light field capture and broke the traditional trade-off that existed between the spatial and angular resolution. A limiting factor of the technique was the need for a large number of pixels for determining the local angle information. Although there was no spatio-angular resolution trade-off, significant spatial information was sacrificed for capturing a wide angular information. 
The techniques presented in this thesis enable fabrication of light field image sensors that are practical (maximize spatio-angular resolution), robust (no post processing after fabrication) and cost effective (use conventional CMOS fabrication processes).

\subsection{Objectives}

The broad scope of this thesis is to advance the state-of-the-art in light field image capture by proposing techniques based on angle sensitive imaging. The four main objectives are:

(i) Investigation of angle dependent behavior of pixels based on wavelength scale gratings and examining their characteristics.

(ii) Theoretical analysis and design of new class of angle sensitive imaging techniques with improved spatio-angular resolution.

(iii) Explore alternative strategies for hybrid imaging, such as hybrid polarizationincident angle sensitive imaging.

(iv) Demonstrate imaging applications using designed angle sensitive sensors that outperform conventional image sensors for certain applications.

\subsection{Contributions}

The main contributions of this thesis are:

(i) Design of wavelength scale metallic structures for incident light angle detection and evaluation of the same through FDTD (Finite Difference Time Domain) simulations.

(ii) Formalizing an explanation for the pixel level angle detection within the framework of light field representation and investigating the devices developed in this thesis through the framework. 
(iii) Design of prototype sensors with multiple pixel types that utilize wavelength scale metallic structures at their focal plane for detecting incident angle of light.

(iv) Design of a full scale image sensor to bring together the angle detecting pixels and the light field framework to demonstrate applications of $3 \mathrm{D}$ imaging and fast response auto-focus.

\subsection{Thesis Organization}

The rest of the thesis is organized as follows:

Chapter 2 introduces some background required to appreciate the materials in this thesis. It starts with a definition of the light field and then goes on to explain its parameterization and dimensionality reduction. The chapter also links the concepts of light filed imaging with angle sensitive imaging and lists some of the advantages of angle sensitive imaging.

Chapter 3 reviews the state-of-the-art work in the field of light field imaging and angle sensitive imaging. We start with a brief description of the scope of the work carried out and briefly go through 100 years of efforts put into capturing the light field information. We perform an in-depth review of the plenoptic, focused plenoptic, Talbot and enhanced Talbot imaging techniques which are predecessors to the techniques developed here.

Chapter 4 introduces four angle sensitive pixel types that use metal masks on top of the pixels to make their response sensitive to incident light angle. We formalize a mathematical description of the angle sensitive behavior of the designed pixels and illustrate the various design trade-offs. We examine the angle sensitive behavior through finite difference time domain (FDTD) simulation and verify this with the fabricated prototype sensors.

Chapter 5 introduces a hybrid polarization-incident light angle sensitive pixel that produces incident angle sensitive and polarization sensitive response. We start with 
introduction of the polarization property of light and illustrate its various applications. We then devise a scheme to determine angle sensitive response that is independent of the local polarization state of light. We verify our hypothesis through FDTD simulations and tests on a fabricated prototype sensor.

Chapter 6 binds together the concepts discussed in the thesis by providing a framework for evaluating angle sensitive imaging. It also demonstrates three applications using the designed angle sensitive sensor that touches upon areas such as camera auto-focus systems, depth map estimation and image refocusing.

Chapter 7 concludes the thesis by reiterating the contributions that arose as a result of the work carried out in this thesis. We also touch upon some topics and ideas that could be explored to further enhance this work or to develop new systems for computational imaging. 


\section{Chapter 2}

\section{From Light Field Imaging to Angle Sensitive Imaging}

This chapter provides the background information necessary to appreciate this thesis. It starts with an introduction to concepts related to light fields and facilitates a transition from light field imaging to angle sensitive imaging. It concludes by exploring the advantages angle sensitive imaging techniques offer in comparison to light field imaging.

\section{$2.1 \quad$ Light Fields}

The concept of light fields was first introduced by Faraday in [16] and was formalized by Arun Gershun through his work in [26] and later by Moon and Spencer in [51]. These concepts were incorporated into computer vision through the works of Adelson and Bergen [3] and was made feasible to use through the works of Levoy et al. [46], Gortler et al. [30] and Isaksen et al. [36].

Light field at any point in space can be described by a collection of rays from all other points in space to that particular point. A light field can be mathematically described by a seven dimensional parameterized function, know as the plenoptic function [3]. This seven dimensional function describes light field in terms of intensity variations along the $\mathrm{x}$ and $\mathrm{y}$ directions, where $(\mathrm{x}, \mathrm{y})$ are the spatial co-ordinates of an imaginary plane placed 
at a unit distance from the pupil (one can equally consider the spherical co-ordinates $\theta$ and $\phi)$, at all times $(\mathrm{t})$, for all wavelengths $(\lambda)$ and for all viewing directions $\left(\mathrm{V}_{\mathrm{x}}, \mathrm{V}_{\mathrm{y}}\right.$ and $\mathrm{V}_{\mathrm{z}}$ ). The 7D plenoptic function is given by Eq. (Eq. 2.1). This equation neglects the effects of polarization as the human visual system is incapable of detecting the same.

$$
\mathrm{P}\left(\mathrm{x}, \mathrm{y}, \lambda, \mathrm{t}, \mathrm{V}_{\mathrm{x}}, \mathrm{V}_{\mathrm{y}}, \mathrm{V}_{\mathrm{z}}\right)
$$

It is impossible to measure the complete $7 \mathrm{D}$ plenoptic function for any system. The function however serves as a means to examine the potential information available to an observer. The plenoptic function can be simplified for easier measurements without much loss of generality [46] [30]. For a static scene (no variations with time, "t") with monochromatic illumination (constant wavelength, " $\lambda$ "), the 7D function can be reduced to a $5 \mathrm{D}$ one, given below:

$$
\mathrm{P}\left(\mathrm{x}, \mathrm{y}, \mathrm{V}_{\mathrm{x}}, \mathrm{V}_{\mathrm{y}}, \mathrm{V}_{\mathrm{z}}\right)
$$

This $5 \mathrm{D}$ function can be further reduced to a 4D one by considering the fact that the radiance along a ray does not change unless blocked [46] [30]. This $4 \mathrm{D}$ representation can be used to completely describe any visual scene around us. A ray in free space is characterized by position, direction and radiance. Position represents the location of the object from where the ray emerged. Direction represents the angle at which the ray strikes the imaging plane (sensor) and radiance represents the intensity of the ray. We do not loose any generality in reducing the light field to 4D as we will only be considering the field inside the camera - from aperture to the sensor. The rich information contained within these confines have been proven to be very useful for light field applications [59].

Conventional image sensors capture only the average intensity of the rays that terminate at a particular sensing element (pixel). The directional information is lost. Capturing the directional information offers flexibility in creating synthetic photographs by using ray tracing or image rendering techniques. This flexibility comes from the fact that 
the devised algorithms could be formulated independent of scene geometry or illumination that restrict the current techniques. The image formation process can be considered as extracting a $2 \mathrm{D}$ slice from a 4D light field.

\subsection{Parameterization of Light Fields}

A 3D object could be considered bound within a hypothetical cube for ease of representation. Each face of the cube represents a different plane. We can look at the object from any one of the cube faces - front, back, up, down or either of the two sides. Consider an object placed in such a hypothetical cube. This cube represents the convex hull of the object. The object can be visually represented by considering the light rays that leave the object and intersect the cube. We can represent any object using a light field. A light field accounts for all the rays from the object that intersect the hypothetical cube and the radiance along each ray.

Fig. 2.1 shows the representation of a ray in $4 \mathrm{D}$ light field space. The first plane $(\mathrm{u}$, v) indicates the position of the light ray emerging from an object, while the second plane $(\mathrm{s}, \mathrm{t})$ shows the direction of the light ray. Within a camera body one can consider the $(\mathrm{u}, \mathrm{v})$ plane to be the lens aperture and the $(\mathrm{s}, \mathrm{t})$ plane to be the image sensor plane. The planes are normal to the z-axis.

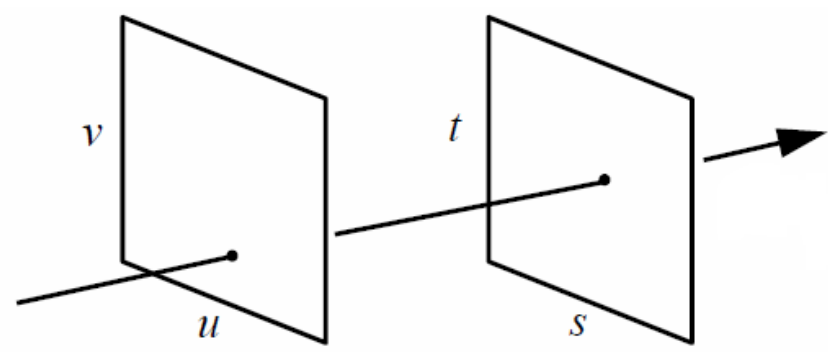

Figure 2.1: Representation of a ray in 4D light field space [46].

Although the light-slab representation is simple to use, it is limited by uneven sam- 
pling density and uncertainty in reliably extrapolating the viewing position in the presence of occluders.

\subsection{Angle Sensitive Imaging}

Extending the concept of light-slab further, now imagine a sensor ( 2 dimensional imaging plane) placed behind the $(\mathrm{s}, \mathrm{t})$ plane as shown in Fig. 2.2(a). From the perspective of a pixel in the sensor, all that matters is the angle each ray makes with it (Fig. 2.2(b)). Hence, our aim in this work is to determine the angle of incident light ray at the pixel level instead of its direction.

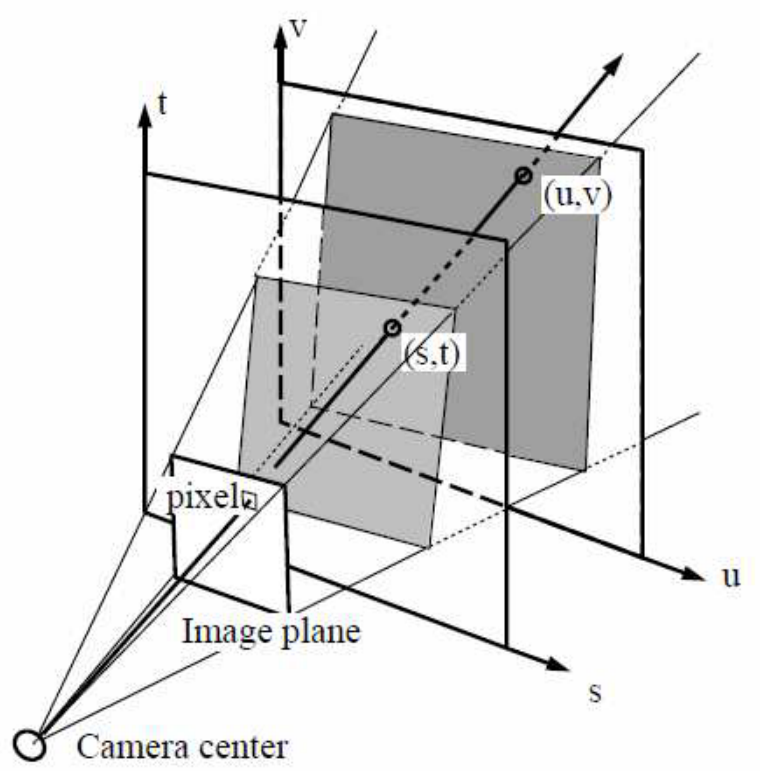

2.2.a: $4 \mathrm{D}$ light field from the perspective of an image sensor $[30]$.

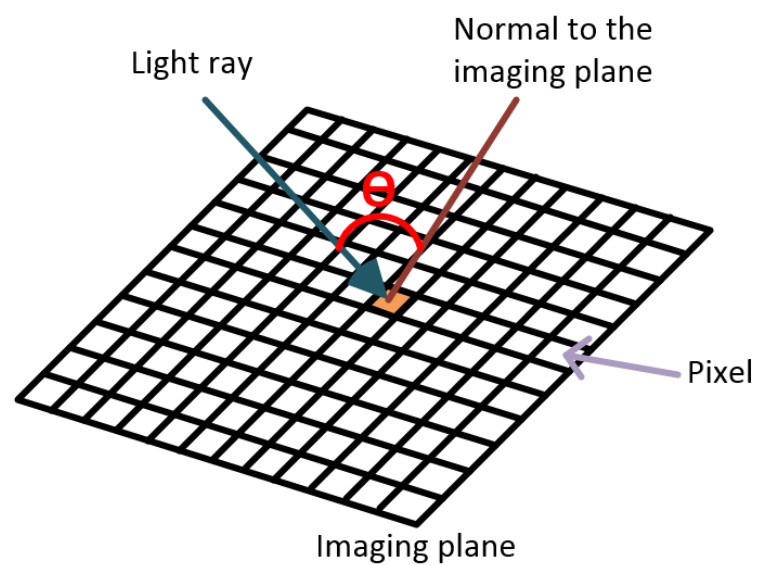

2.2.b: Angle made by the light ray with an imaging element (pixel).

Figure 2.2: From light filed imaging to angle sensitive imaging.

This representation marks a transition from the traditional ray based light field capturing techniques to the angle based light field capturing techniques, which we term angle sensitive imaging. 


\subsection{Advantages of Angle Sensitive Imaging}

Much of the past literature is scattered with techniques for light field capture that were bulky, expensive and complicated. Miniaturization of IC fabrication processes have brought about a positive change in the field of light field imaging starting with the initial approaches of Ren Ng [59] and Keith Fife [18] and advanced by Albert Wang et al. [88] through his work on Talbot imaging. By making microlenses redundant, the technique of Talbot imaging truly champions the miniaturization mindset. Some of the advantages that angle sensitive imaging techniques bring to the table are listed below:

(i) Portability: Techniques such as camera arrays, camera motion, camera gantry, multi-aperture imaging using relay lenses, were extremely bulky and remained limited to laboratory settings. Although these initial systems did a good job of promoting the usefulness of light field imaging techniques, they were limited by the technologies of their time and never reached the common man. With the advancement of technology, the multi-aperture system, which is essentially the miniaturized version of the earlier multi-camera setup became portable enough to be developed in large scale and created an industry of its own. The pioneers of these systems Ren Ng who developed the miniaturized plenoptic camera and Christian Perwass who developed the focused plenoptic camera went onto found Lytro and Raytrix respectively. Angle sensitive imaging will further this trend by providing portable solutions that can be incorporated into the high end DSLR systems or the low cost mobile camera systems. There is still some ground work to be done before angle sensitive imaging becomes ubiquitous, notably in developing robust angle sensitive pixel structures (which we address in this thesis) and novel computational techniques (we only dabble a lit bit here) that use the information provided by these sensors for practical applications. 
(ii) Manufacturability: The multi-aperture systems use microlenses at the focal plane of the camera and need specialized fabrication processes for manufacturing these, inadvertently increasing the system cost. Multiple pixels behind each microlens necessitates high precision alignment with the image sensor array. Angle sensitive imaging overcomes these issues as they can be fabricated using standard CMOS fabrication processes without any additional post processing.

(iii) Scalability: The multi-aperture technique trades spatial resolution for angle information. For a fixed resolution image sensor, spatial resolution could be increased by increasing the number of microlenses while subsequently reducing the angular resolution. On the other hand, if one desires to increase the angular resolution, each microlens has to employ a substantial number of pixels thereby reducing the spatial resolution. For this technique to be truly useful we will require a large imager array which will introduce problems of its own - large readout time (increases dark current), higher power consumption (due to the bigger source followers needed to drive the long column bus) and a host of other issues. When a particular object plane is in complete focus, there is still a reduction in the spatial information without any useful angular information. Angle sensitive techniques do not have this trade-off and can produce images with very high spatial resolution for in-focus scenes. The amount of angular information captured is directly proportional to the amount of defocus in a scene and imposes a limit only on the recoverable spatial information (aliasing prevents recoverable information for scenes with large defocus).

(iv) Programmability: Since angle sensitive image sensors are a planar array of pixels without microlenses, they can be combined with other techniques developed for conventional imagers. These techniques include high dynamic range imaging (double exposure, pixels having assorted exposure times for encoding motion and focus) and low light imaging (log pixels) among others. 
(v) Simplicity: Light field techniques using large camera arrays or camera motion extract light field information from data contained in a number of images. Needless to say, considerable processing time and effort has to be expended to gather the light field data. In multi-aperture techniques the processing is somewhat simple, in that, they resort to ray tracing (i.e. shift-and-add) to extract the light field data. The angle sensitive imaging technique makes data extraction extremely simple for simple applications since local pixels (pixels in a neighborhood) contain local light field (angle information) data. This leads to extremely simple methods for quick depth information or defocus information that other techniques find hard to process. This helps in real time applications where low level fast processing is extremely important. High level light field data can then be extracted using other computational techniques such as Gabor filtering or Deconvolution. This is akin to the low-level, high-level vision processing employed by the human brain. 


\section{Chapter 3}

\section{Review of the State-of-the-Art}

Before delving into the large body of work related to computational image capture, particularly light field capture, it is important to distinguish between the different subfields [100] of computational imaging.

Computational cameras relate to all the techniques that modify the camera structure (optics, illumination, pixels, etc.,) in order to capture a rich representation of the world view.

Computational photography (also referred as epsilon photography [63]) relates to capturing multiple images, using either single or multiple cameras and employing computational algorithms to extract useful information from them. In this case the individual images could be used as such or an augmented image could be generated from multiple images.

Computational imaging relates to techniques that focus on the process of image capture and are not usually limited to the restricted camera body (camera setup, external illumination, etc.,).

The differences between the three are subtle and usually overlapping, and there is no consensus in literature about what classification to use. It is foolhardy to strictly adhere to this classification, but at least for the purposes of this thesis establishing these bounds 
will help to constrain our thinking in terms of the best solution for a particular problem within a particular realm.

For the work in this thesis we focus our attention to techniques that modify the image sensor pixels in order to capture the light field and appropriately these classes of techniques fall into the domain of computational cameras. We passingly mention techniques from computational photography and computational imaging that aid in light field capture without explicitly distinguishing between them. We hope to convince the reader about our choice of selection and convey the advantages that computational cameras offer with respect to other techniques.

Thoughts about sampling the visual world with better accuracy as compared with that of the existing techniques of the time arose as early as 1908 with Lippmann [48] and later Ives in 1928 [38] proposing the integral camera. This chapter reviews the stateof-the-art work in the fields of light field imaging right from its inception in 1908 to its commercialization in 2006 (Lytro camera) and beyond.

Integral photography proposed by Lippmann [48] was one of first techniques to capture the light field information. He proposed to use an array of lenslets (film protrusion that acts as converging lens) in front of a photographic film in order to record multiple images. Later Ives [38] in 1928 proposed a technique that used a grating in front of a film along with a main lens to record what he called a parallax panoramagram.

Binocular stereo systems [31] [69] were yet another approach that used two cameras to passively estimate the depth information. The depth was estimated by determining the correspondence between the images captured by the two cameras. The need for using two cameras made the setup bulky and it could estimate depth along only one axis owing to the parallax along that axis. The depth estimation error exponentially increased for increasing baseline distance between the cameras as a small triangulation error translated to a large distance estimation error. Further, failure of establishing 
proper correspondence in texture-less regions of images imposed a major limitation to the technique. Trinocular systems [37] make use of three cameras and offer better correspondence and depth estimates along multiple axis, but require complicated setup and processing algorithms to make them work robustly.

Bolles et al. [11] made use of motion parallax by moving a camera along a track and taking dense sequence of images. The technique made use of known camera position to approximate 3D information and spatiotemporal events (occlusion). However it could only capture static scenes and required precise control and movement of the gantry system.

Some of the computational photography techniques such as depth from focus (DFF) [57] [8] that were developed used a set of images (usually 10 to 12) captured by focusing the camera lens to different depths of the scene. This is an example of epsilon photography [63], where epsilon is the camera focus parameter. Pentland [61] later provided a solution in which only two images were required - one defocused and another focused at a particular depth. This technique was later expanded to a more general depth from defocus (DFD) solution [62] [75], wherein only two defocused images were required. The performance of this technique was further enhanced by capturing multiple defocused images. The main limitations of these techniques were the need for multiple images (making them useless for dynamic scenes) and low SNR of the reconstructed image when a small set of captured images were used for reconstruction.

Yet another class of techniques made use of an active light source for projecting patterns onto the scene of interest in order to gauge depth [28] [27] [58] [53]. These techniques were limited by the obvious need for an active source, which made them unsuitable under certain illumination conditions. They also consumed more power, which limited their use in mobile applications. Also, recovering the original image was a hassle as the overlaid pattern had to be removed by post processing. They also required prior calibration which was possible under all circumstances. 
In 1992 Adelson et al. [4] proposed a portable camera setup. It was bulky and used external relay lenses to focus the focal plane of the microlens array onto the image sensor. It further used a field lens to focus images beneath the microlens. It was the first major attempt in the modern era to bring light field imaging back to prominence.

Levoy et al. [46] in 1996 proposed a movable camera gantry that used a computer controlled gantry to capture dense images. They also introduced a rendering technique based on light slabs and demonstrated novel view synthesis from the captured data. Meanwhile, around the same time, Gortler et al. [30] used a hand-held camera to capture images of objects placed in a capture stage and used calibration markers to estimate camera's position and orientation.

A general version of multi-aperture camera was designed by Wilburn et al. [95] called the Stanford multi-camera array and used a number of inexpensive cameras that could generate video data up to 1 giga-samples per second. The cameras could be flexibly arranged and was used to demonstrate applications such as synthetic aperture videography, high speed videography, and spatiotemporal view interpolation. Vaish et al. [80] used this setup to demonstrate synthetic aperture photography.

In the following sections we describe in detail the techniques of plenoptic imaging and Talbot imaging as they will help us to critically analyze the techniques developed in this thesis.

\subsection{Plenoptic Imaging}

A plenoptic (multi-aperture) image sensor uses two lenses for forming image onto a sensor (figure 3.1). The first lens, similar to a conventional camera is the main lens with a big aperture. The second lens, called the microlens, is a set of small lenses placed at the focal plane of the first lens [60]. This ensures that the main lens is fixed at the microlens' optical infinity as the microlens is very small compared to the main lens. Further, to 
ensure maximum utilization of the image sensor pixels the main lens and the microlens have the same f number. Each microlens has a set of pixels underneath it. The number of microlenses in a sensor determines its spatial resolution and the number of pixels underneath each microlens determines its directional (angular) resolution.

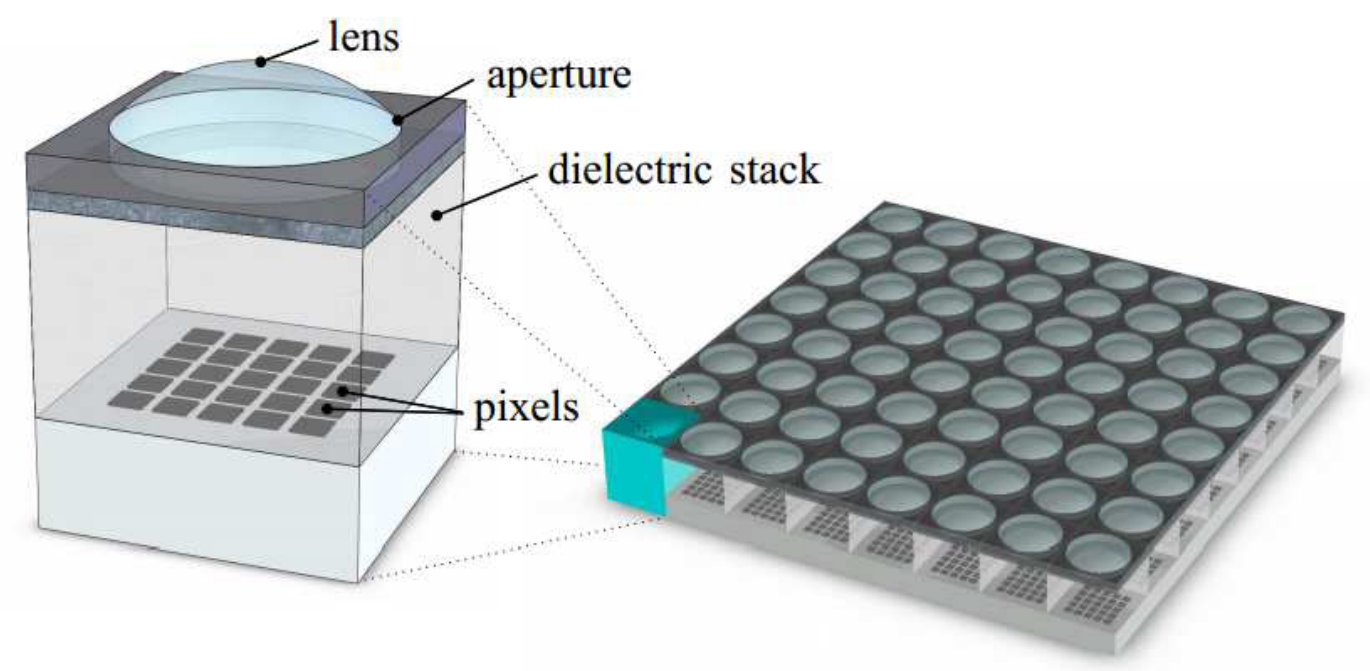

Figure 3.1: Microlens array in a multi-aperture image sensor [17].

The image formation process in plenoptic imaging is shown in figure 3.2. The object is focused onto the microlens plane which sorts the rays it receives onto pixels underneath it. Light that is recorded by a particular sensor pixel passes through its parent microlens and comes from a particular part of the main lens. In order to reconstruct an image as viewed from that particular sub-aperture of the main lens (figure 3.3), we have to integrate light from the shifted pixels underneath each microlens. The amount of shift is determined by the chosen main lens sub-aperture.

Figure 3.4 shows two sub-aperture images reconstructed by choosing the highlighted pixels in the figure. The two sub-apertures are from the opposite ends of the main lens and consequently the reconstructed image exhibits vertical parallax. 


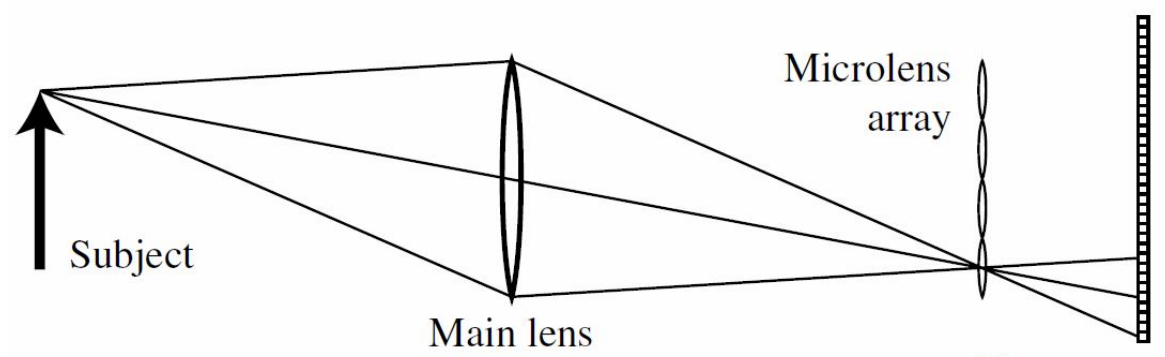

Photosensor

Figure 3.2: Principle of plenoptic imaging [60].
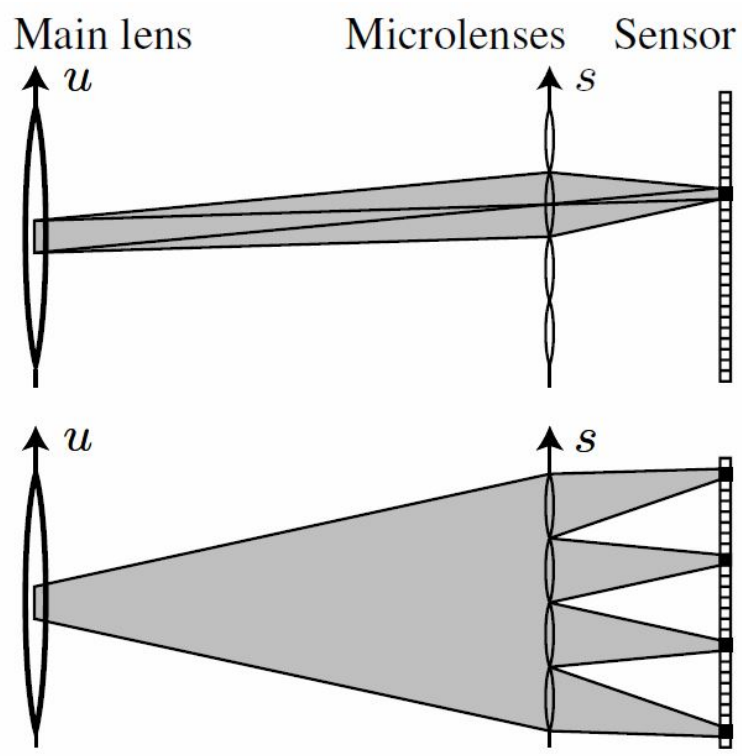

Figure 3.3: Sub-aperture image in plenoptic imaging [60].

\subsection{Focused Plenoptic Imaging}

The focused plenoptic imaging technique was developed to overcome the low spatial resolution that resulted from the plenoptic imaging technique. This technique trades the angular resolution for spatial resolution. The lost angular resolution can be interpolated, for example, by using techniques such as three-view morphing [24]. Like plenoptic imaging the focused plenoptic technique uses two sets of lenses, but instead of focusing the main lens image onto the microlens plane, it focuses slightly above the microlens plane as shown in figure 3.5. Also, unlike the plenoptic camera, the sensor is placed at a distance 

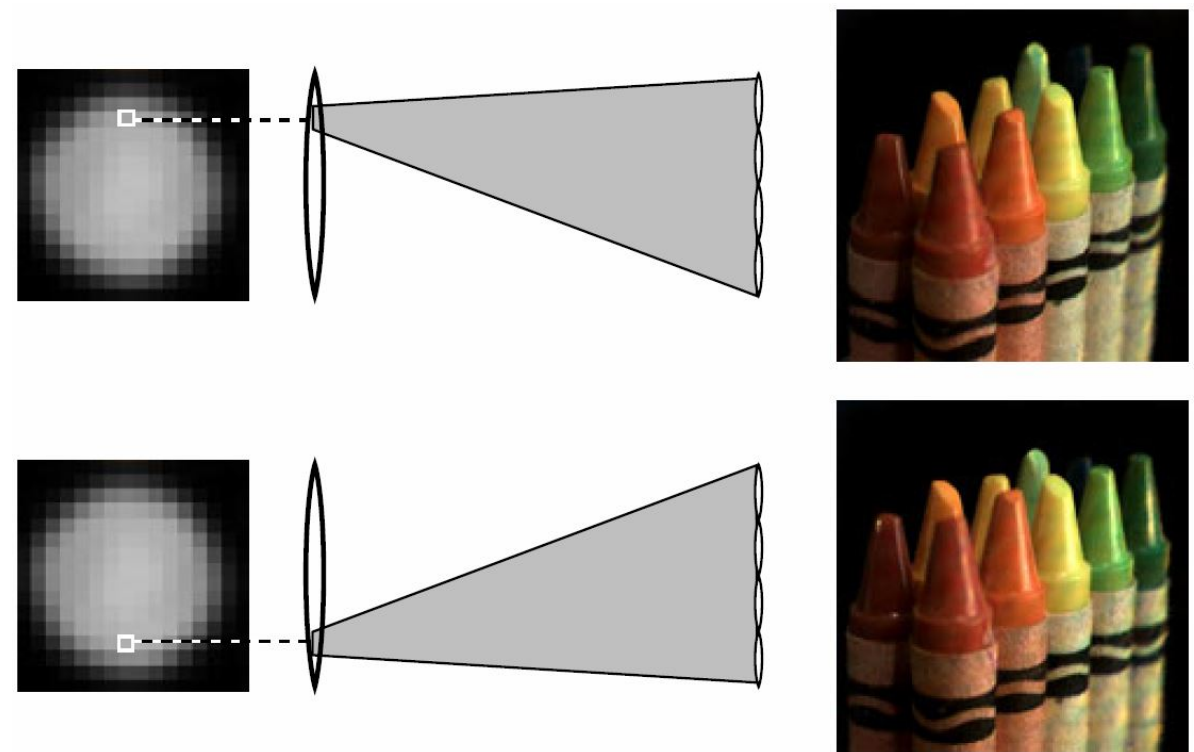

Figure 3.4: Two sub-aperture images formed by collecting highlighted pixels from the same position underneath each microlens [60].

$\mathrm{b}$, which is greater than $\mathrm{f}$, the lens focal length.

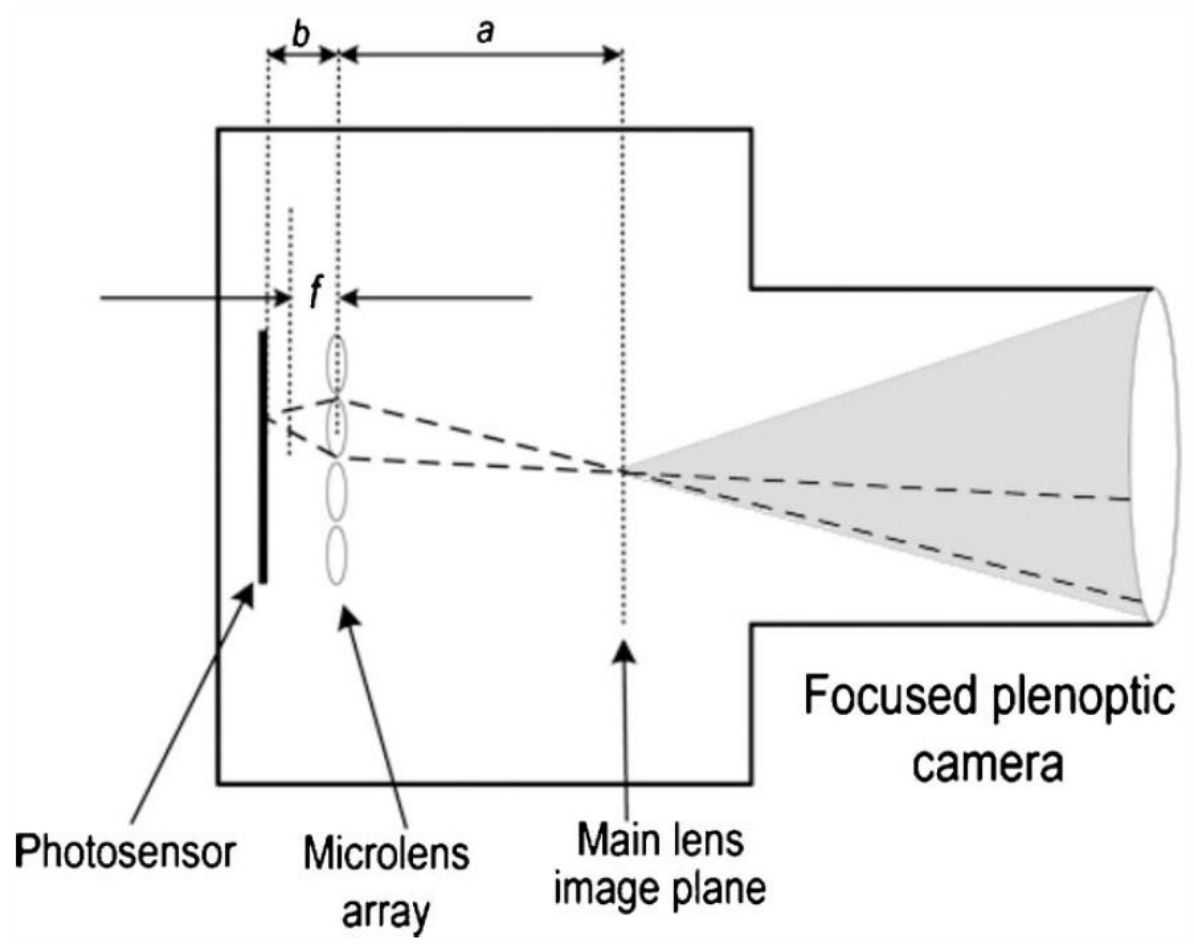

Figure 3.5: Image formation process in a focused plenoptic camera (figure from [25]). 
Unlike the plenoptic camera where the spatial resolution is fixed (dependent on the number of microlenses), in focused plenoptic camera the spatial resolution is dependent on the ratio a/b, the minification of the image. The increase in resolution is b/a. Also, unlike plenoptic camera, which integrates pixel information underneath a single microlens, the focused plenoptic camera integrates pixel information across multiple microlenses.

Both the plenoptic and focused plenoptic cameras use microlenses for light field capture and enforce a rather rigid trade-off between spatial and angular resolution (although it is slightly relaxed in the focused plenoptic camera). Even when an image is in perfect focus, there is still some loss of spatial resolution. This is overcome by the new class of techniques, christened angle sensitive imaging. In angle sensitive imaging, each angle sensitive pixel directly captures the local incident angle information. This enables sensors designed using angle sensitive pixels to have very high spatial resolution when the image is in focus, with the resolution degrading in proportion to the amount of defocus.

\subsection{Talbot Imaging}

Talbot imaging makes use of diffraction based Talbot effect to determine the incident light angle. Talbot effect [76] relates to the self imaging property of periodic diffraction gratings. The self images are a result of Fresnel diffraction and their location as determined by Rayleigh [65] is given by Eq. (Eq. 3.1), where $\mathrm{d}$ is the grating pitch and $\lambda$ is the wavelength. When $\lambda /$ d is small, Eq. (Eq. 3.1) reduces to Eq. (Eq. 6.1), which is the well know Talbot depth. Strong intensity patterns occur at depths that are half-integer multiples of the Talbot depth. The Talbot response is sensitive to the angle of incident light. This effect, known as the off-axis Talbot effect [77], forms the basis of Talbot effect based pixels.

$$
\mathrm{z}=\frac{\lambda}{1-\sqrt{1-\lambda^{2} / \mathrm{d}^{2}}}
$$




$$
\mathrm{z}_{\mathrm{t}}=\frac{2 \mathrm{~d}^{2}}{\lambda}
$$

The pioneering work on angle detection, which was based on Talbot effect [88], employs two levels of diffraction gratings. The second grating is placed at a depth 'z', below the first one and is known as the analyzer grating. It is placed to either block or pass the incident light and acts as a mask. The Talbot pixels are divided into groups, with each group having 4 pixels, each with distinct offset for the secondary grating. With 'D' as the grating pitch, the secondary gratings have offsets of ' 0 ', ' $\mathrm{D} / 2$ ', ' $\mathrm{D} / 4$ ' and ' $3 \mathrm{D} / 4$ '. The pixels with secondary grating offsets of ' 0 ' and 'D/2' work as a pair and those with grating offsets 'D/4' and '3D/4' work as a pair. Fig. 3.6.a illustrates this grating configuration. Fig. 3.6.b shows the formation of self-images at multiples of Talot depth, $\mathrm{z}_{t}$. The pixel responses are paired to eliminate ambiguity that arises when secondary grating with a particular offset blocks bright light at a certain angle, but passes dim light at another angle. This ineffectiveness of the Talbot pixel with only a single secondary grating without any offsets lead to incorrect results. In order to resolve this ambiguity, pixels with complementary secondary grating offsets are made to work as pairs [88].

The response produced by a Talbot pixel can be described by the below equation (Eq. 3.3).

$$
\mathrm{I}=\mathrm{I}_{0}(1+m \cos (\beta \theta+\alpha))
$$

$\mathrm{I}_{0}$ is the incident light intensity, $\mathrm{m}$ is the modulation depth which gives an indication about the strength of the angle dependent response, $\beta$ is the angular sensitivity, which dictates the periodicity of the response and its sensitivity to changes in incident angle. Since this response depends on both the intensity and angle information, complementary pixel responses are required to uniquely determine the incident angle. Sample response produced by the Tablot pixels of figure 3.6 is shown in figure 3.7. The periodic nature 

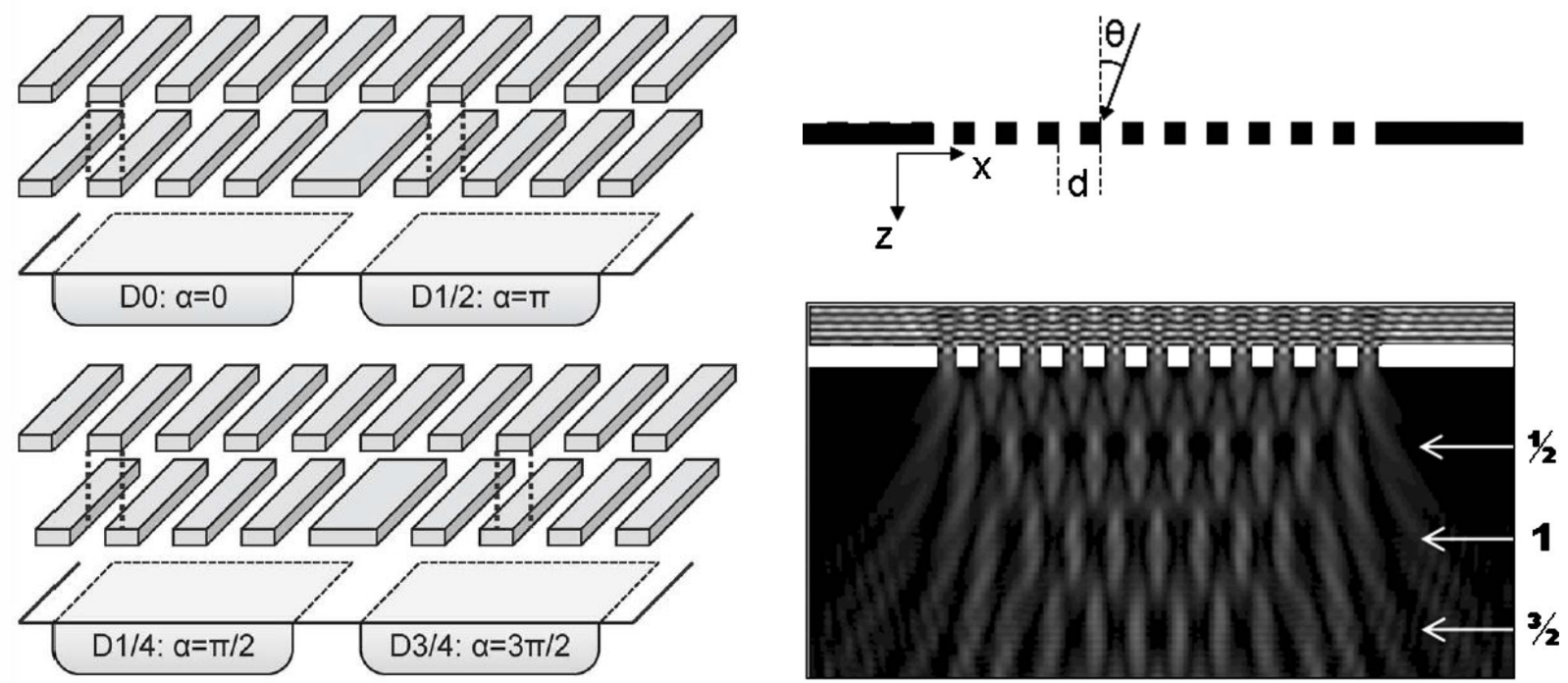

3.6.a: Talbot pixels with secondary grating offsets of $0, \mathrm{D} / 4, \mathrm{D} / 2$ and $3 \mathrm{D} / 4[88]$

3.6.b: Figure showing Talbot grating pitch and mulitples of Talbot depth, $\mathrm{z}_{t}[91]$

Figure 3.6: Figure showing the physical structure of Talbot pixels and self-image formation.

of the response imposes limits on the range of recoverable angles from a single complementary pair, necessitating multiple complementary pairs with varying periodic angle sensitivities.

Each Talbot pixel group is characterized by distinct directional gratings (horizontal, vertical or diagonal) and unique angle sensitivities. Talbot pixels can only produce response for light source variations that are orthogonal to the grating used. Thus, in order to detect variations in horizontal direction, we need a vertical grating and vice versa. Higher angle sensitivity means large variation in response for small change in angles, but with lower range of resolvable angles. On the other hand, lower angle sensitivity means small variation in response for large change in angles, but with higher range of resolvable angles. This is a design trade-off and cannot be eliminated. In order to overcome this limitation, earlier designs using Talbot effect based pixels used a number of pixel groups, each with distinct angle sensitivities.

One issue with Talbot pixel based design is the need to have pixel groups with different 

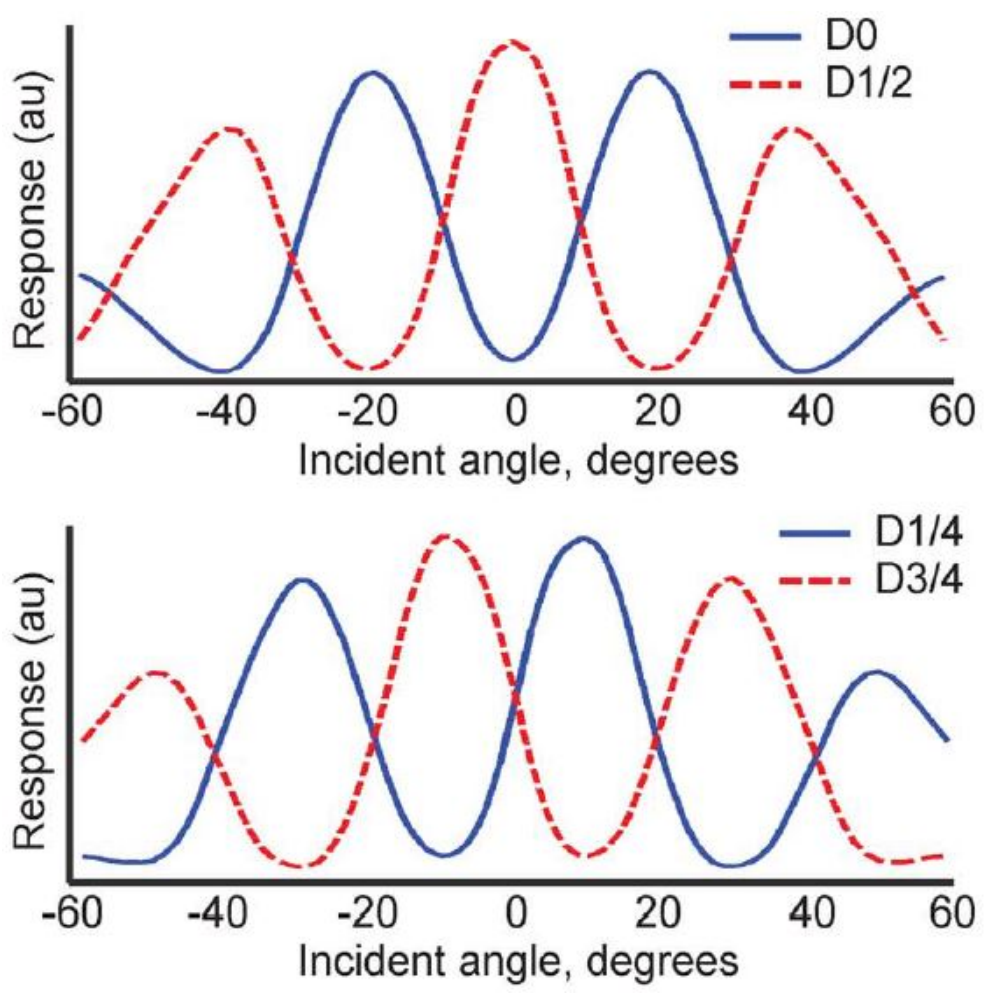

Figure 3.7: Sample response produced by Talbot pixels of figure 3.6 as a function of incidence angle variation [88].

angle sensitivity values for resolving a wide enough angle range. This leads to a number of redundant pixels in the sensor, which directly translates to a large area overhead. Another issue relates to the reduced light transmittance due to the presence of two layers of metal gratings on top of the photodiode.

A number of applications of angle sensitive imaging were demonstrated using the Talbot pixel sensor, namely, 3D imaging [86], lensless 3D imaging [85], post-capture image refocus [88], opto-electronic image compression [89] and optical flow sensing [87]. These serve as a motivation for exploring angle sensitive imaging. 


\subsection{Enhanced Talbot Imaging}

Enhanced Talbot pixels follow the same angle detection principle as the Talbot pixels, but use either one or no metal gratings on top of the pixels. In [72] and [71] four enhanced Talbot pixel designs were explored to increase the light transmittance and are briefly discussed here.

The conventional Talbot pixel uses a pair of metal (amplitude) gratings and uses an $\mathrm{N}$-well/P-sub diode to produce angle dependent behavior. By eliminating the secondary (analyzer) grating and using an Interleaved $\mathrm{N}+/ \mathrm{P}$-sub diode they report an improvement in light transmittance by a factor of 1.5 times the amplitude-amplitude grating Talbot sensor. Figure 3.8(a) shows the structure of the interleaved N+/P-sub diode.

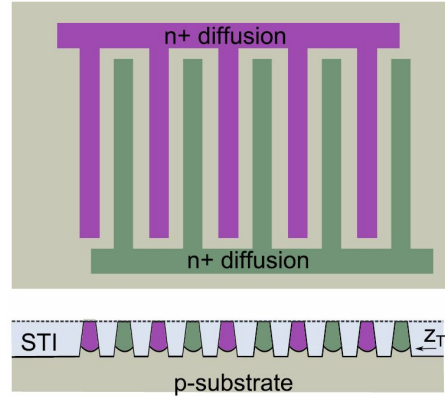

3.8.a: Interleaved $\mathrm{N}+/ \mathrm{P}-\mathrm{sub}$ diode

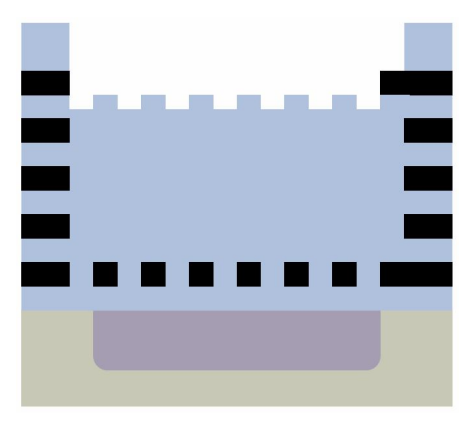

3.8.b: Phase grating with $\mathrm{N}-$ well/P-sub diode

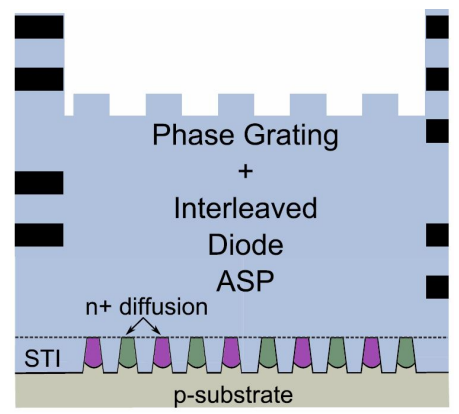

3.8.c: Phase grating with interleaved $\mathrm{N}+/ \mathrm{P}$-sub diode

Figure 3.8: Figure showing three of the enhaced Tablot pixels proposed in [72] (figures from $[72])$

Instead of the interleaved $\mathrm{N}+/ \mathrm{P}$-sub diode if one uses interleaved $\mathrm{P}+/ \mathrm{N}$-well diode placed inside an N-well, an improvement of 2.4 times the original is noted. This is the same improvement obtained if one replaces the primary amplitude grating with a phase grating (formed by etching the dielectric layer after device fabrication) and a single Nwell/P-sub diode with an amplitude secondary grating (figure 3.8(b)). An improvement on the order of 3.75 times is observed when one uses a phase grating with an interleaved $\mathrm{N}+/ \mathrm{P}$-sub diode (figure $3.8(\mathrm{c})$ ). 
If one uses enhanced Talbot pixels with phase gratings as opposed to metal gratings, post fabrication processes are required for manufacturing the sensor. Care must be taken to keep the environment free of micro particles and precautions must be taken to avoid over-etching (changes the modulation depth 'm') and improper mask alignment. This increases the complexity and cost for manufacturing such a device. On the other hand, the interleaved diodes are formed either with $\mathrm{N}+/ \mathrm{P}$-sub or $\mathrm{P}+/ \mathrm{N}$-well and have very shallow depletion widths. Owing to a shallow depletion width these interleaved diodes have lower sensitivity and SNR as opposed to the N-well/P-sub diodes [56]. 


\section{Chapter 4}

\section{Angle Sensitive Imaging using Metal Masks}

This chapter takes one through all the techniques that were experimented upon in order to devise an efficient method for light field capture. We introduce a new technique based on metal shading called the quadrature pixel cluster (QPC) and propose improvements to the base design in the later sections. We conclude the chapter with multi-finger (MF) angle detecting pixels that are based on the same technique as the QPC, but provide more robust angle detection capabilities.

\subsection{Quadrature Pixel Cluster}

Through this work we propose a new technique for angle detection. This technique, called the track-and-tune angle detection, makes use of two types of pixels for angle detection. The first type, called the Quadrature Pixel Cluster (QPC), is based on the concept of metal shading [41] [40] and produces linear response proportional to angle variations, but with low sensitivity. The second type, called the Talbot pixel [88], produces precise nonlinear response proportional to the angle variations. The track-and-tune technique makes use of the linear response of QPC for coarse angle detection and non-linear response of Talbot pixels for fine angle tuning. Hence, by using two different pixel types, the 
complexity of the angle detection process is greatly reduced, thereby decreasing the number of pixels needed for angle detection and increasing the sensor resolution.

A major advantage of the proposed method is that a single macro-pixel (comprising of 13 sub-pixels) is capable of determining the angle of a 3D point. This is in stark contrast to the Talbot effect based pixel design that used 32 pixels [88] (for only horizontal and vertical directions) for producing similar results. Moreover, the technique is independent of the wavelength of incident light and consequently can be applied more reliably to natural scenes that contain objects illuminated by light of different wavelengths.

\subsubsection{Theory}

Fig. 4.1 shows the perspective view of the proposed structure. It consists of four photodiodes (N-well/P-sub) with a metal block on top of it. The metal block is implemented in one of the layers of CMOS metal stack and partially covers the photodiodes. The area covered by the metal block is symmetrical along the $\mathrm{X}$ and $\mathrm{Y}$ directions for each of the four photodiodes. Each photodiode along with a set of transistors form the pixel.

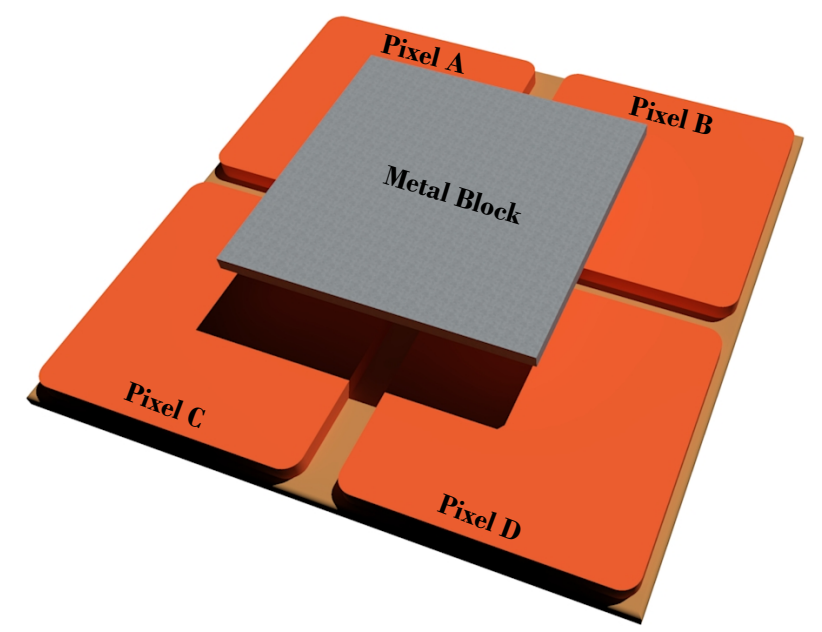

Figure 4.1: Physical structure of angle sensitive quadrature pixel cluster.

The metal layer is present in a complex assortment of inter metal dielectrics, thin ion migration barrier layers (TiN) and $\mathrm{SiO}_{2}$. For the sake of simplicity we consider all these 


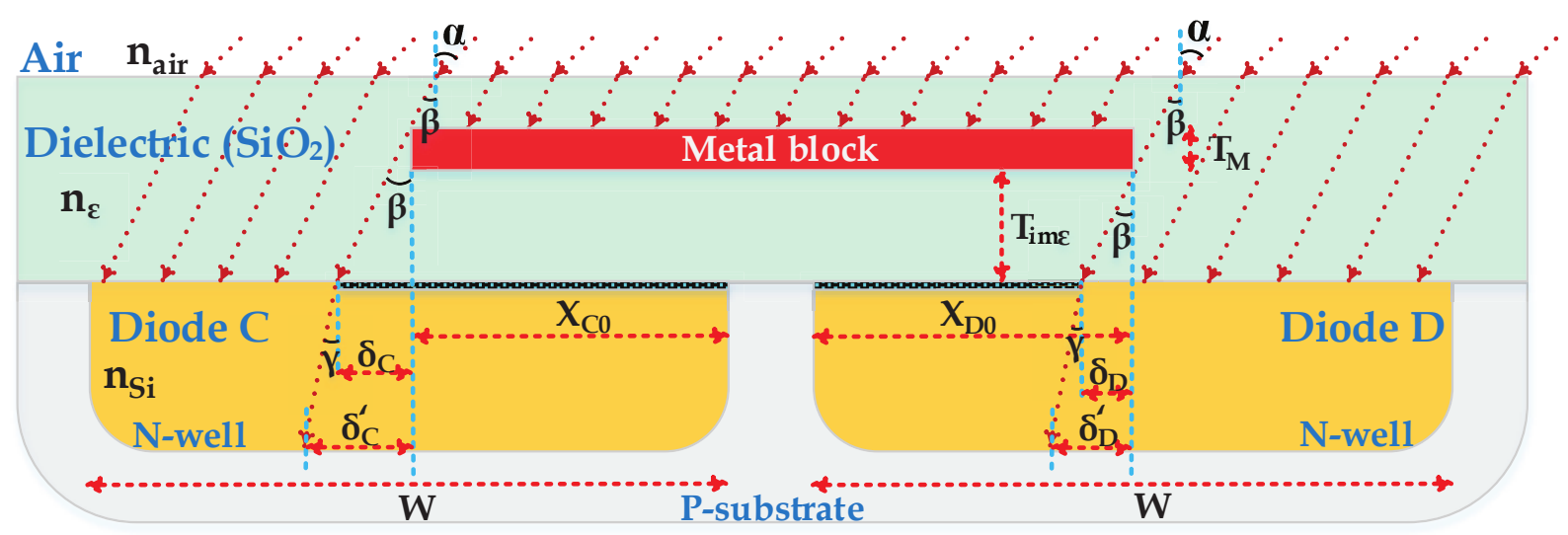

Figure 4.2: 2D view of quadrature pixel cluster along the $\mathrm{X}$ direction.

dielectrics as a single dielectric having the refractive index of $\mathrm{SiO}_{2}$.

Fig. 4.2 shows the two dimensional view of a QPC along the $\mathrm{X}$ direction. $\alpha$ is the incident light angle, $\beta$ is the transmitted light angle at the air $/ \mathrm{SiO}_{2}$ interface and $\gamma$ is the transmitted light angle at the $\mathrm{SiO}_{2} / \mathrm{Si}$ interface. The angle $\gamma$ is the one that is of interest to us, since it is this angle that determines the amount of light falling on the pn junction. However, $\gamma$ is difficult to determine because of the difficulty in estimating the depth and doping of the n-well layer (doping influences the refractive index), both of which are process parameters and hence confidential.

$\mathrm{W}$ is the width of the photodiode and since the diode is symmetrical, its area is given by Eq. (Eq. 4.1). $\mathrm{X}_{\mathrm{C} 0}$ and $\mathrm{X}_{\mathrm{D} 0}$ are the widths of the shaded regions of the diodes under normal illumination $\left(\alpha=0^{\circ}\right)$ and the shaded areas are given by Eq. (Eq. 4.2) and Eq. (Eq. 4.3) respectively.

$$
\begin{gathered}
\mathrm{A}=\mathrm{W} \times \mathrm{W} \\
\mathrm{A}_{\mathrm{shC}}=\mathrm{X}_{\mathrm{C} 0} \times \mathrm{X}_{\mathrm{C} 0} \\
\mathrm{~A}_{\mathrm{shD}}=\mathrm{X}_{\mathrm{D} 0} \times \mathrm{X}_{\mathrm{D} 0}
\end{gathered}
$$


$\delta_{\mathrm{C}}$ and $\delta_{\mathrm{D}}$ are the change in the shading widths at the $\mathrm{SiO}_{2} / \mathrm{Si}$ interface for nonnormal (other that $0^{\circ}$ ) incidence of light. $\delta_{\mathrm{C}}^{\prime}$ and $\delta_{\mathrm{D}}^{\prime}$ are the actual shading widths at the pn junction that influence the magnitude of the photocurrent in each of the two diodes, $\mathrm{C}$ and D. Since $\gamma$ cannot be accurately determined, the actual metal shading widths $\delta_{\mathrm{C}}^{\prime}$ and $\delta_{\mathrm{D}}^{\prime}$ also cannot be accurately determined. Hence $\delta_{\mathrm{C}}^{\prime}$ and $\delta_{\mathrm{D}}^{\prime}$ are approximated by $\delta_{\mathrm{C}}$ and $\delta_{\mathrm{D}}$, which can be determined fairly accurately because $\beta$ can be accurately determined. This approximation is valid because the depth of the n-well is very small compared to the dielectric stack and hence the error is very small.

Let $\mathrm{n}_{\text {air }}$ and $\mathrm{n}_{\varepsilon}$ be the refractive indices of air and dielectric respectively. Then, from Snell's law [33] we can write the transmitted angle $\beta$ as

$$
\beta=\operatorname{arcSin}\left(\frac{\mathrm{n}_{\text {air }}}{\mathrm{n}_{\varepsilon}} \operatorname{Sin} \alpha\right)
$$

Let $\mathrm{T}_{\mathrm{im} \varepsilon}$ be the thickness of the inter metal dielectric stack from the bottom of the $\mathrm{M}^{\text {th }}$ metal layer downwards and let $\mathrm{T}_{M}$ be the thickness of the $\mathrm{M}^{\text {th }}$ metal layer, then the change in the shaded region, $\delta_{\mathrm{C}}$, of diode $\mathrm{C}$ is given by

$$
\delta_{\mathrm{C}}=\left(\mathrm{T}_{\mathrm{im} \varepsilon}+\mathrm{T}_{\mathrm{M}}\right) \tan \beta
$$

Similarly, the change in the shaded region, $\delta_{D}$, of diode $\mathrm{D}$ is given by

$$
\delta_{\mathrm{D}}=\left(\mathrm{T}_{\mathrm{im} \varepsilon}\right) \tan \beta
$$

Combining Eq. (Eq. 4.4) and Eq. (Eq. 4.5), we can write

$$
\delta_{\mathrm{C}}=\left(\mathrm{T}_{\mathrm{im} \varepsilon}+\mathrm{T}_{\mathrm{M}}\right) \tan \left(\operatorname{arcSin}\left(\frac{\mathrm{n}_{\text {air }}}{\mathrm{n}_{\varepsilon}} \operatorname{Sin} \alpha\right)\right)
$$

Similarly, by combining Eq. (Eq. 4.4) and Eq. (Eq. 4.6), we can write

$$
\delta_{\mathrm{D}}=\left(\mathrm{T}_{\mathrm{im} \varepsilon}\right) \tan \left(\operatorname{arcSin}\left(\frac{\mathrm{n}_{\mathrm{air}}}{\mathrm{n}_{\varepsilon}} \operatorname{Sin} \alpha\right)\right)
$$


Eq. (Eq. 4.7) and Eq. (Eq. 4.8) can be used to model a QPC and they help to determine the appropriate value of $\mathrm{T}_{\mathrm{im} \varepsilon}$ and $\mathrm{T}_{\mathrm{M}}$ for an acceptable response. $\mathrm{T}_{\mathrm{im} \varepsilon}$ and $\mathrm{T}_{\mathrm{M}}$ can take only a distinct set of values and are dependent on the CMOS process.

When the light angle is positive $\left(0^{\circ}<\alpha \leq 90^{\circ}\right)$ and varies along the horizontal $(\mathrm{X})$ direction (with no variation along the vertical ( $\mathrm{Y}$ ) direction), diode $\mathrm{C}$ is shaded more than diode D (as shown in Fig. 4.2) and hence light intensity recorded by diode $\mathrm{C}$ will be less than that recorded by diode $\mathrm{D}$. The area of the shaded regions in each diode can be written as:

$$
\begin{aligned}
& \mathrm{A}_{\mathrm{shC}}=\mathrm{X}_{\mathrm{C} 0} \times\left(\mathrm{X}_{\mathrm{C} 0}+\delta_{\mathrm{C}}\right) \\
& \mathrm{A}_{\mathrm{shD}}=\mathrm{X}_{\mathrm{D} 0} \times\left(\mathrm{X}_{\mathrm{D} 0}-\delta_{\mathrm{D}}\right)
\end{aligned}
$$

Since A is the total diode area, the area of the unshaded regions can be obtained from the following equations:

$$
\begin{aligned}
& \mathrm{A}_{\mathrm{ushC}}=\mathrm{A}-\mathrm{A}_{\mathrm{shC}} \\
& \mathrm{A}_{\mathrm{ushD}}=\mathrm{A}-\mathrm{A}_{\mathrm{shD}}
\end{aligned}
$$

Since $A_{s h D}$ is less than $A_{s h C}, A_{u s h D}$ is greater than $A_{u s h C}$ and as a consequence diode $\mathrm{D}$ registers more response than diode $\mathrm{C}$. For negative angles $\left(-90^{\circ} \leq \alpha<0^{\circ}\right), \mathrm{A}_{\mathrm{ushD}}$ will be lesser than $\mathrm{A}_{\text {ushC }}$ and hence diode $\mathrm{C}$ will register more response than diode $\mathrm{D}$. As angle is varied from normal incidence to the maximum $\left(\alpha= \pm 90^{\circ}\right)$, the difference in the response produced by the diodes keep on increasing and reaches a limiting value at $\alpha= \pm 90^{\circ}$. Since this difference is linear, the angle information can be decoded easily.

If the light angle variation is along the vertical direction (with no variation along the horizontal direction), the difference in responses of the diode pair B and D (or A and C) will help us to determine the angles. 


\subsubsection{Simulations}

In order to verify correctness of the theoretical formulation constructed earlier, we performed simulations based on the derived equations. The simulations were performed by considering pixels with an area of $6 \times 6 \mu \mathrm{m}^{2}$, with the parameters Tim $=1.883 \mu \mathrm{m}$ and $\mathrm{Tm}=0.222 \mu \mathrm{m}$. The result is plotted in Fig. 4.3 and shows the relationship between difference response produced by unshaded areas $\left(A_{u s h C}-A_{u s h D}\right)$ of photodiodes as a function of incident light angle. The difference in the unshaded area has a direct correlation with the amount of intensity captured by the photodiodes. As the response produced by the photodiodes are dependent on the intensity of light falling on them, the difference in their response will be linear since the difference in their unshaded area is linear.

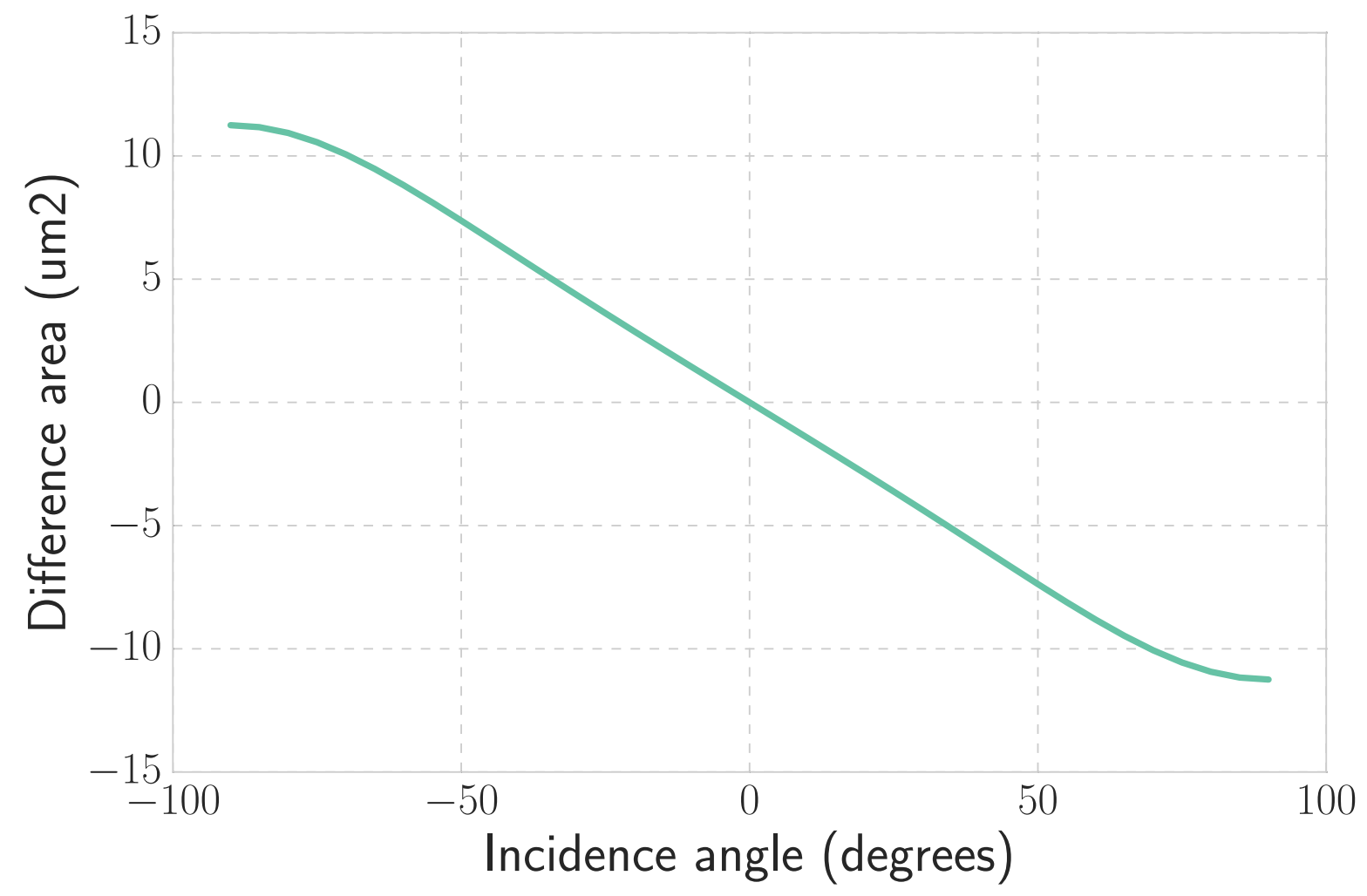

Figure 4.3: Difference in unshaded area as a function of incident angle for two adjacent photodiodes in a quadrature pixel cluster. 
FDTD simulations further consolidate the concept and help to determine the parameters that lead to an appreciable output response. The light angle was varied from $-45^{\circ}$ to $+45^{\circ}$ along the horizontal direction and diode responses were recorded. Fig. 4.4 shows the Electric field profile for negative $\left(-40^{\circ}\right)$ and positive $\left(+40^{\circ}\right)$ angles. The difference response is plotted in Fig. 4.5 and it shows a linear variation in recorded intensity as the angle is varied.

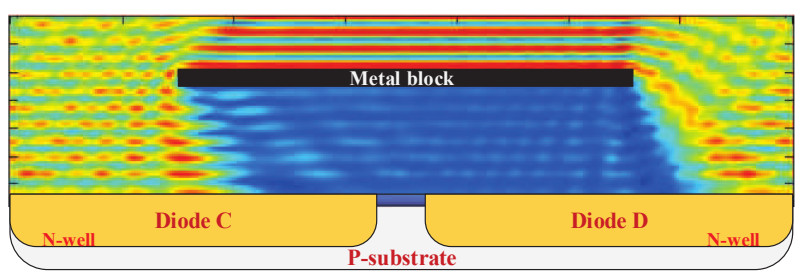

4.4.a: Negative angle $\left(\alpha=-40^{\circ}\right)$

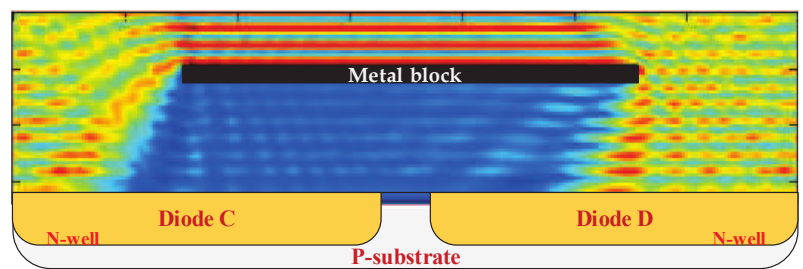

4.4.b: Positive angle $\left(\alpha=+40^{\circ}\right)$

Figure 4.4: FDTD simulations showing electric field profiles for a pair of pixels in a quadrature pixel cluster.

Although the response produced by QPC is linear, its sensitivity to angle variation is low. Angular sensitivity is defined as the strength of response for small changes in angle. If the strength of response is large for a small change in angle, the angular sensitivity is high. This means that the QPC can unambiguously resolve angles that are far apart, but not the ones that are close together.

Comparing Eq. (Eq. 4.7) and Eq. (Eq. 4.8) we see that the parameter $\mathrm{T}_{\mathrm{M}}$ is responsible for angular sensitivity. Larger the value of $\mathrm{T}_{\mathrm{M}}$, greater is the difference in the response produced by adjacent photodiodes. Fig. 4.6 shows the FDTD simulation results for different metal thicknesses. 1x refers to the thickness of lower metal layers (typically metal 1 to metal 5 or metal 6 depending on the process) in a CMOS metal stack, $4 \mathrm{x}$ and $12 \mathrm{x}$ refers to four times and twelve times the thickness of the lower level metal layers. These metal layers constitute the upper level metal stack of the CMOS process. The number of metal layers and the thickness of the layers vary from process to process. As 


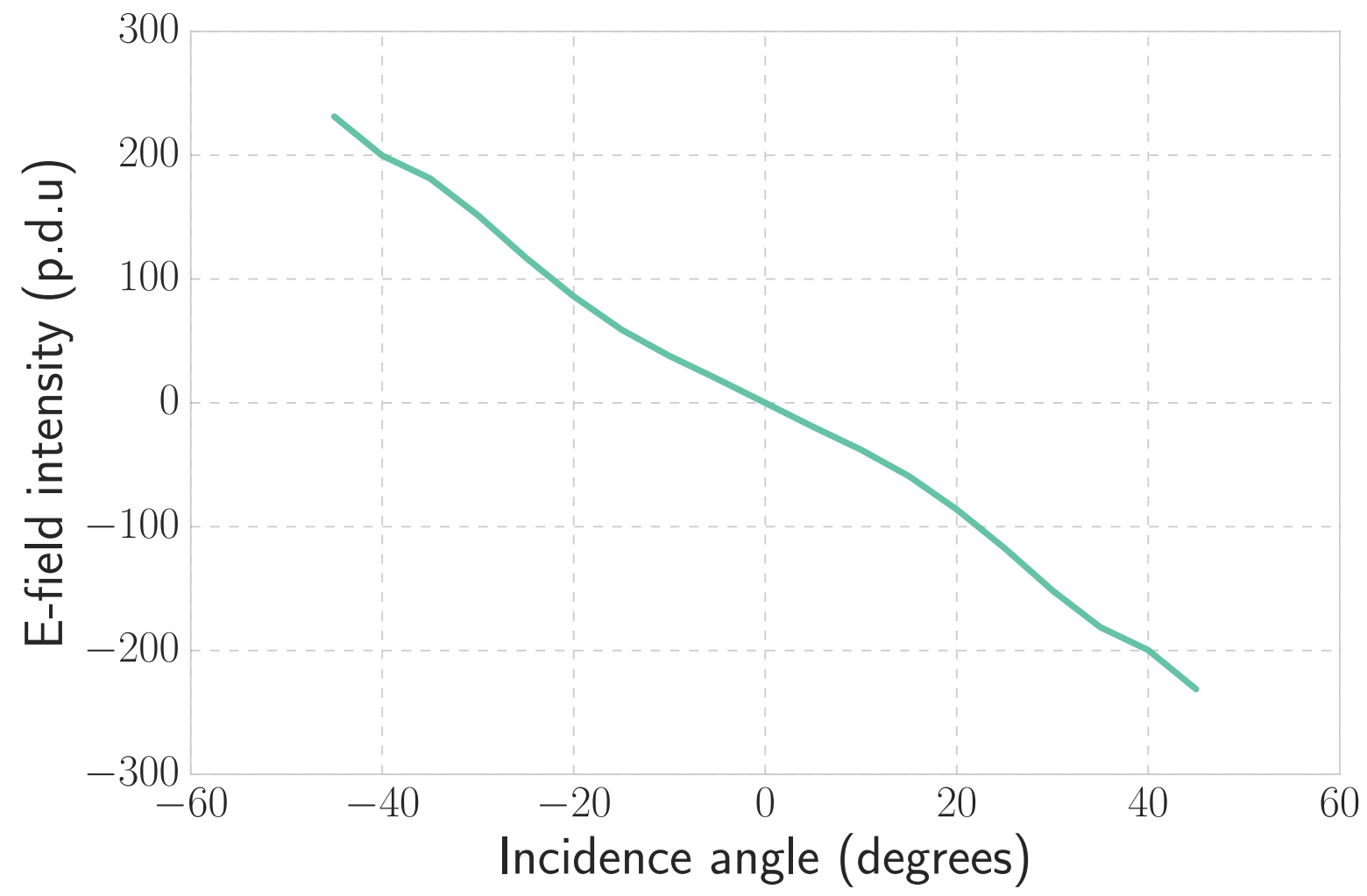

Figure 4.5: Variation in intensity as a function of incident angle for two adjacent photodiodes in a quadrature pixel cluster.

can be seen from the figure, as the thickness of the metal layer increases, the slope of the response (sensitivity) increases. Consequently, using a higher level metal layer leads to a more sensitive QPC and hence better angle resolution. Describing the sensitivity change quantitatively we find that the per degree change in response for metal of thickness $1 \mathrm{x}$, $4 \mathrm{x}$ and $12 \mathrm{x}$ are $2.713,4.373$ and 7.438 respectively.

In order to determine the effect of metal shading on the sensitivity of the response, we performed FDTD simulations by varying the amount of metal area that covers the photodiodes. Metal area over the photodiode was varied from $30 \%$ to $70 \%$ and the resulting values were plotted (Fig. 4.7). As can be seen, larger the area covered by metal over the photodiode, higher is the sensitivity. A quantitative measure of sensitivity (response per degree) for metal widths of $30 \%, 50 \%$ and $70 \%$ are $3.404,7.678$ and 7.438 


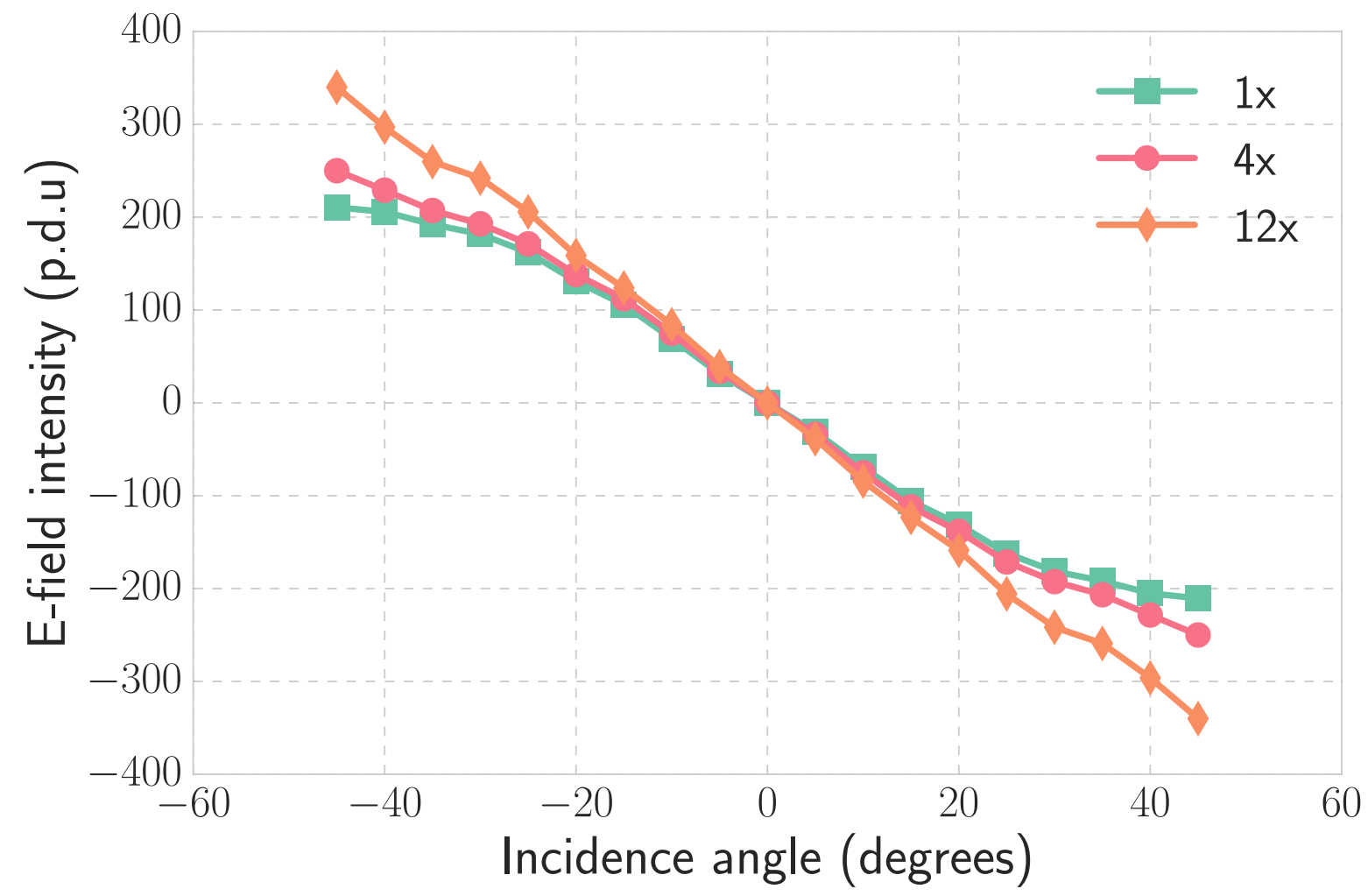

Figure 4.6: Quadrature pixel response as a function of incident angle for varying metal thickness.

respectively.

We had introduced the angle sensitive Talbot pixels in the previous chapter. Here we show some FDTD simulations to illustrate their behavior.

For simulations a pitch of $0.76 \mu \mathrm{m}$ with a duty cycle of $50 \%$ was used for both primary and secondary gratings. The secondary grating was placed at a depth where strong intensity patterns occurred corresponding to an effective wavelength of $373 \mathrm{~nm}(532 \mathrm{~nm}$ in vacuum). Fig. 4.8 shows the Finite Difference Time Domain (FDTD) simulation results for difference response produced by a pixel pair (either with $\alpha=0$ and $\alpha=\pi$ or with $\alpha=\pi / 2$ and $\alpha=3 \pi / 2$ ), for plane wave illumination, as source angle is varied from $-45^{\circ}$ to $+45^{\circ}$. As expected, the response is cosine in nature. 


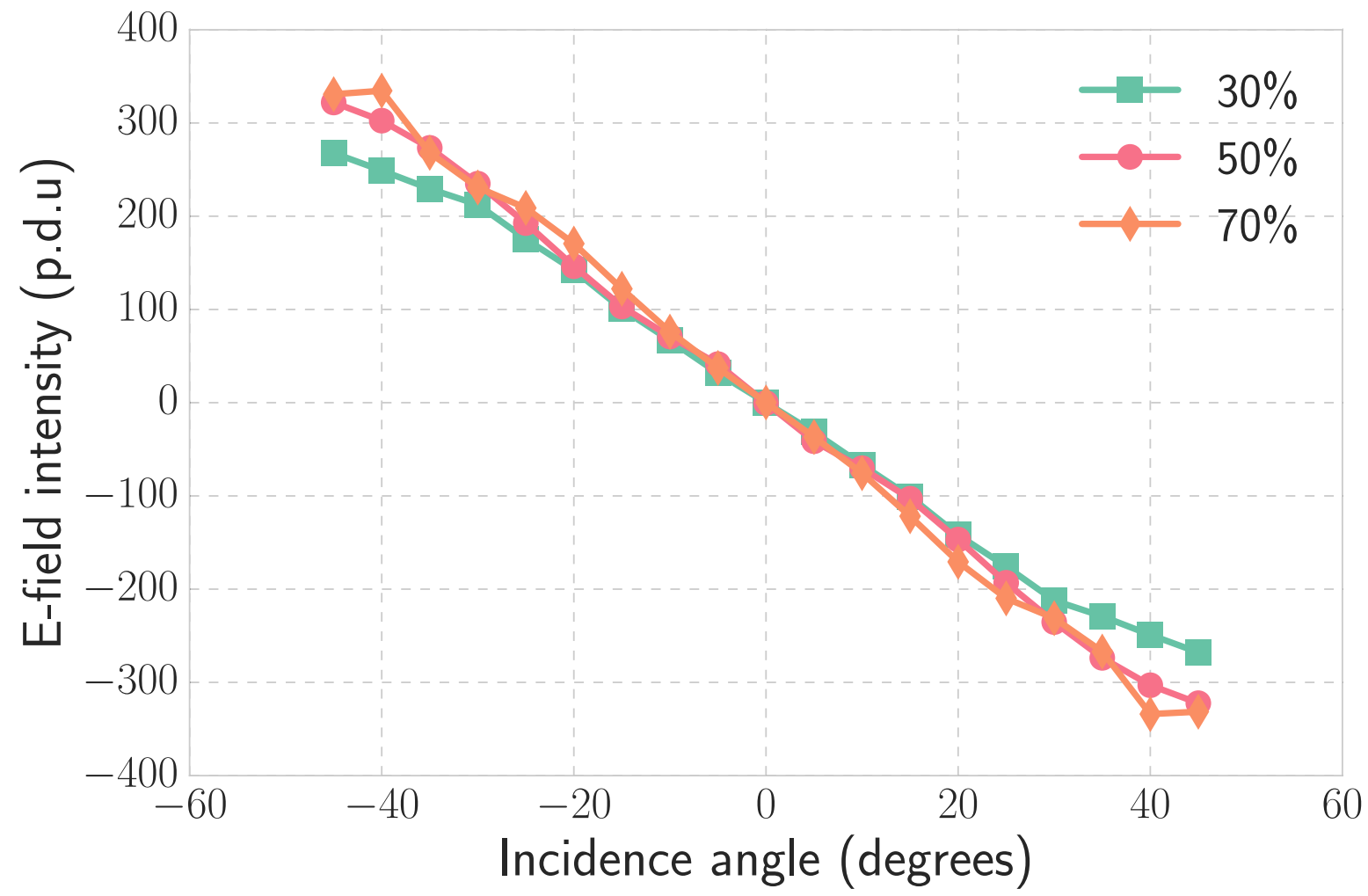

Figure 4.7: Quadrature pixel response as a function of incident angle for varying metal widths.

\subsection{Track-and-Tune Angle Detection Technique}

The two angle detecting pixel structures seen earlier (Talbot pixels and QPC) have their own merits and demerits. Talbot pixels have good sensitivity for small changes in angle, but are highly dependent on the wavelength, grating pitch and number of gratings on top of the photodiode. Furthermore, angle resolution needs pixels with different angle sensitivities and their directional dependence necessitates need for different directional gratings (horizontal, vertical and diagonal). QPC, on the other hand, is unaffected by the numerous factors that plague Talbot pixels, but have low angular sensitivity. A compromise is achieved by combining the positives that both the pixel structures have to offer. 


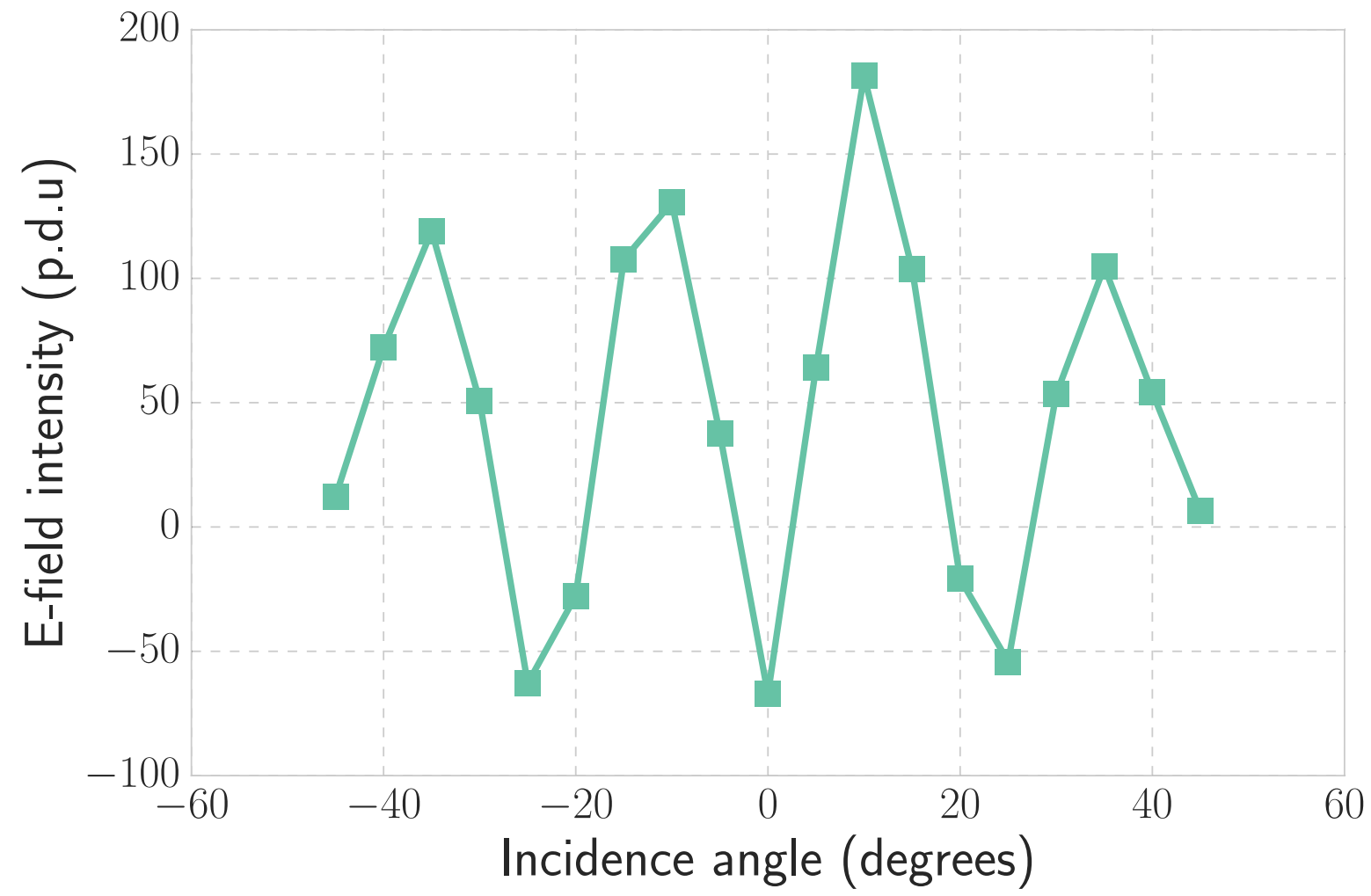

Figure 4.8: FDTD simulation of difference response produced by Talbot pixels $(\lambda=400$ $\mathrm{nm})$.

The track-and-tune angle detection technique [83] makes use of QPC to provide coarse estimate of angle and Talbot pixels for fine angle resolution. The principle is depicted in Fig. 4.9. Say, suppose the angle of incident light is $15^{\circ}$, the QPC will coarsely identify that the angle is around $15^{\circ}$, but will be unable to pin point the correct value. Now, once we know where the angle is located, we can use that particular segment $\left(\mathrm{S}_{1}-\mathrm{S}_{2}\right)$ of the periodic cosine Talbot pixel response to pin point the angle. Hence, this technique only requires a Talbot group with angular sensitivity pixels of a single kind and a single QPC for accurately determining the incidence angles. Since QPC can detect vertical and horizontal angles, a single QPC can be shared with a vertical (having vertical grating) and a horizontal (having horizontal grating) Talbot group. 


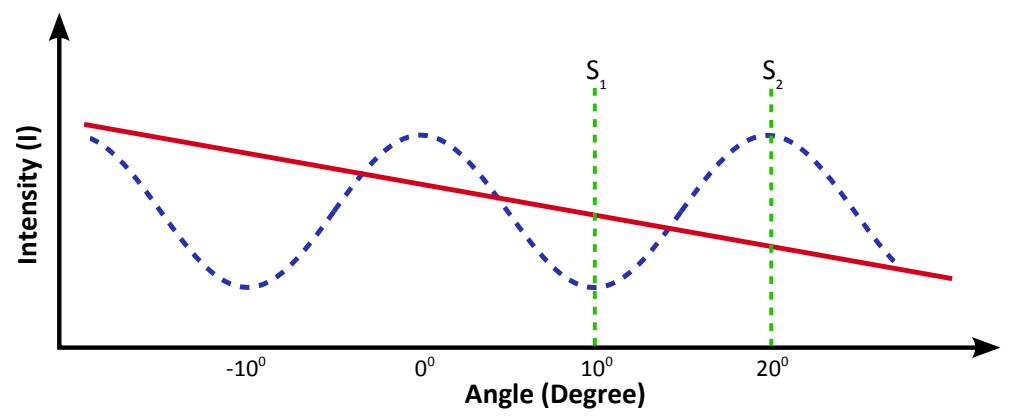

Figure 4.9: Technique illustrating angle detection using Talbot and QPC pixel responses.

\subsubsection{Sensor Architecture}

The proof-of-concept sensor occupies an area of $1.6 \mathrm{~mm} \times 1 \mathrm{~mm}$ and was fabricated in $65 \mathrm{~nm}$ GlobalFoundries mixed-signal CMOS process. Fig. 4.10 shows the sensor architecture along with the chip microphotograph. The pixel array is made up of $12 \times 10$ macro pixel clusters. Figure 4.11 shows the internal architecture of a macro pixel cluster. Each macro pixel cluster consists of 4 macro pixels, which share a switched capacitor amplifier. Each macro pixel, in turn, is made up of 13 distinct pixel types as shown in the figure. Pixels 1 to 8 are Talbot pixels and pixels 9 to 12 are QPC pixels. Pixel 13 is an ordinary pixel which captures only intensity. Table 4.1 lists the different pixel types along with the metal configuration used on top of the photodiodes. The pixels have been configured to produce difference response. Pixels 1, 2 and 3, 4 produce Talbot difference response for vertical angle variations (because of horizontal grating). Pixels 5, 6 and 7, 8 produce Talbot difference response for horizontal angle variations (because of vertical grating). Pixels 9, 10 and 11, 12 produce linear difference response for horizontal angle variations. Finally, pixels 9, 11 and 10, 12 produce linear difference response for vertical angle variations. Fig. 4.11 also shows ideal pixel responses.

The switched capacitor amplifier produces difference response and it amplifies the same by a factor of 2 . It is made up of a $7 \mathrm{~T}$ OpAmp and consists of two switches at the input. The input switches are made up of transmission gates (TG) and contain 

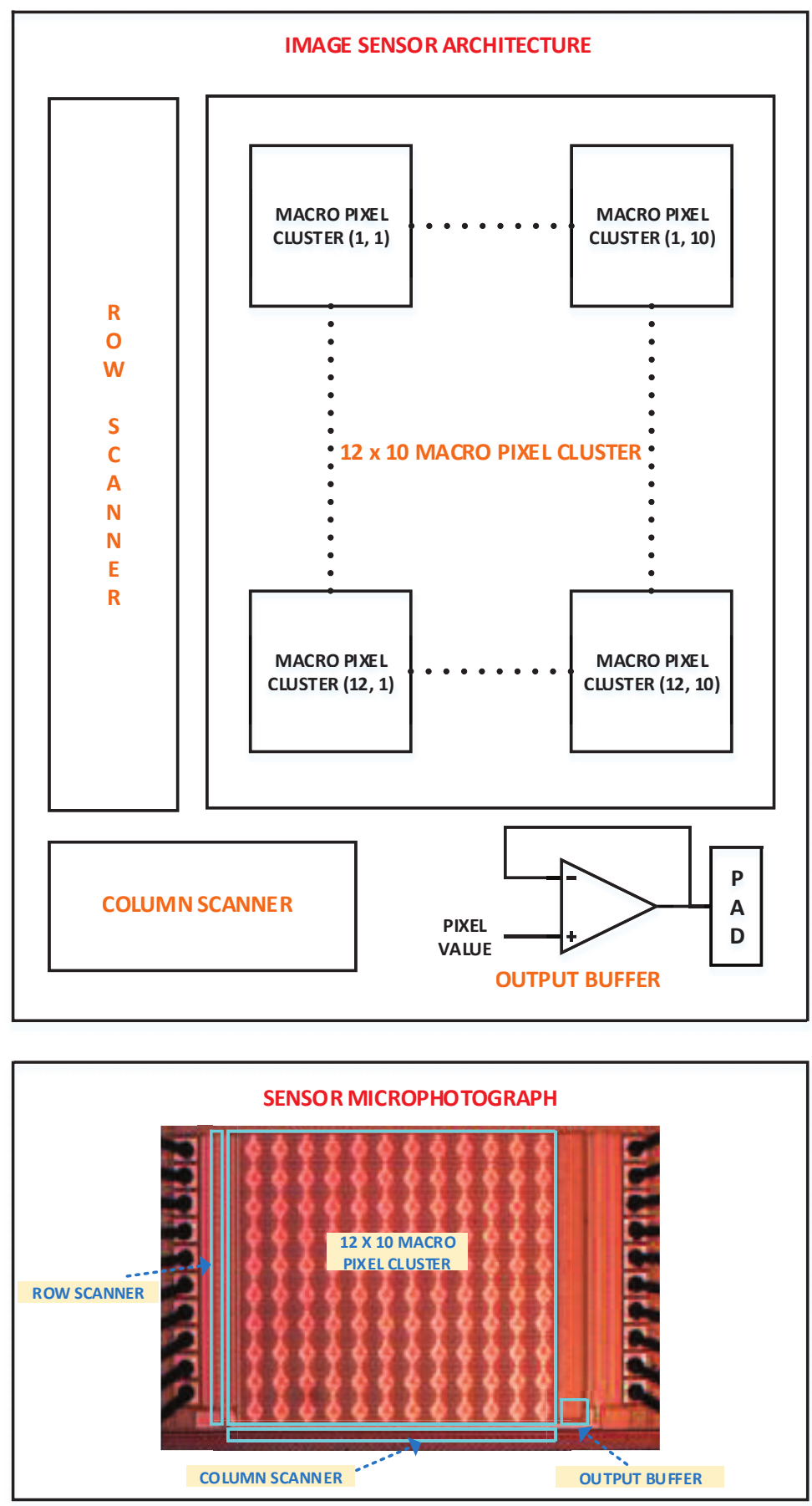

Figure 4.10: Sensor architecture along with chip microphotograph.

additional dummy transistors for cancelling the charge injection (CIC) into the input capacitor when TG is turned off. It produces output in accordance with Eq. (Eq. 4.13). 


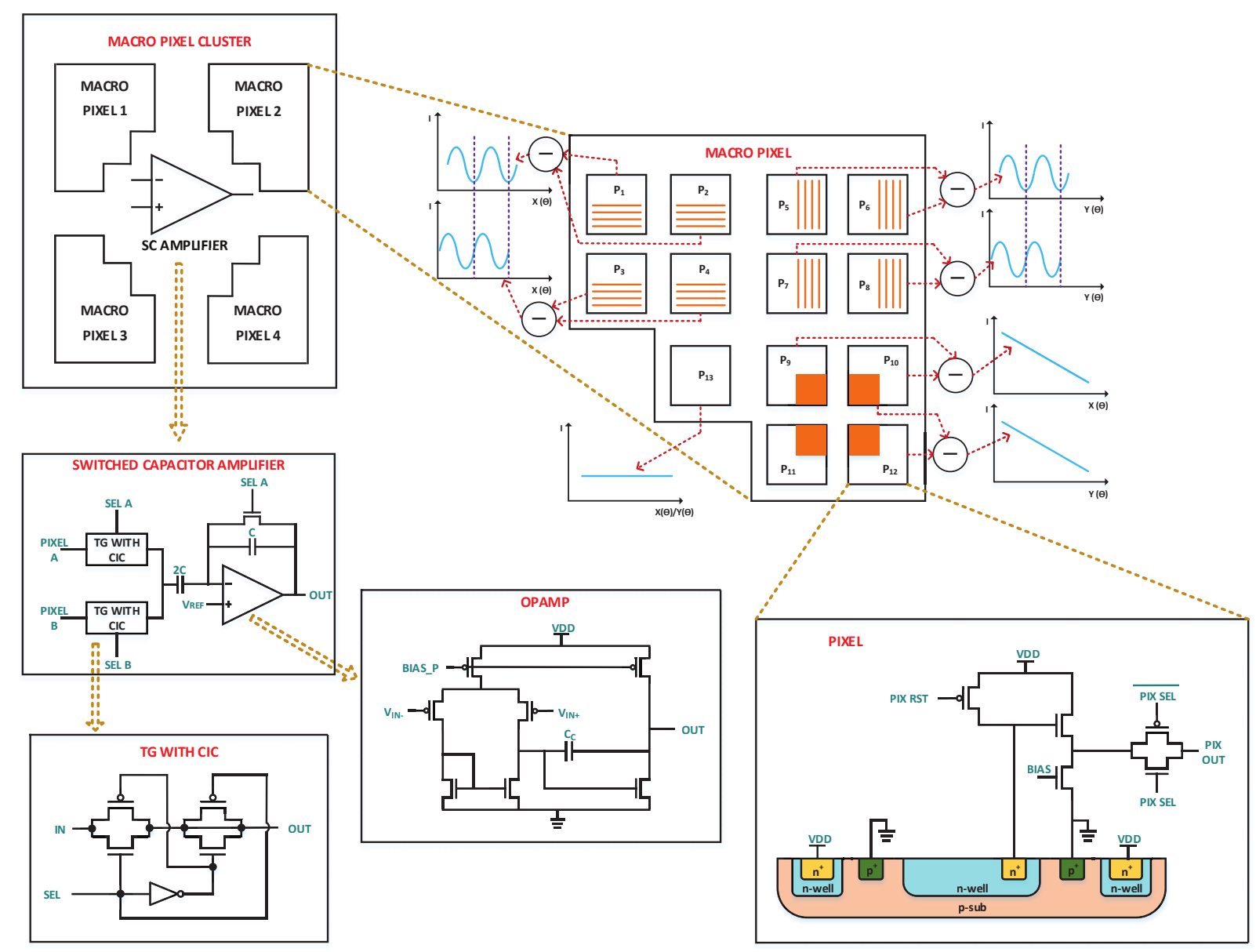

Figure 4.11: Sensor schematic showing the various structural components of the fabricated sensor. The various pixel types and their ideal responses are also shown (refer Table 4.1 for different pixel types).

$$
\mathrm{V}_{\mathrm{OUT}}=\mathrm{V}_{\mathrm{REF}}-\frac{2 \mathrm{C}}{\mathrm{C}}\left(\mathrm{V}_{\text {PIXELB }}-\mathrm{V}_{\text {PIXELA }}\right)
$$

N-well/p-sub photodiode makes up the light sensing part of the pixel. Each pixel measures $12.5 \mu \mathrm{m} \times 9 \mu \mathrm{m}$ and consists of 5 transistors. Three transistors along with the photodiode make up the conventional 3T APS structure and the other two transistors are for selection control. Each pixel additionally contain a n-well guard ring and a p-sub guard ring for better isolation. The effective photo sensing area is $6 \mu \mathrm{m} \times 6 \mu \mathrm{m}$, giving rise to a fill factor of $32 \%$. 
All the primary and secondary Talbot pixel gratings have a pitch of $0.76 \mathrm{um}$. This pitch was selected in order to produce optimum response for a design wavelength of 532 $\mathrm{nm}$ in vacuum. M5 metal layer acts as the primary grating and M1 metal layer acts as the secondary grating. For QPC, metal block is made of metal layer M5 and it covers $50 \%$ of the photodiode.

Important sensor characteristics are listed in Table 4.2. Photodiode sensitivity is the strength of signal developed by the pixel per unit of input optical energy and is measured for intensity pixels. Angular sensitivity on the other hand, is a measure of the angle dependent pixel response and is measured for Talbot and QPC pixels. Angular periodicity is the unique range of angles that can be measured and is a characteristic of the Talbot pixel as it produces a periodic response.

Table 4.1: Pixel Types in a Macro Pixel

\begin{tabular}{|c|c|c|c|}
\hline Pixel & $\begin{array}{c}\text { Metal } \\
\text { configuration }\end{array}$ & $\begin{array}{l}\text { Grat- } \\
\text { ing } \\
\text { offset }\end{array}$ & Response type \\
\hline $\mathrm{P}_{1}$ & \multirow{4}{*}{ Horizontal grating } & 0 & Cosine - offset 0 \\
\hline $\mathrm{P}_{2}$ & & $\mathrm{~d} / 2$ & Cosine - offset $\pi$ \\
\hline $\mathrm{P}_{3}$ & & $\mathrm{~d} / 4$ & Cosine - offset $\pi / 2$ \\
\hline $\mathrm{P}_{4}$ & & $3 \mathrm{~d} / 4$ & $\begin{array}{c}\text { Cosine - offset } \\
3 \pi / 2\end{array}$ \\
\hline $\mathrm{P}_{5}$ & \multirow{4}{*}{ Vertical grating } & 0 & Cosine - offset 0 \\
\hline $\mathrm{P}_{6}$ & & $\mathrm{~d} / 2$ & Cosine - offset $\pi$ \\
\hline $\mathrm{P}_{7}$ & & $\mathrm{~d} / 4$ & Cosine - offset $\pi / 2$ \\
\hline $\mathrm{P}_{8}$ & & $3 \mathrm{~d} / 4$ & $\begin{array}{c}\text { Cosine - offset } \\
3 \pi / 2\end{array}$ \\
\hline $\mathrm{P}_{9}$ & \multirow{4}{*}{ Metal block } & - & Linear \\
\hline $\mathrm{P}_{10}$ & & - & Linear \\
\hline $\mathrm{P}_{11}$ & & - & Linear \\
\hline $\mathrm{P}_{12}$ & & - & Linear \\
\hline $\mathrm{P}_{13}$ & No metal & - & Intensity \\
\hline
\end{tabular}




\subsubsection{Results and Discussion}

Fig. 4.12 shows the conceptual diagram of the test setup which consists of a sun simulator and a rotary board. The sun simulator emits highly collimated light rays toward the sensor. The sensor is mounted on a rotary board which allows for its rotation with an accuracy of $5^{\circ}$. The sun simulator emits light over a broad range of wavelengths covering the entire visible spectrum (300 nm - $700 \mathrm{~nm})$.

Table 4.2: Important Sensor Parameters

\begin{tabular}{|c|c|}
\hline Parameter & Value \\
\hline Technology & $\begin{array}{c}65 \mathrm{~nm}, \mathrm{CMOS} \\
\text { mixed-signal } \\
\text { process }\end{array}$ \\
\hline Chip size & $1.6 \mathrm{~mm} \times 1 \mathrm{~mm}$ \\
\hline Pixel size & $12.5 \mu \mathrm{m} \times 9 \mu \mathrm{m}$ \\
\hline Fill factor & $32 \%$ \\
\hline Pixel structure & $3 \mathrm{~T}$ APS \\
\hline Pixel power consumption & $10.13 \mu \mathrm{W}$ \\
\hline Dark current & $58 \mathrm{mV} / \mathrm{sec}$ \\
\hline Temporal noise & $0.54 \mathrm{mV}$ \\
\hline Dynamic range & $71 \mathrm{~dB}$ \\
\hline Full well capacity & $68,000 \mathrm{e}^{-}$ \\
\hline Conversion gain & $28.5 \mu \mathrm{V} / \mathrm{e}^{-}$ \\
\hline Photodiode Sensitivity & $0.53 \mathrm{~V} /$ lux-sec \\
\hline $\begin{array}{c}\text { Angular Sensitivity at } \\
\lambda=500 \mathrm{~nm} \text { (Talbot pixel) }\end{array}$ & $14.07 \mathrm{mV} / \mathrm{deg}$ \\
\hline $\begin{array}{c}\text { Angular Sensitivity at } \\
\lambda=500 \mathrm{~nm}(\mathrm{QPC} \text { pixel })\end{array}$ & $2.918 \mathrm{mV} / \mathrm{deg}$ \\
\hline $\begin{array}{l}\text { Angular Periodicity (Talbot } \\
\text { pixel) }\end{array}$ & $20 \mathrm{deg}$ \\
\hline Array size & $\begin{array}{c}(12 \times 10) \times(4 \times \\
13)\end{array}$ \\
\hline Supply voltage & $\begin{array}{l}3.3 \mathrm{~V} \text { analog; } \\
1.2 \mathrm{~V} \text { digital }\end{array}$ \\
\hline
\end{tabular}

The image sensor is interfaced with an external serial ADC and requires regulated $3.3 \mathrm{~V}$ and $1.2 \mathrm{~V}$ to function properly. The sensor communicates with the PC through 


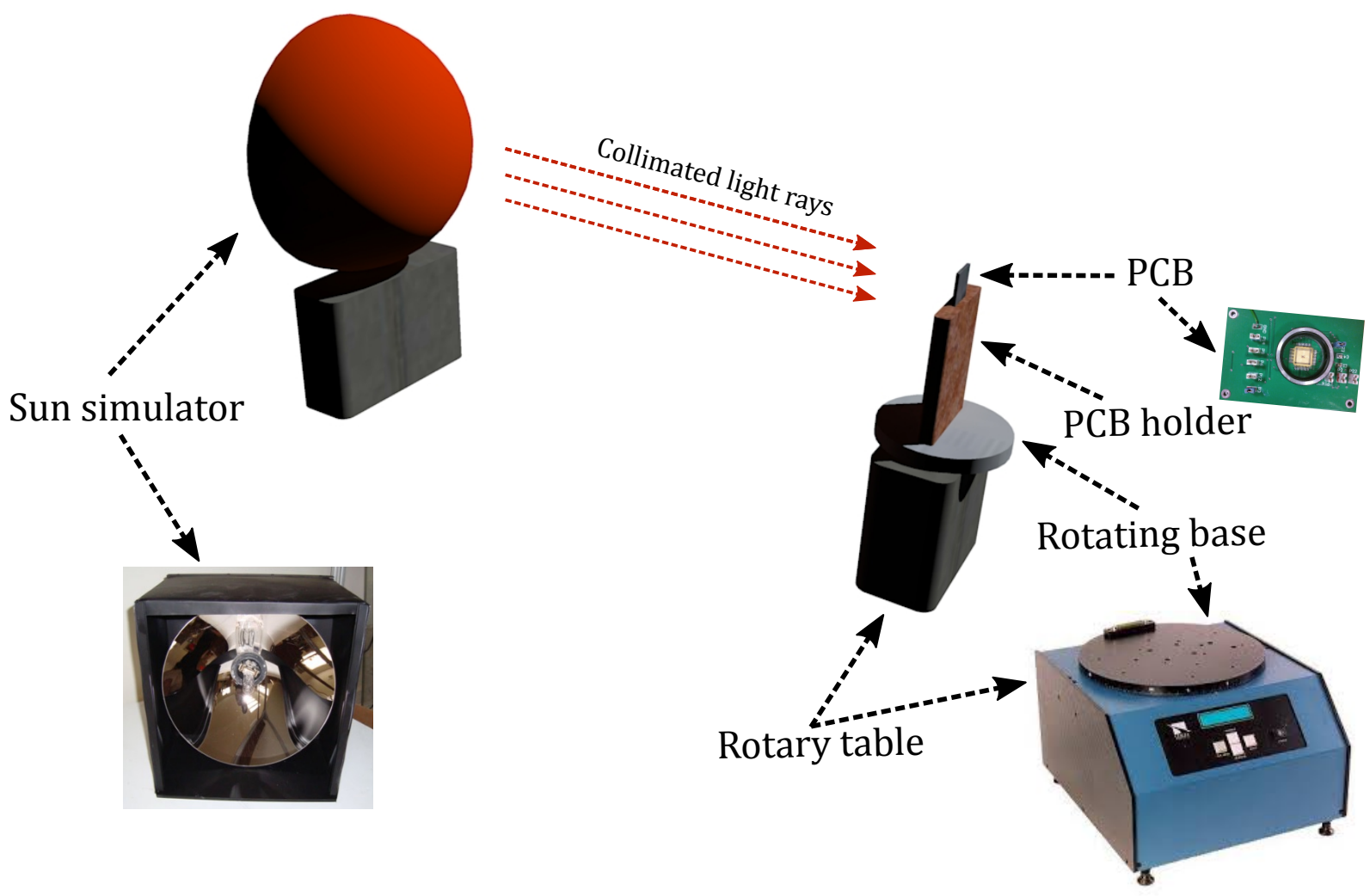

Figure 4.12: Conceptual diagram of the test setup.

an Opal Kelly XEM 3010 integration board which consists of an FPGA for generating control signals, a SDRAM for storing output digital values from the ADC and many other peripheral circuits.

Measurements were made, first without any optical filter over the sensor and then with a $500 \mathrm{~nm}$ filter that has a passband of $\pm 2 \mathrm{~nm}$ around $500 \mathrm{~nm}$. For both cases, angles were first varied along the horizontal (X) direction and then along the vertical (Y) direction. Average values of pixels of similar kind over the entire pixel array are plotted (Fig. 4.13). Fig. 4.13(a) and Fig. 4.13(b) show the pixel responses without any optical filter and Fig. 4.13(c) and Fig. 4.13(d) show the pixel responses with a $500 \mathrm{~nm}$ optical filter on top of the sensor. In all the cases, it is the difference response that has been plotted. The various pixel types shown in the plots are given in Fig. 4.11 and Table 4.1. The experimental QPC values were subjected to a polynomial fit of degree 2 and it is 
this data that has been plotted.

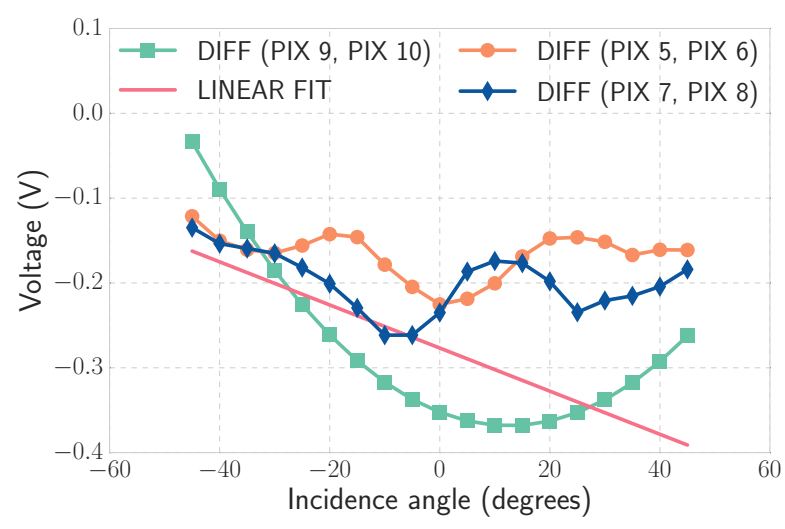

4.13.a: Pixel response without filter (angle variation along $\mathrm{X}$ direction).

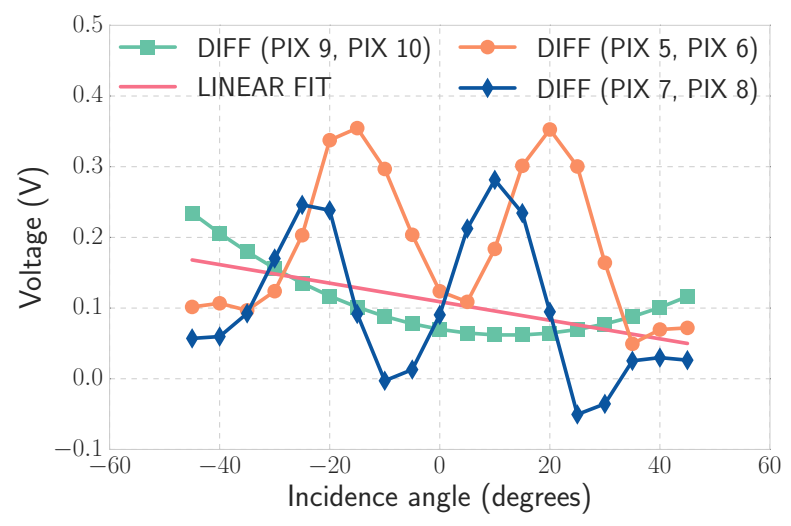

4.13.c: Pixel response with $500 \mathrm{~nm}$ filter (angle variation along $\mathrm{X}$ direction).

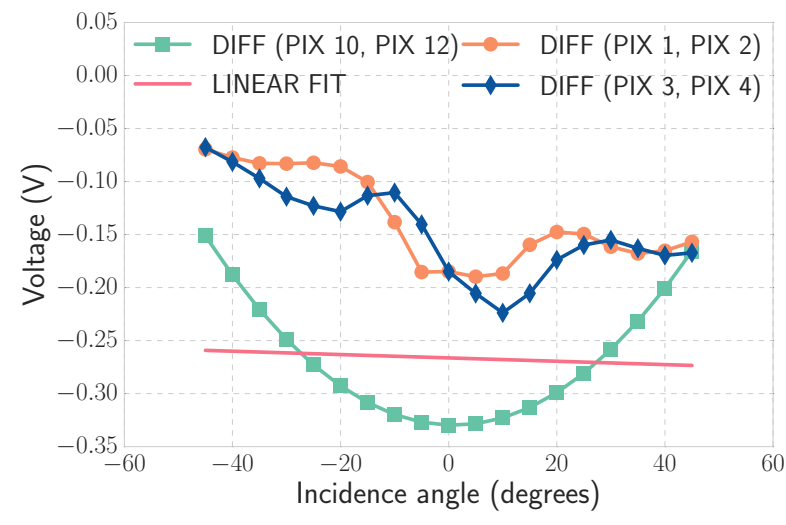

4.13.b: Pixel response without filter (angle variation along $\mathrm{Y}$ direction).

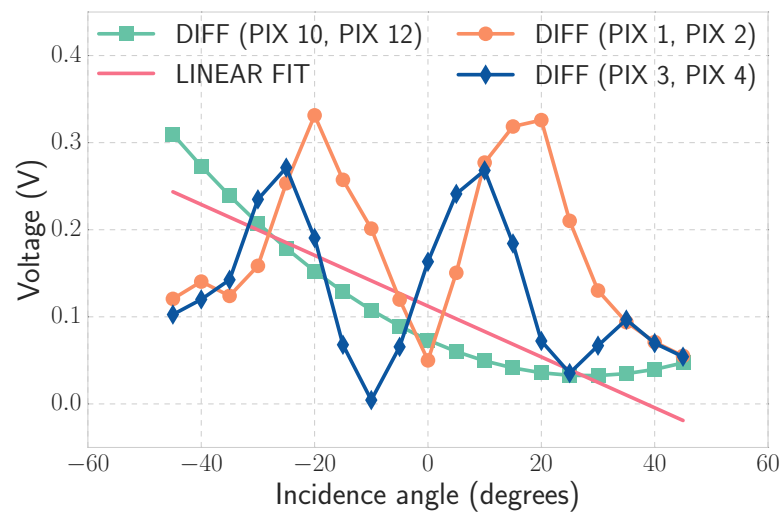

4.13.d: Pixel response with $500 \mathrm{~nm}$ filter (angle variation along $\mathrm{Y}$ direction).

Figure 4.13: Plot of measured pixel responses as the incidence angle is varied from $-45^{\circ}$ to $+45^{\circ}$. Different pixel types and ideal responses are shown in Fig. 4.11 and Table 4.1

Possible reasons for parabolic nature of the QPC response curves are light refraction from multiple dielectric layers in the CMOS metal stack and shadowing of pixels from metal layers in the periphery of the pixels. The anomaly can be corrected to a certain extent to obtain linear response by using the intensity pixel response (scale the intensity pixel response and subtract it from QPC pixel response). The "LINEAR FIT" line shows the general linear trend of the QPC response. 
In order to determine the angle of incident light, we first look at the QPC response to get a coarse estimate. We then corroborate the result by looking at the Talbot pixel response, which also refines the angle to a finer value. As an example consider Fig. 4.13(a), which shows the QPC and Talbot pixel response for angle variation along the X (horizontal) direction. We can use any one of the curves (either DIFF (PIX5, PIX6) or DIFF (PIX7, PIX8)) for fine angle measurement, as both contain the same information, albeit with a small offset. Let us consider DIFF (PIX5, PIX6) for angle detection. Fig. 4.14 shows the experimental determination of angle. Only relevant waveforms from Fig. 4.13(a) have been reproduced in Fig. 4.14.

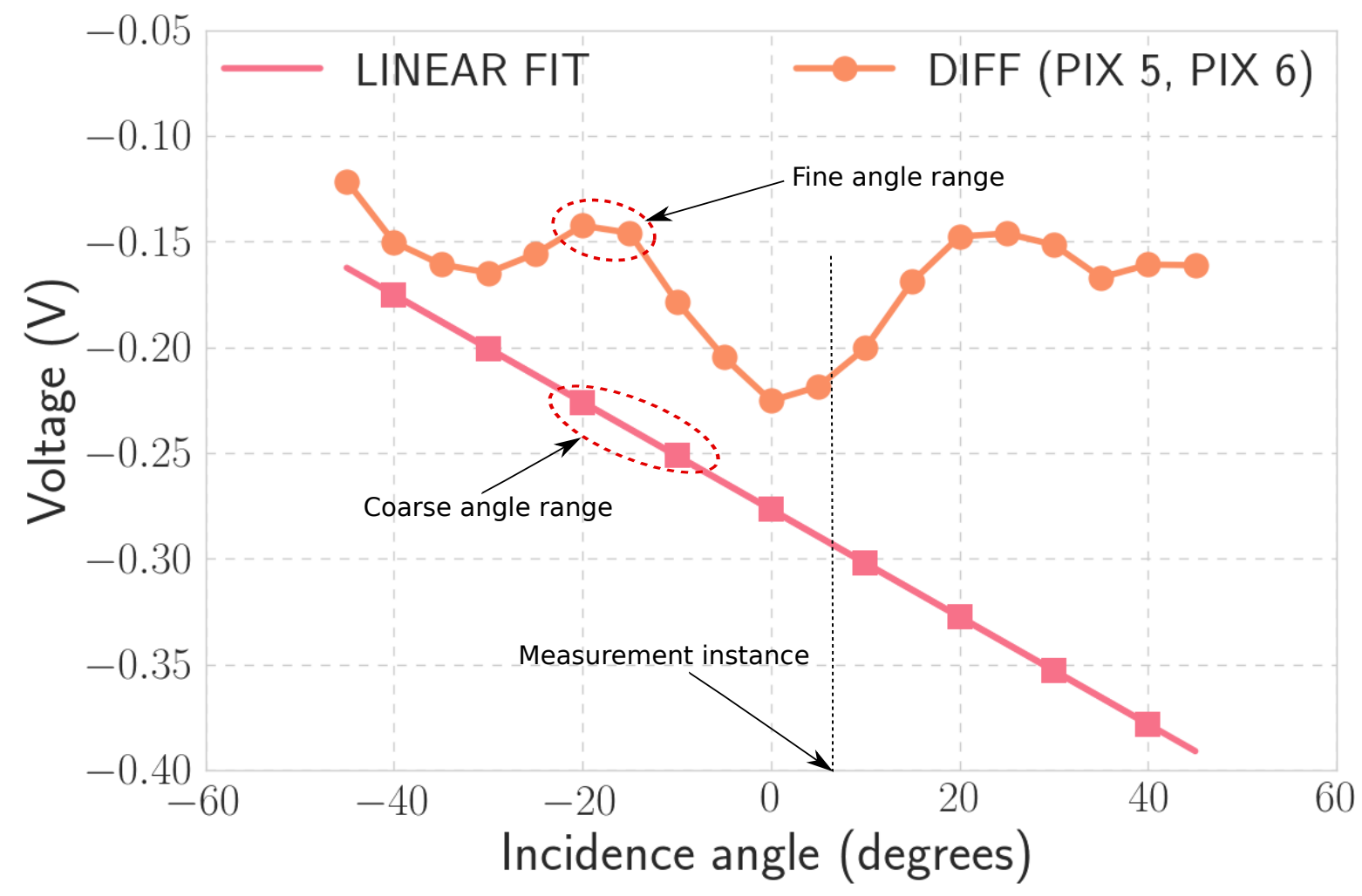

Figure 4.14: Figure showing measurement of angle from experimental data.

In the figure "Measurement instance" represents the time instant at which the angle information is captured. At this instant, QPC pixels have voltage corresponding to the 
coarse angle value and Talbot pixels have voltage corresponding to the fine angle value. Since the angular sensitivity of QPC pixels are low, their angular resolution is low. For the present case, let us suppose that they can distinguish between angles over a $10^{\circ}$ increment between $-45^{\circ}$ to $+45^{\circ}$. This is indicated by the "Coarse angle range" in the figure. In order to determine the exact angle value within a $10^{\circ}$ angular range we need fine resolution. This is provided by Talbot pixels that can distinguish fine angles, say over a $5^{\circ}$ increment $\left(5^{\circ}\right.$ is just an example, the actual value is limited by the accuracy of the measurement setup and readout circuits). This is indicated by "Fine angle range" in the figure.

Suppose we have light at an angle around $10^{\circ}$, the QPC pixel indicates that the angle is $10^{\circ}$ (considering that the angle resolution capability of QPC pixel is $10^{\circ}$ ). This could either be the actual angle or the actual angle might be somewhere near this angle. We then proceed to see the response produced by Talbot pixel. Since Talbot pixels have greater angular sensitivity, they can resolve angles with a much finer resolution, $5^{\circ}$ or even $1^{\circ}$. Fig. 4.14 shows Talbot pixels with an angular resolution of $5^{\circ}$. In the absence of a reference angle, such as that provided by QPC pixel, there would be ambiguities in the measurement of angles using only the Talbot pixel response. For example, in Fig. 4.14 , both $-5^{\circ}$ and $+5^{\circ}$ have approximately the same voltage values. This would require additional Talbot pixels with different angular sensitivities to resolve the ambiguity [88].

In the present case, we use guidance provided by the QPC response to aid in determination of incident angles. Since the angle from QPC response is $10^{\circ}$, we can consider the Talbot response around this angular range to determine the exact angle. By examining the Talbot response we see that the actual angle is in fact $+5^{\circ}$ (considering that the angle resolution capability of Talbot pixel is $5^{\circ}$ ).

The imaging scene generally contains light at different wavelengths. The wavelength dependent response produced by the Talbot only design makes it unsuitable for practical 
applications. The reduced response strength (Fig. 4.13(a) and 4.13(b)) without filters prove this point. In the presence of a broadband source emitting light over a broad range of wavelengths, the grating produces self-images at a particular depth, dependent on the characteristic wavelength. The response perceived by the photodiode for such a source will be the resultant of all the characteristic responses. Hence a solution consisting of coarse angle tracking by QPC and fine angle tuning by Talbot pixel is the easiest way out. The technique can be employed satisfactorily for a wide variety of applications.

\subsection{Enhanced Quadrature Pixel Cluster}

Figure 4.15 shows an alternative arrangement of metal mask to produce a quadrature pixel cluster with high angular sensitivity. Although this structure blocks more of the light incident on the pixel, it has a higher angle sensitivity owing to the larger edge of the metal over the photodiode. The principle of angle detection remains the same in this case as that of the ordinary quadrature pixel cluster.

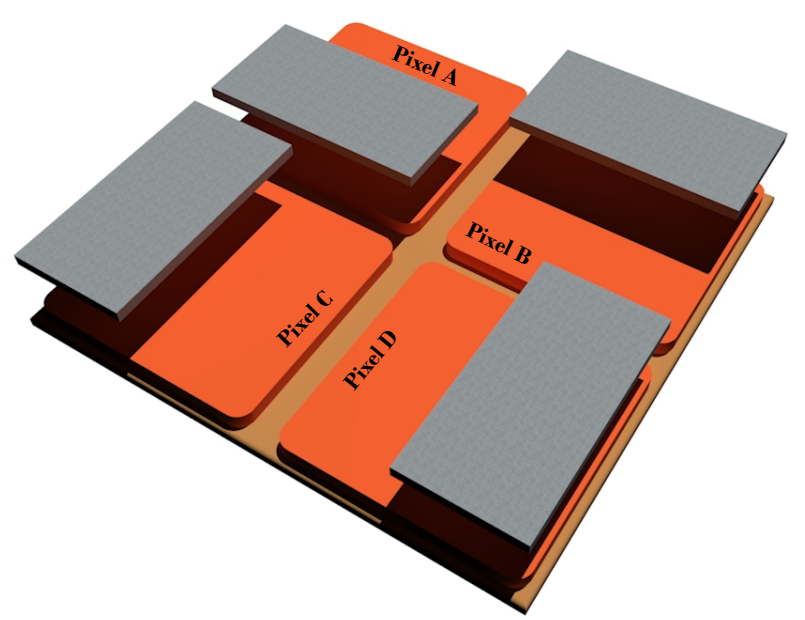

Figure 4.15: Physical structure of enhanced angle sensitive quadrature pixel cluster.

Figure 4.16 shows the results from the FDTD simulations for enhanced QPC pixels. Here QPC bottomMET refers to Pixel A which has the metal mask at the bottom 
and QPC topMET refers to Pixel B which has the metal mask at the top. The QPC difference is the difference response between Pixel A and Pixel B.The difference is linear from $-20^{\circ}$ to $+20^{\circ}$. Figure 4.17 shows the comparison between the QPC and enhanced QPC pixel responses. The enhanced QPC has more angle sensitivity especially for large angle deviations from the normal $\left(0^{\circ}\right)$.

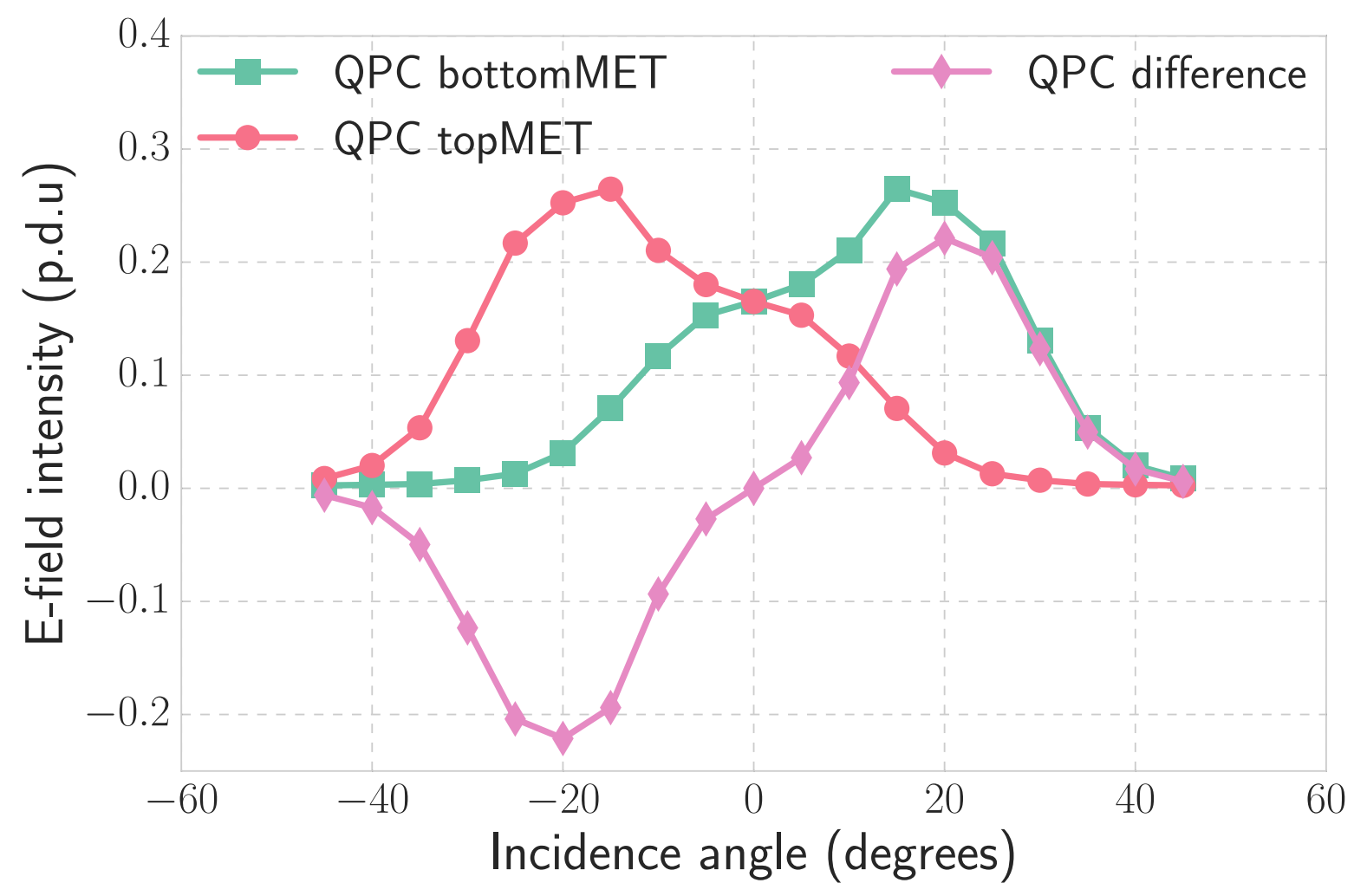

Figure 4.16: Response of enhanced QPC pixels and their difference.

From our experiments with a fabricated prototype sensor in $0.35 \mu \mathrm{m}$ CMOS process we observed that the sensitivity for ambient light conditions is not very high for angle detection targeting general imaging applications. One way to increase the sensitivity is to use a micro-lens on top each of the QPC pixels as shown in figures 4.18.

QPC and enhanced QPC pixels use four pixels for incident light angle detection. In an effort to push angle sensitive imaging to the extreme we propose an alternative angle 


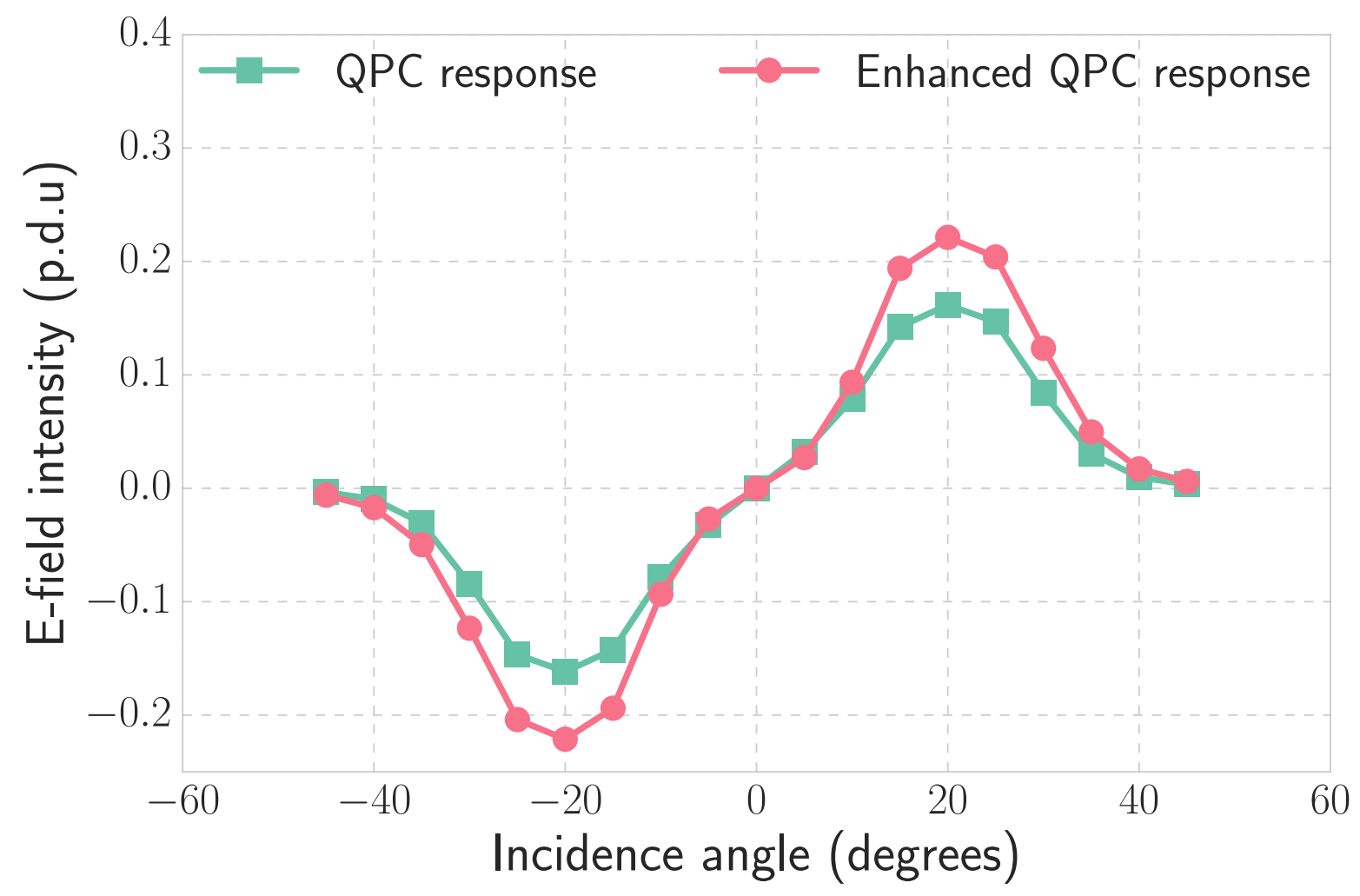

Figure 4.17: Comparing angle sensitivity of QPC and enhanced QPC pixels.

\section{Light Source}

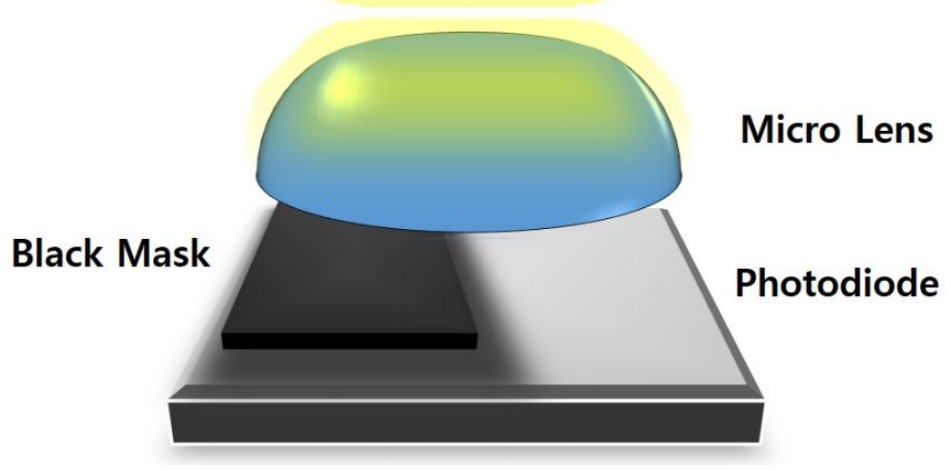

Figure 4.18: Enhanced QPC pixel with microlens [39]

sensitive pixel structure, which we call multi-fingered pixel, as it is fabricated in islands of N-well/P-sub with isolated metal gratings on top of them. We explore three such pixel 


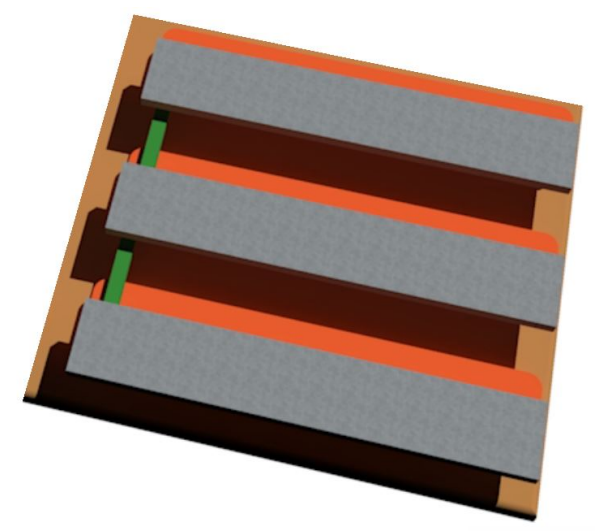

Figure 4.19: Single multi-finger pixel.

structures, and one of these form the basis for our imaging tests of chapter 6 .

\subsection{Multi-Finger Pixel Design}

Unlike Talbot pixels, which depend on micron scale diffraction effects to produce angle sensitive response for incident light, Multi-Finger pixels make use of asymmetry in the pixel structure. The angle sensitive response in these pixels is a macro scale manifestation of the shading induced by the metal mask and the arrangement of the N-well/P-sub photodiode islands. The arrangement is similar to the one used in the enhanced Talbot pixel [72], but at a macro level. The use of $\mathrm{P}+/ \mathrm{N}$-well juction in [72] result in a loss of quantum efficiency (QE) of the pixel due to the shallow depth of the photodiode junctions. Photodiodes produced using these junctions are also know to be more sensitive to the shorter light wavelengths unlike the ones with the N-well/P-sub junctions which are more sensitive to the mid band wavelengths (green light).

Figure 4.19 shows a horizontal multi-finger pixel with three N-well/P-sub islands with light obstructing metal masks on top of each. The three photodiode (PD) islands are connected together electrically and the pixel response that is read out of the pixel is the combined one.

Figure 4.20 shows the FDTD simulation results for the MF pixel. It shows the 


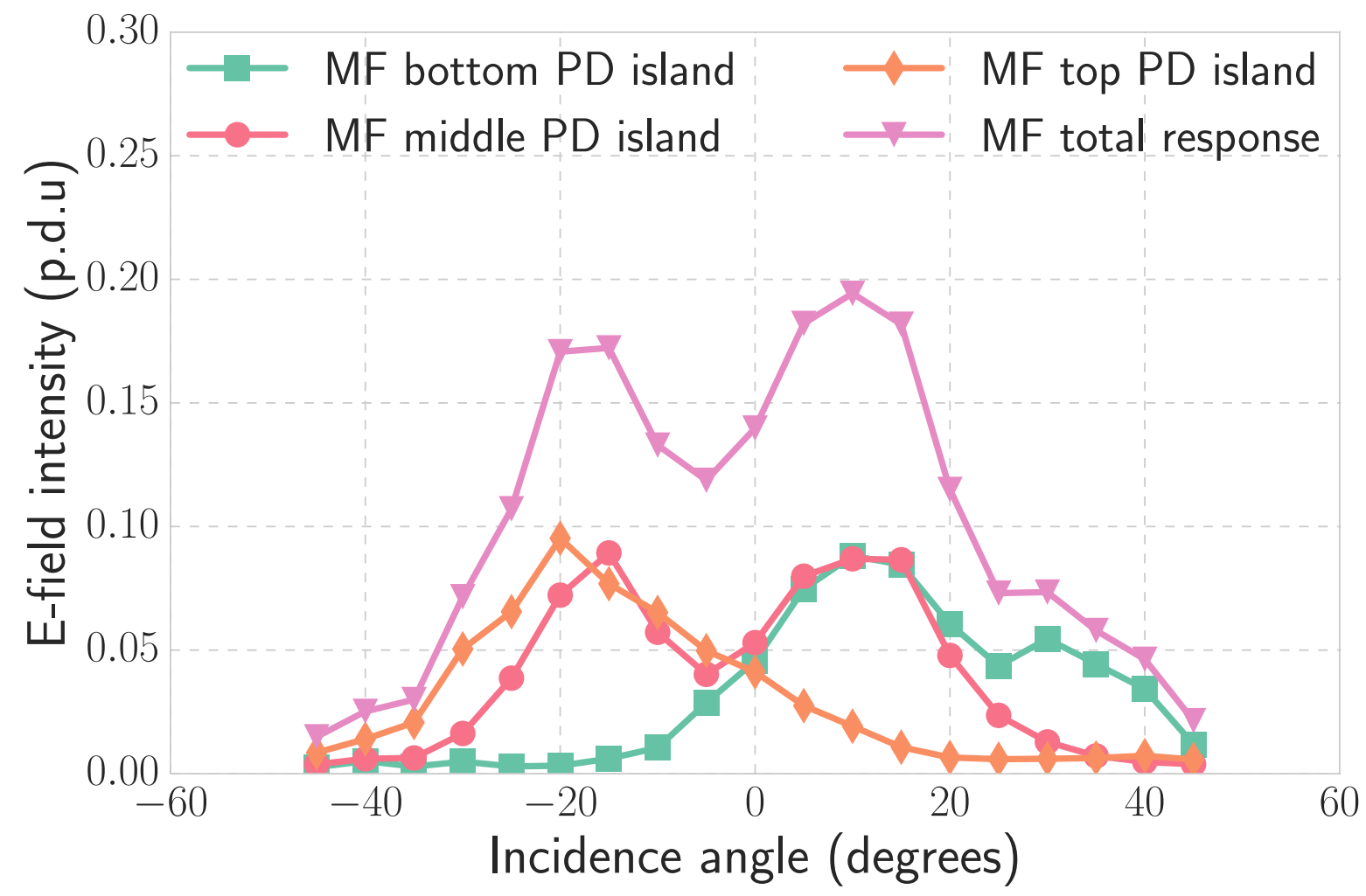

Figure 4.20: Response produced by a horizontal multi-finger pixel for vertical light angle variation.

response produced by the individual PD islands and the combined response that is read out from the fabricated pixel array. The total pixel response is the characteristic pixel response modulated by the pixel envelope function which is a consequence of the Lamberts cosine law.

This pixel modulates the light rays arriving at its focal plane to induce an angle sensitive response. When we take a closer look at the response produced by the individual PD islands we see that the response produced by the bottom and the top islands are of the same nature as that of the metal shading pixels, albeit with a loss of quantum efficiency due to the gaps between the islands and their small footprint. The middle PD island however produces a sinusoidal response mimicking the response of the top PD island during negative light incidence and mimicking the response of the bottom PD island 
during positive light incidence.

The final response which is the result of integrating the response from all the PD islands is sinusoidal in nature with the total intensity and angle sensitivity greater than the individual PD islands.

Since the response of the MF pixel is similar to that of the Talbot pixel, we can approximate it with the same parameters as that of the Talbot pixels. Based on this empirical evidence the formula can be approximated by equation Eq. 4.14.

$$
I=I_{0}\left(1+m_{M F} \cos \left(\beta_{M F} \theta+\alpha\right)\right)
$$

The parameters $m_{M F}$ and $\beta_{M F}$ hold the same meaning as that of that of the Talbot response. $m_{M F}$ represents the strength of the response and depends on the metal thickness and metal overlap over the photodiode area. $\beta_{M F}$ represents the periodicity of the response and depends on the height of the metal mask above the photodiode. $\alpha$ represents the shift of the metal mask with respect to the photodiode, a complete overlap is denoted by $\alpha=\pi / 2$ and no overlap is denoted by $\alpha=0$.

The response depends on a number of factors, such as number of islands, metal layer (thickness and height) used on top of the islands, position of the metal masks with respect to the $\mathrm{PD}$ islands, width and spacing of the $\mathrm{PD}$ islands. The dependence of the response on these parameters can be easily understood by comparing it with the analysis of the QPC pixel presented in the earlier sections. Other minor factors such as manufacturing tolerances, refraction at the surfaces of the multiple passivation layers, presence of multiple dielectric layers, etc., produce variations in the results. But since this is a macro structure, as opposed to the Talbot micro structures, the tolerance for inconsistencies are greater. 


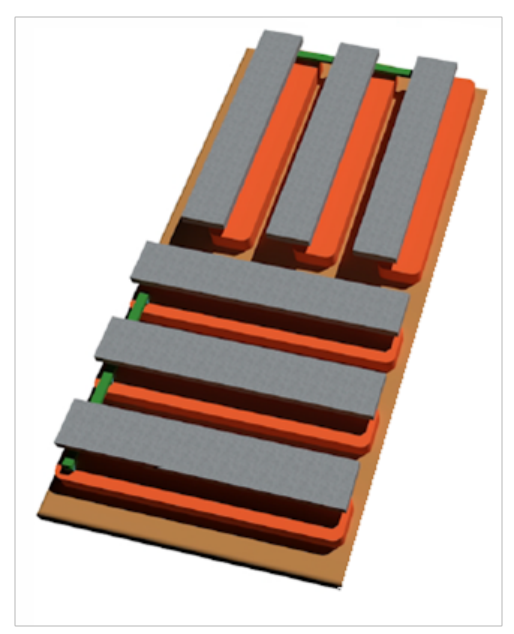

Figure 4.21: Physical structure of orthogonal multi-finger pixels.

\subsection{Orthogonal Multi-Fingered Pixels}

The response produced by a multi-finger pixel is only sensitive to angle variations orthogonal to the direction of the fingers. In order to provide a greater angle variance we have implemented multi-finger pixels with two orthogonal orientations - horizontal and vertical (figure 4.21). As we show in the chapter 6 we can generalize local angle detection by combining it with edge detection.

In order to put things in perspective and bring together the ideas that were presented in the thesis, we designed an angle sensitive image sensor in $0.35 \mu \mathrm{m}$ AMS process. The sensor is based on the orthogonal multi-fingered pixels and measures $7.5 \mathrm{~mm} \mathrm{x} 7 \mathrm{~mm}$. It contains a total of 96,256 pixels arranged in 256 rows and 376 columns. Each of the pixels are either a horizontal or vertical multi-fingered pixel, with the pair acting as a group for local incident angle detection.

Figure 4.22.a shows the chip microphotograph of the multi-finger sensor. Each pixel measures $15 \mu \mathrm{m} \times 15 \mu \mathrm{m}$ and is made up of a N-well/P-sub photodiode and 3 transistors forming the active pixel sensor (APS). Figure 4.22.b shows the chip architecture with with its various blocks labeled. The sensor undergoes row-wise exposure and the integrated charge is converted to voltage by the in-pixel source follower and is passed onto the 
correlated double sampling (CDS) circuits for canceling the column fixed pattern noise $(\mathrm{FPN})$. In fact the circuits perform pseudo FPN with the pixel voltage being read out to the CDS circuits before the pseudo reset voltage. This is unlike true FPN that the 4T APS pixels facilitate due to their floating diffusion. Although the reset and pixel voltage is not fully correlated in pseudo FPN, noise is partially correlated and it is better to have the CDS circuits than not having it.

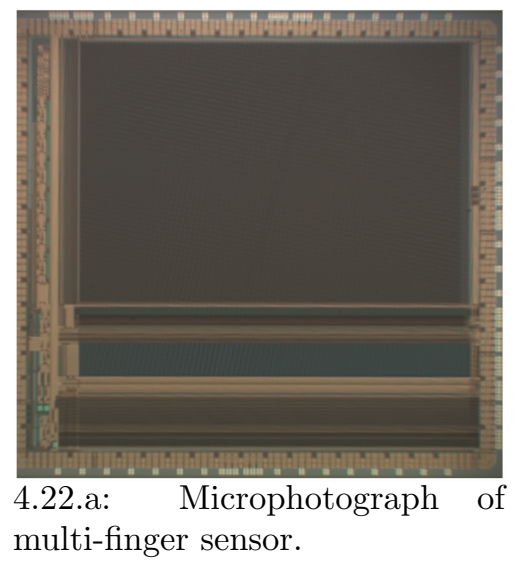

multi-finger sensor.

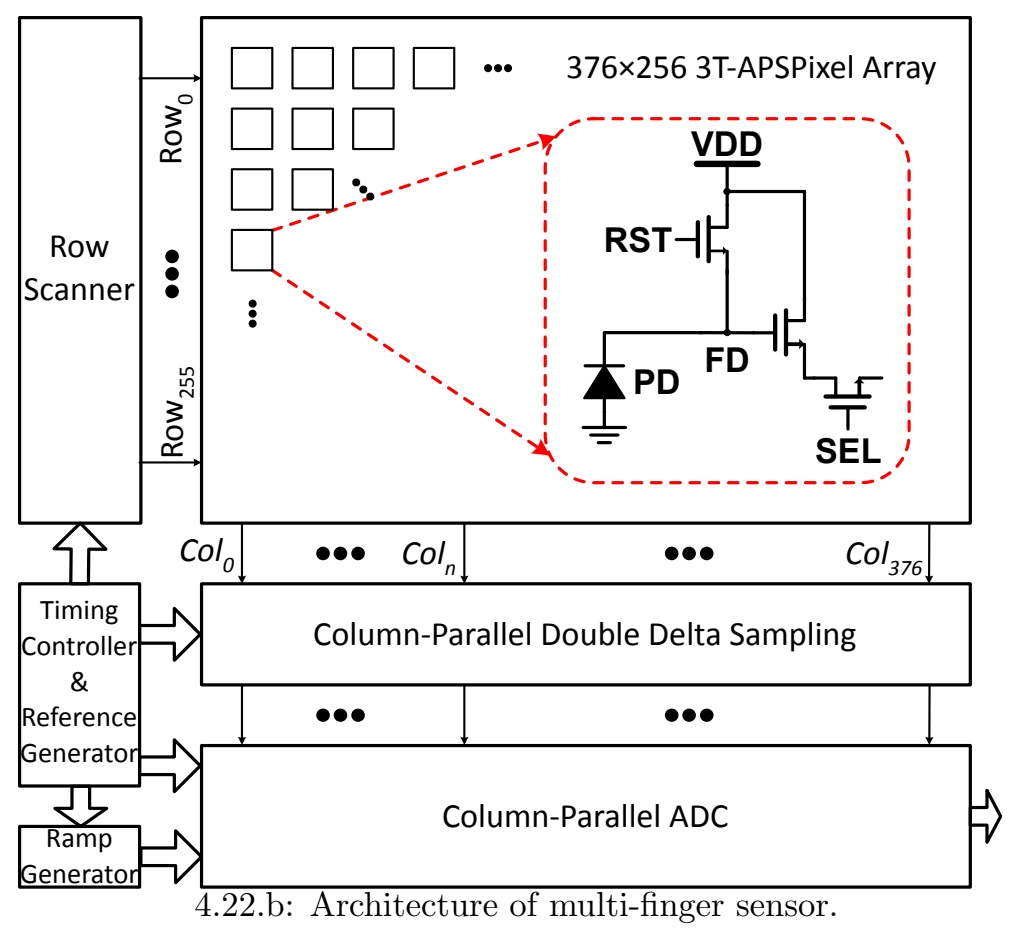

Figure 4.22: Multi-finger angle sensitive image sensor.

The FPN canceled pixel voltage is passed onto a 11-bit SAR (Successive Approximation Register) column-parallel ADC (Analog to Digital Converter), which digitizes the voltage facilitating digital readout. We then use a custom designed frame grabber to send this digitized data to a computer for further downstream processing. The control signals for the sensor are provided by an FPGA present in the frame grabber.

Figure 4.23 shows the results for the angle dependent tests that we conducted on the designed sensor. The test setup we used is similar to the one used earlier. The 


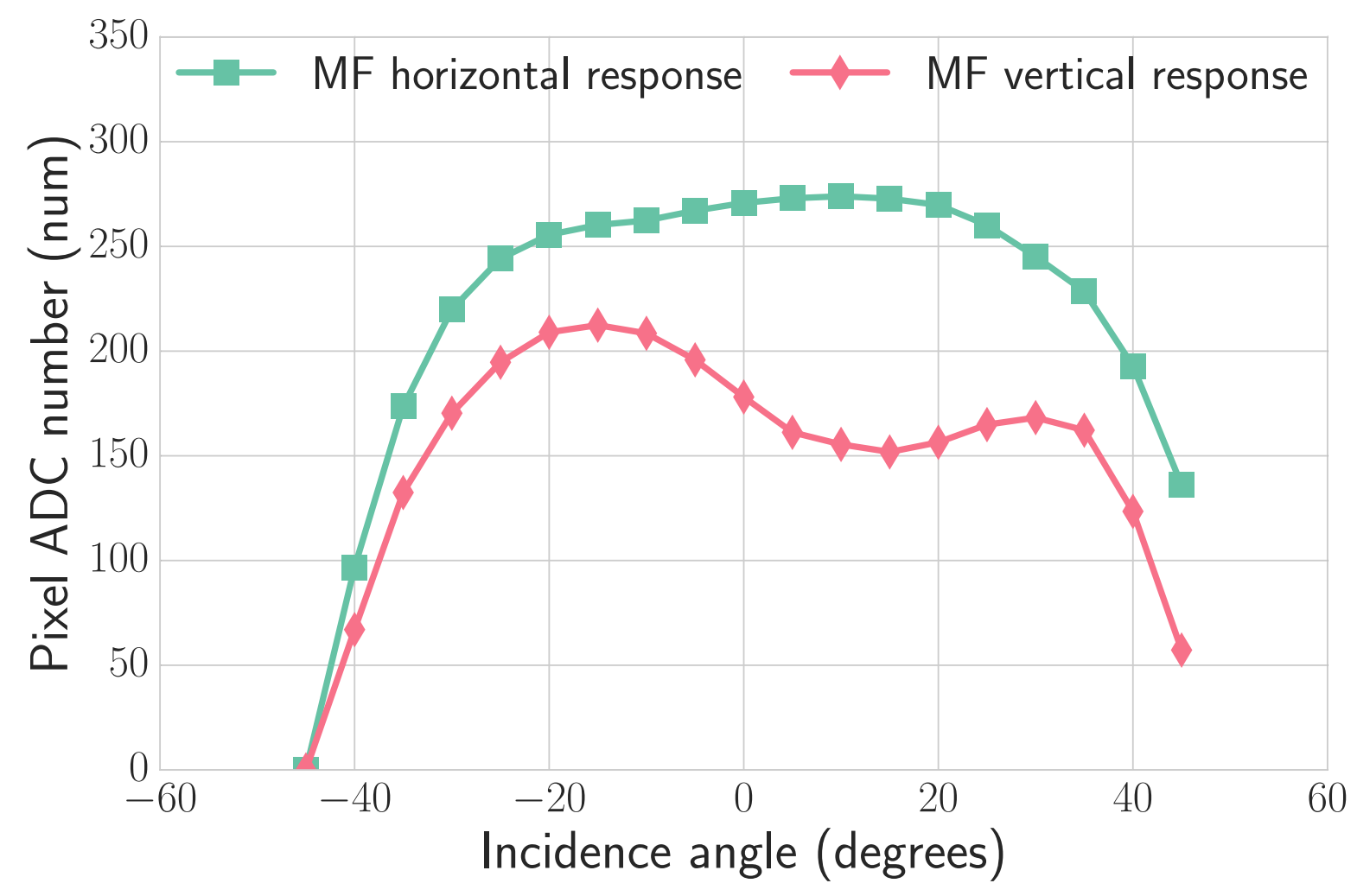

Figure 4.23: Response of orthogonal multi-finger pixels.

sensor was placed on a rotary board and was facing a broad-band collimated light source (sun simulator). The angle between the sun simulator and the sensor was varied by rotating the rotary board in steps of 5 degrees. The result is plotted in figure 4.23. The horizontal pixel response is relatively insensitive to angle variations whereas the vertical pixel response varies as a sine function. The responses are modulated by pixel envelope function (a result of the presence of metal structures in the periphery of the photodiode and a consequence of Lambert's consine law for oblique light angle incidence), which explains the intensity falloff at steeper angle variations. 


\subsection{Antisymmetric Multi-Fingered Pixels}

One could use an alternate pixel arrangement as shown in figure 4.24(a) to increase the angle sensitivity of the angular response produced by the image sensor.

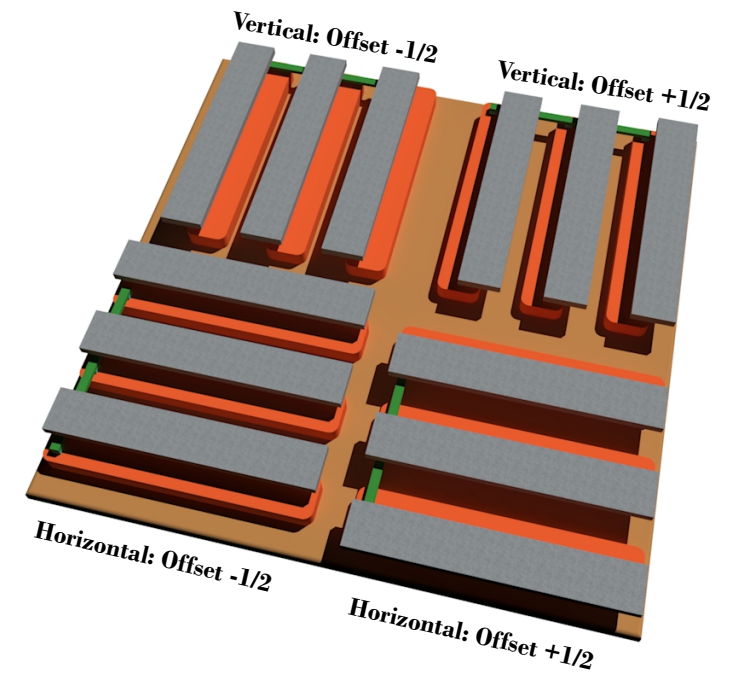

4.24.a: Pixel structure

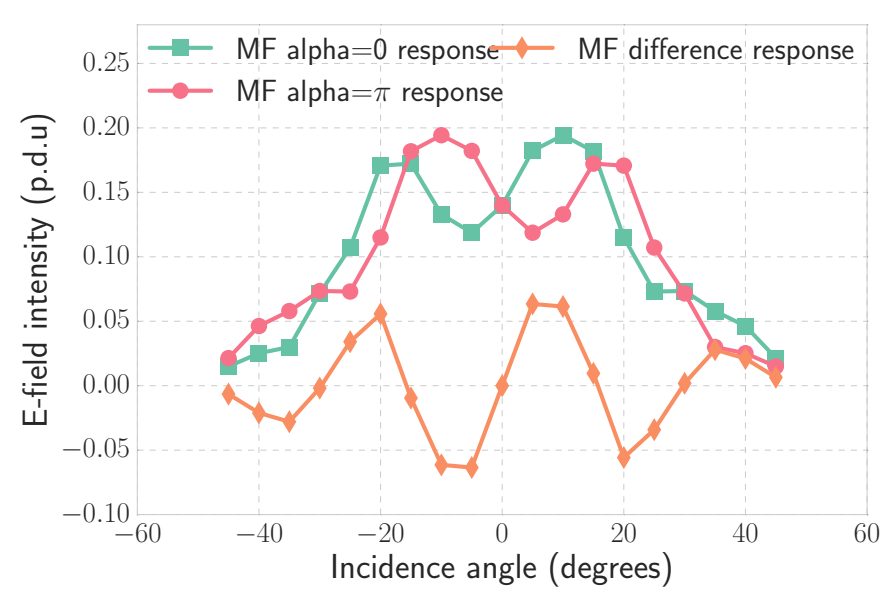

4.24.b: Pixel response

Figure 4.24: Antisymmetric MF pixel structure and response.

This technique uses two antisymmetric pixels to produce complementary responses (figure 4.24(a)), whose difference produces the final output. Although the angular sensitivity is doubled, the spatial resolution is reduced by half. Figure 4.24(b) shows the FDTD simulation results for the response produced by two antisymmetric pixels and their difference. 


\section{Chapter 5}

\section{Polarization-Incident Angle Sensitive Imaging}

In this chapter we explore a hybrid angle sensitive pixel design using polarization pixels. These pixels are capable of responding to polarization and incident angle changes in light.

Polarization is a property of Electro-Magnetic (EM) waves (which includes visible light) in which the electric field (or magnetic field) has preference for vibration along a particular orientation.

EM waves are said to be unpolarized or randomly polarized (Fig. 5.1a) if the electric field does not have any preference for vibration along a particular orientation. On the other hand, if the electric field is confined to vibrate along a particular orientation, the light is said to be linearly polarized. EM waves are said to be partially polarized (Fig. $5.1 b)$ if they have a predominant electric field component with a well defined vibration axis, along with other minor components along random orientations. Majority of manmade light sources are partially polarized [92]. Sun light gets partially polarized due to scattering on passing through earth's atmosphere. Light can also become polarized as a result of reflection from dielectric surfaces and refraction on passing through certain birefringent materials such as calcite.

Many animals have the ability to detect the polarization state of light in their natural habitats. Some arthropods like crayfish [29], [78], spider [50], desert ant [55], desert 


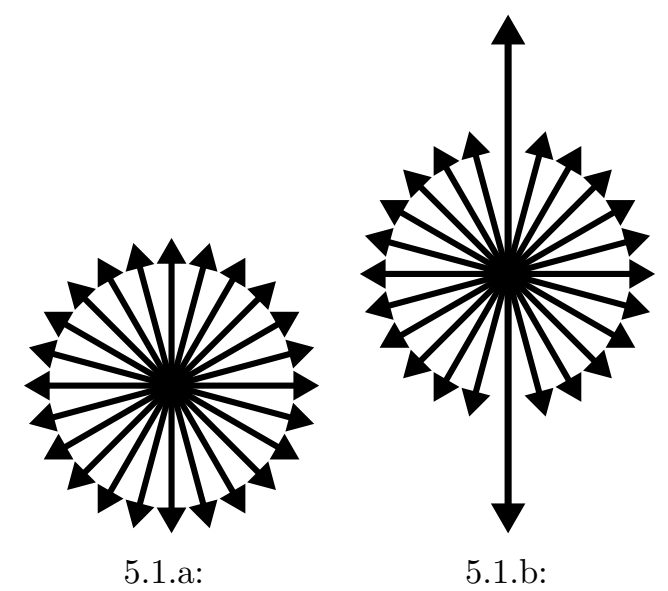

Figure 5.1: Figure showing electric-field vector orientation of randomly polarized light (a) and partially polarized light (b) with its major component oriented along the $90^{\circ}$ axis.

locust [35] and certain vertebrates like lizards [5] and salmon [32] are known to make use of polarization information in their surroundings for either egocentric navigation [94] or secondary vision that assists in polarization contrast imaging. Although humans have a well developed vision that detects variations in color and brightness over several orders of magnitude, they are incapable of detecting the polarization information.

Literature abounds with the many applications that are enabled by the capture of polarization information. Material classification [96], [66], navigation [68], polarization contrast imaging of biological tissues [49], non-contact latent fingerprint imaging [47], enhancing vision under hazy conditions [70], 3D object recognition [52] and many others. All these applications are enabled by the ability to detect the polarization information through CCD or CMOS cameras [7]. In order to make an ordinary imager detect polarization information, we need to augment them with special optics, known as polarizers.

Polarization state of the light can be detected by using an image sensor with pixels capable of producing response proportional to the polarization state. Division-of-time, division-of-focal-plane [43] and division-of-amplitude [23] are the prominent techniques for polarization detection using a CMOS/CCD image sensor. Division-of-time polariza- 
tion imaging is realized by using a rotating polarizer in front of the imager and capturing the image for each orientation of the polarizer in a time multiplexed manner. Multiple images must be captured and processed to determine the polarization state of the incident light. The main drawback of this technique is time aliasing for scenes with object motion or brightness variations.

Division-of-amplitude polarization imaging uses beam splitters and retarders along with two or more image sensors to capture the polarization information. Requirement of additional optical components and precise alignment of the optical setup is a serious limitation with this technique.

Division-of-focal-plane polarization imaging is realized by having polarization sensitive gratings on top of the pixel in the focal plane of the sensor. Neighboring pixels have gratings with different orientations and adjacent pixels work as a group to determine the local polarization information. The drawback here is a reduction in spatial resolution that is dependent on the number of grating orientations that are being realized in the pixel array.

Division-of-focal-plane polarization imaging is almost always preferred because of the above mentioned issues related to division-of-time and division-of-amplitude polarization imaging; even at the cost of reduced spatial resolution. This shift is also rendered possible by the miniaturization of pixel geometry as a result of technology scaling and the subsequent improvements in optical lithography enabling fabrication of polarizers with sufficiently small dimensions.

Traditionally, division-of-focal-plane polarizers consisted of polarization gratings made of poly vinyl alcohol (PVA) polymers, birefringent crystals or Aluminum nano-wires which were deposited on top of pixels from commercial CCD or CMOS image sensors through post-CMOS fabrication process.

Usually multiple sheets of polarizer films had to be placed to achieve multiple polarizer orientations [7]. This reduced the light transmission onto the photodiode, making signal 
detection challenging. These multiple sheets placed at a considerable height above the photodiode surface also increased pixel crosstalk leading to a reduction in the polarization extinction ratio (PER - is a measure of the quality of the polarizer) of the polarizer. PER is also sensitive to the alignment between the pixel and the polarizer sheets and care must be taken to avoid any misalignment. The cost for such a polarization imager increases as a result of all the post processing operations.

With the rapid scaling of CMOS technology over the past decade, implementing polarizer gratings monolithically on top of the pixel has become an option. These gratings are implemented in the metal layers of the CMOS process stack. This requires no additional post processing steps and careful design can reduce pixel crosstalk. However, unlike other techniques mentioned above, the on-chip polarizers generally tend to have a low extinction ratio. As the technology continues to scale and the minimum required metal width decreases, the extinction ratio will become large enough to be useful for a wide variety of polarization applications.

Figure 5.2 shows randomly polarized light being horizontally polarized as a result of it passing through a vertical wire-grid polarizer. When a randomly polarized light is incident on a wire-grid polarizer, all the components of the electric field along the orientation of the polarizer will be blocked by it while the components orthogonal to the polarizer orientation will be allowed to pass through.

A polarizer grating can be characterized by its width 'W' and pitch 'd'. From theory [33] we know that a wire grid grating such as the one shown in figure 5.2 is capable of exhibiting polarization properties if $\lambda>2 \mathrm{~d}$, where $\lambda$ is the wavelength of the incident light. In the $65 \mathrm{~nm}$ CMOS process, the minimum width of the metal 1 (M1) layer is 90 $\mathrm{nm}$, which gives a minimum pitch of $180 \mathrm{~nm}$. From the above equation we could infer that such a polarizer grating would exhibit strong polarization response for wavelengths above $360 \mathrm{~nm}$. Since the visible range is roughly from $300 \mathrm{~nm}$ to $700 \mathrm{~nm}$, we can use this polarizer in the visible range for detecting the polarization state of the incoming light. 


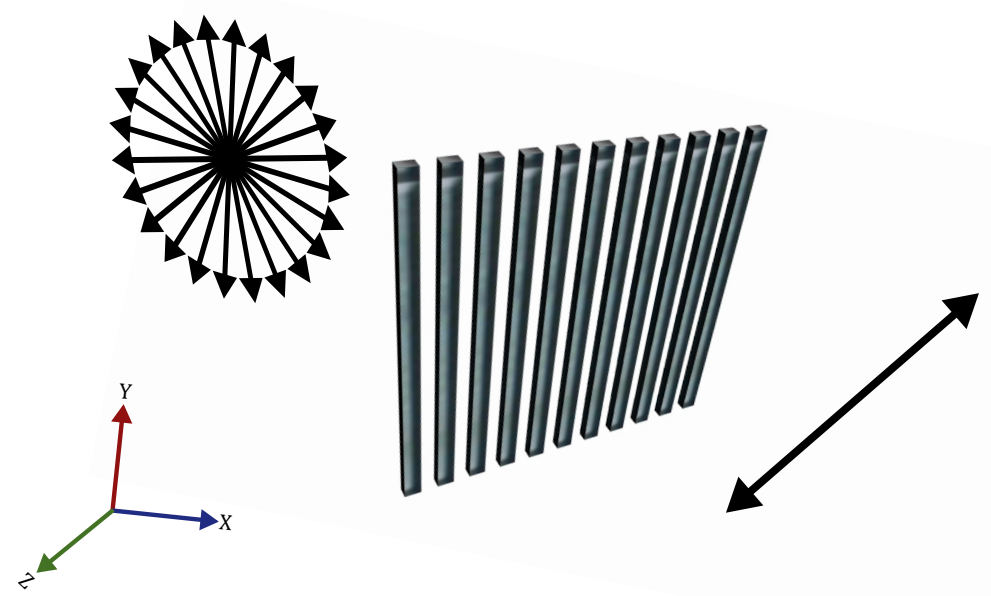

Figure 5.2: Figure showing unpolarized light being horizontally polarized by a vertical polarization grating.

Earlier works [97], [98] have shown the effect of varying the polarizer parameters and its impact on the polarization extinction ratio. For our design we have chosen to implement the polarizer grating using the metal 1 layer with a pitch of $200 \mathrm{~nm}$ and $50 \%$ duty cycle.

We make use of the angle sensitive nature of the polarizers to design a polarization pixel cluster (Fig. 5.3) with three different grating orientations for detecting incident light angles. The cluster has one pixel that is sensitive to light intensity and three pixels with 0 degree, 45 degree and 90 degree gratings that are sensitive to incident light angles and polarization. The polarizer gratings were designed using the metal 1 layer and have a width of $100 \mathrm{~nm}$ which gives a pitch of $200 \mathrm{~nm}$ with a $50 \%$ duty cycle.

Figure $5.4 \mathrm{a}$ shows the response produced by unpolarized, $90^{\circ}$ and $0^{\circ}$ polarized light when incident on a $0^{\circ}$ (horizontal) polarization pixel. As we had discussed earlier, light polarized orthogonal to the grating produces maximum response in the photodiode. In this case since the grating is $0^{\circ}, 90^{\circ}$ polarized light produces maximum response and $0^{\circ}$ polarized light produces no response. 


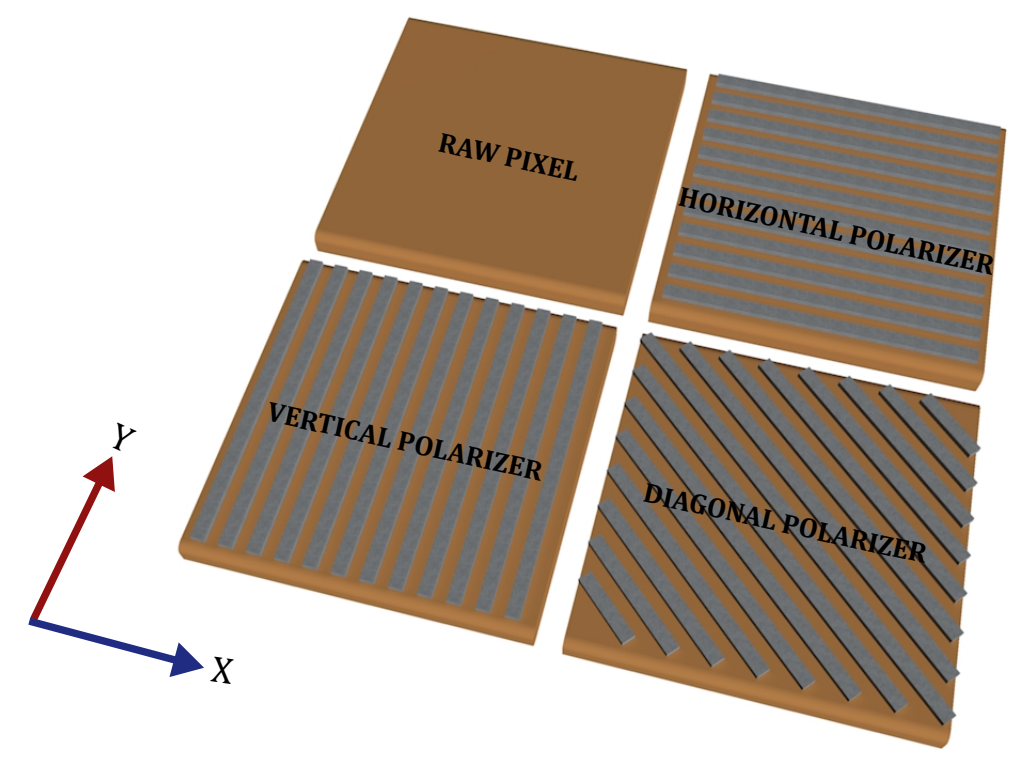

Figure 5.3: Physical structure of single-layer polarization pixels.

An alternative scenario is shown in figure $5.4 \mathrm{~b}$ in which unpolarized, $90^{\circ}$ and $0^{\circ}$ polarized light are incident on a $90^{\circ}$ (vertical) polarization pixel. As we already know by now, $0^{\circ}$ polarized light produces maximum response and $90^{\circ}$ polarized light produces no response.

The response in both the above cases were recorded by varying the incident light angle from $-45^{\circ}$ to $+45^{\circ}$ in steps of $5^{\circ}$. The response from polarization pixels show a clear cosine nature with the maximum value at $0^{\circ}$ and decreasing thereafter for oblique incidence angles.

For unpolarized light, any of the polarization pixels in the cluster will be able to detect variations in the incidence angles. On the other hand, if light is polarized we require at least two orthogonally polarized pixel gratings for angle detection. In the case of polarized light a single pixel will be sensitive to both incidence angle variation and the state of polarized light. However, if we sum the response from two orthogonal polarization pixels, we remove its polarization dependence and its sensitivity will only be proportional to angle variations.

Figure 5.5 shows the plot based on FDTD simulation for unpolarized light incident 


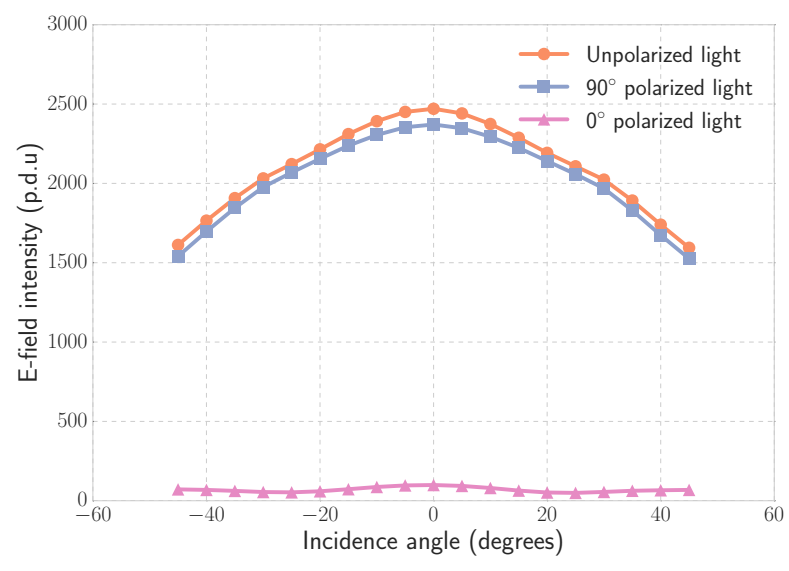

5.4.a:

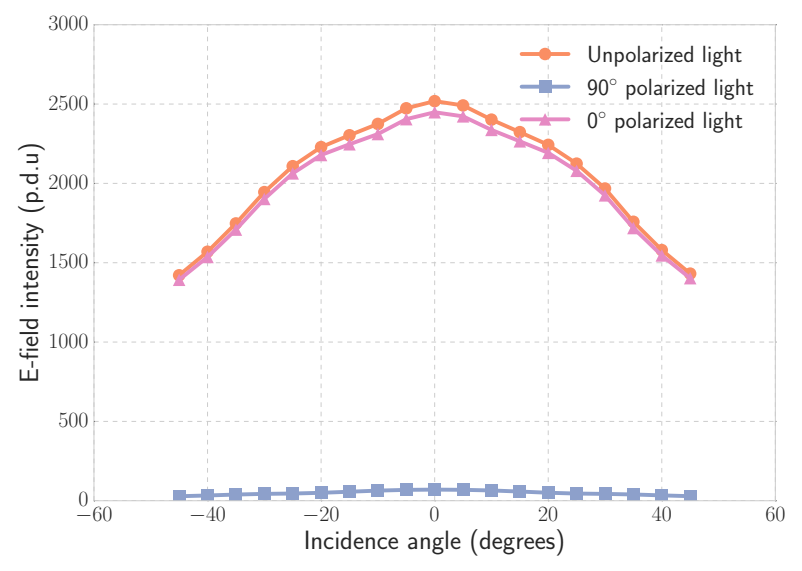

5.4.b:

Figure 5.4: Electric field intensity versus incidence angle variation for $0^{\circ}$ (a) and $90^{\circ}$ (b) polarization pixels under unpolarized light, $90^{\circ}$ or vertically polarized light and $0^{\circ}$ or horizontally polarized light.

on a $0^{\circ}$ polarization pixel and $60^{\circ}$ polarized light on $0^{\circ}$ and $90^{\circ}$ polarization pixels. When the light is polarized to $60^{\circ}$, it will have a stronger polarization component along the $\mathrm{Y}$ axis $\left(90^{\circ}\right)$ and a weaker component along the $\mathrm{X}$ axis $\left(0^{\circ}\right)$. Consequently, for a $0^{\circ}$ polarization pixel the $60^{\circ}$ polarized light produces a stronger response (because of a stronger $\mathrm{Y}$ component) and a weak response in the $90^{\circ}$ polarization pixel (because of a weaker X component). As can be seen, the summed response is same as that produced by an unpolarized light source and can be used to determine the incident angle variations independent of the polarization state of the incident light.

The polarization pixel cluster includes an additional $45^{\circ}$ grating for computing the Stoke's parameters [67] that can be used for determining the degree of polarization and the polarization angle. For the experiments presented here we only concern ourselves with the $0^{\circ}$ and $90^{\circ}$ polarization pixels, relegating an explanation for the usefulness of the $45^{\circ}$ polarization pixel to another occasion. 


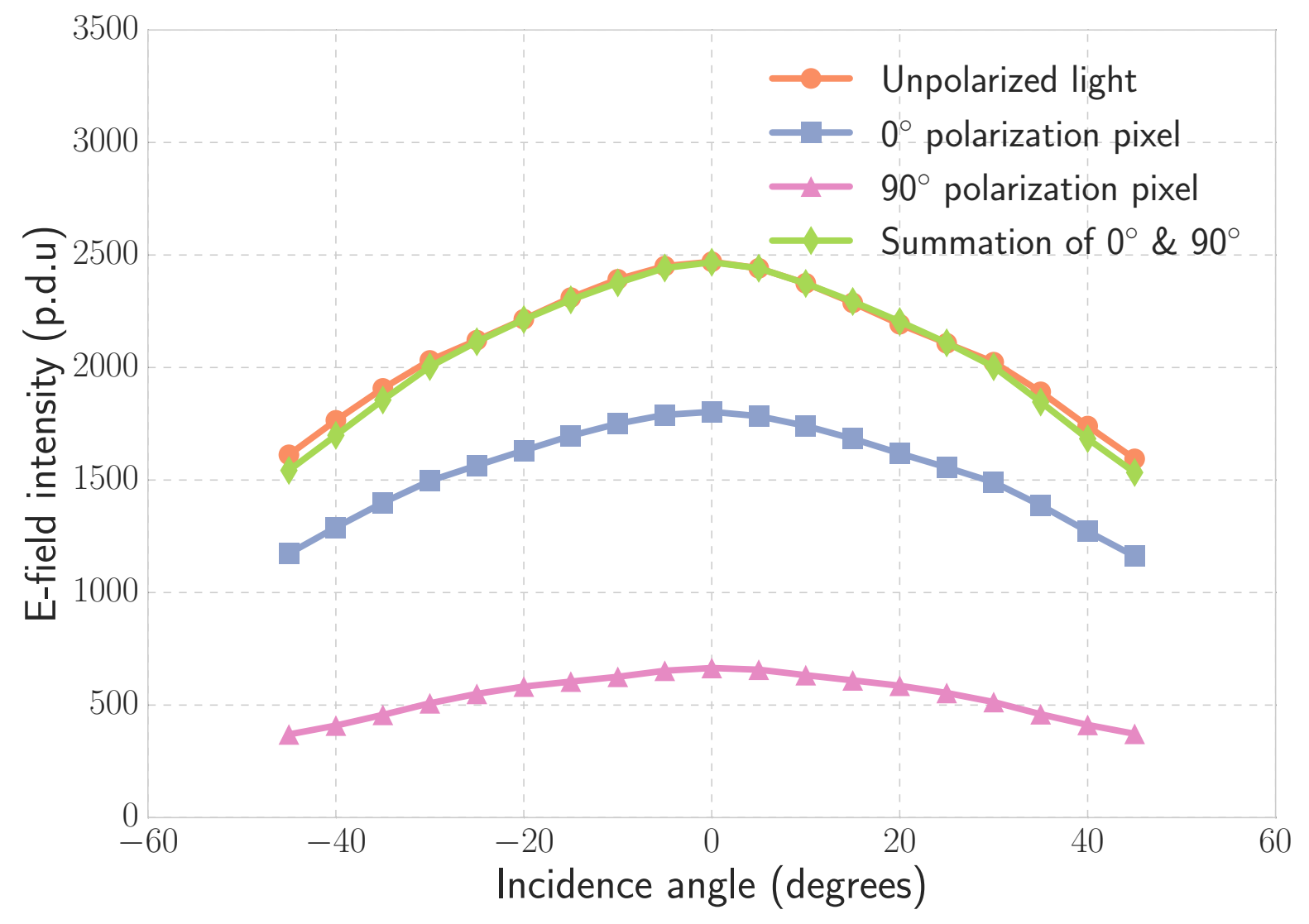

Figure 5.5: Electric field intensity versus incidence angle variation of unpolarized light on $0^{\circ}$ polarization pixel as comapred with $60^{\circ}$ polarized light on $0^{\circ}$ and $90^{\circ}$ polarization pixels along with the summed response of $0^{\circ}$ and $90^{\circ}$ polarization pixels.

\subsection{Combining Polarization Pixels with Quadrature Pixel Cluster}

It was noted earlier that by combining a high-sensitive, non-linear, periodic angular response with a low-sensitive, linear angular response we can achieve focal plane angle detection in an efficient manner using a smaller number of pixels as compared to the conventional techniques. Keeping in line with this trend, we propose another technique which combines the polarization pixel response along with the QPC difference response to determine local incidence angle with high accuracy.

As was noted in the previous section, polarization pixels respond strongly to changes 
in incidence angles, but as seen from the figures (5.4a and 5.4b) their response is inherently symmetrical around 0 degrees. That is, with just the polarization pixel response it would be impossible to determine whether the incident angle is positive or negative. This is where the QPC difference response comes into play. The QPC response is linear as was previously noted, but their sensitivity to angle change is quite small. Hence by combining the highly angle sensitive, but symmetrical polarization response with QPC response of lower sensitivity, we can efficiently determine the local angle at the pixel level. This technique consumes only a smaller number of pixels thereby effectively increasing the spatial resolution of the sensor.

The technique is illustrated in figure 5.6. In the FDTD simulation, incident light angle was varied along the horizontal direction and QPC difference response and polarization response was recorded. Positive values of the QPC response indicates negative incident angles and negative values of the QPC response indicates positive incident angles. Once we know the coarse direction of light we can use the highly angle sensitive response of the polarization pixel to accurately determine the incident angle.

Since the polarization response is strongly sensitive to incidence angle variations, changes as small as 1 or 2 degrees can be effectively measured. This is in contrast with the QPC pixels where only angles that are sufficiently far apart such as 10 degrees or even 5 degrees can be measured. By combining the two techniques, we break the inherent symmetry present in the polarization response while at the same time achieving a higher angular resolution.

\subsection{Prototype Angle Sensitive Polarization Sensor}

We designed a prototype sensor in $65 \mathrm{~nm}$ CMOS mixed-signal process to test our hypothesis. Figure 5.7 shows the micro-photograph of the sensor and gives a general idea about the sensor architecture. The sensor consists of 64 different pixel types, with the 


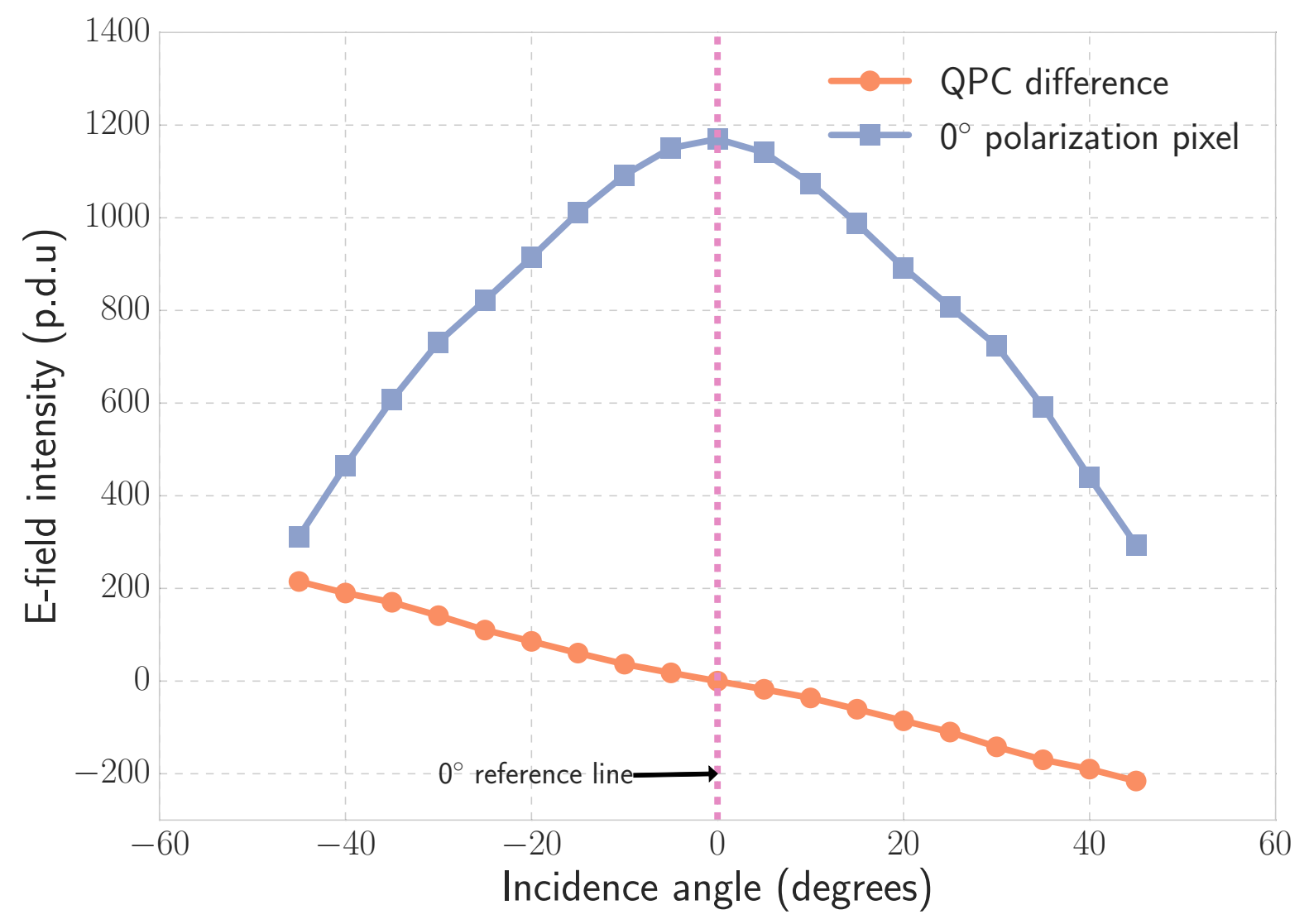

Figure 5.6: Electric field intensity versus incidence angle variation of unpolarized light on $0^{\circ}$ polarization pixel as comapred to the QPC pixel response.

main ones described in this work shown by the blow-outs. The sensor consists of the polarization cluster and the QPC cluster described earlier along with eight Talbot pixels, four for each direction (X and $\mathrm{Y}$ ) with grating offsets of $0, \mathrm{~d} / 2, \mathrm{~d} / 4$ and $3 \mathrm{~d} / 4$ which corresponds to $\alpha$ of $0, \pi, \pi / 2$ and $3 \pi / 2$ [88].

The pixels are made up of N-well/P-sub photodiode along with 3 transistors that make up a $3 \mathrm{~T}$ APS pixel. The pixel occupies an area of $16.5 \mu \mathrm{m} \times 13 \mu \mathrm{m}$ with the photodiode occupying an area of $10 \mu \mathrm{m} \times 10 \mu \mathrm{m}$ giving rise to a fill factor of $46.6 \%$. The pixel merits a large photodiode since the on-pixel metal gratings block a huge portion of light falling on the pixel from reaching the photodiode. The on-pixel gratings also prevent the use of anti-reflection coatings on top of the pixels that are common in pixels 


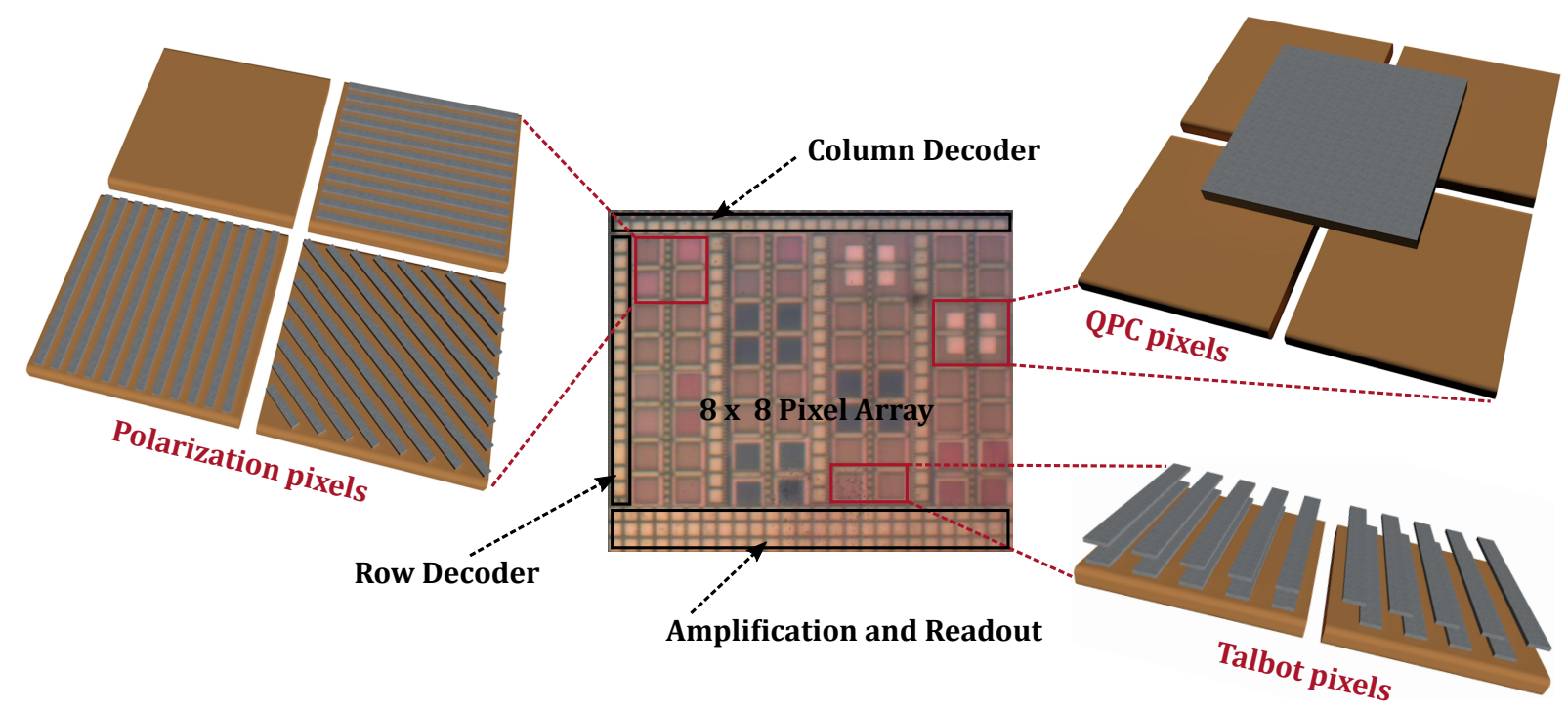

Figure 5.7: Microphotograph showing sensor architecture along with the prominent pixel types in the sensor.

fabricated using a custom image sensor process.

The polarization pixels have micro-polarizers that were implemented in metal 1 layer with a width of $100 \mathrm{~nm}$ and a pitch of $200 \mathrm{~nm}$. QPC pixels have a metal block that was implemented in metal layer 5 . The metal block covers the photodiode $6.5 \mu \mathrm{m}$ along each of the $\mathrm{X}$ and $\mathrm{Y}$ directions. The primary grating of the Talbot pixel was implemented in metal layer 5 with a pitch of $0.76 \mu \mathrm{m}$ which caused the self-images to occur at the depth of metal 1 layer. Metal 1 layer acts as the secondary grating and has the same pitch as the primary.

The pixel voltages are amplified by a factor of 2 by the global switched capacitor amplifier before being readout through a global buffer for further downstream processing. Important sensor parameters are listed in Table 5.1.

\subsection{Test Setup and Testing Methodology}

We tested the sensor for its response on incidence angle variations for multiple configurations of polarized and unpolarized light. We used the same test setup explained earlier 
Table 5.1: Important Sensor Parameters of Polarization Sensor

\begin{tabular}{|c|c|}
\hline Parameter & Value \\
\hline Technology & $65 \mathrm{~nm}$, CMOS mixed-signal process \\
Pixel size & $16.5 \mu \mathrm{m} \mathrm{x} 13 \mu \mathrm{m}$ \\
Fill factor & $46.6 \%$ \\
Pixel structure & $3 \mathrm{~T} \mathrm{APS}$ \\
Pixel power consumption & $12.13 \mu \mathrm{W}$ \\
Dark current & $26.06 \mathrm{nA} / \mathrm{cm}^{2}$ \\
Temporal noise & $1.48 \mathrm{mV}$ \\
Dynamic range & $61.77 \mathrm{~dB}$ \\
Full well capacity & $505 \mathrm{Ke}-$ \\
Conversion gain & $3.6 \mu \mathrm{V} / \mathrm{e}^{-}$ \\
Photodiode Sensitivity & $0.325 \mathrm{~V} / \mathrm{lux}-\mathrm{sec}$ \\
QPC pixel sensitivity & $5.63 \mathrm{mV} / \mathrm{deg}$ \\
Talbot pixel sensitivity & $8.70 \mathrm{mV} / \mathrm{deg}$ \\
Polarization pixel & $12.28 \mathrm{mV} / \mathrm{deg}$ \\
sensitivity & $3.3 \mathrm{~V}$ analog; $1.2 \mathrm{~V}$ digital \\
Supply voltage & \\
\hline
\end{tabular}

to test this sensor.

The raw data from the sensor is converted to its digital equivalent by an external ADC and captured by an Opal Kelly board. The data is then sent to a computer for further processing.

For experiments on incident angle variations, the rate-table was rotated in steps of 5 degrees exposing the sensor to varying angles of collimated rays from the sun simulator.

\subsection{Experimental Results}

We have extensively characterized the sensor for incident angle variations under various polarization conditions. Some of the results are presented in the following sections.

\subsubsection{Characterization of Polarization Pixels}

For characterizing the polarization pixels we varied the incident light angle from $-45^{\circ}$ to $+45^{\circ}$ in steps of $5^{\circ}$ and measured the recorded response. 
Figure 5.8a shows the response produced by a $0^{\circ}$ polarization pixel for unpolarized, $90^{\circ}$ polarized and $0^{\circ}$ polarized light. As we had observed with the FDTD simulations, the response of the pixel for light polarized orthogonal $\left(90^{\circ}\right.$ polarized light) to the grating is maximum. While light polarized parallel $\left(0^{\circ}\right.$ polarized light $)$ to the grating is minimum.

Similarly figure $5.8 \mathrm{~b}$ shows the response produced by a $90^{\circ}$ polarization pixel for unpolarized, $90^{\circ}$ polarized and $0^{\circ}$ polarized light. In this case again response for light polarized orthogonal $\left(0^{\circ}\right.$ polarized light $)$ to the grating is maximum. While light polarized parallel $\left(90^{\circ}\right.$ polarized light) to the grating is minimum.

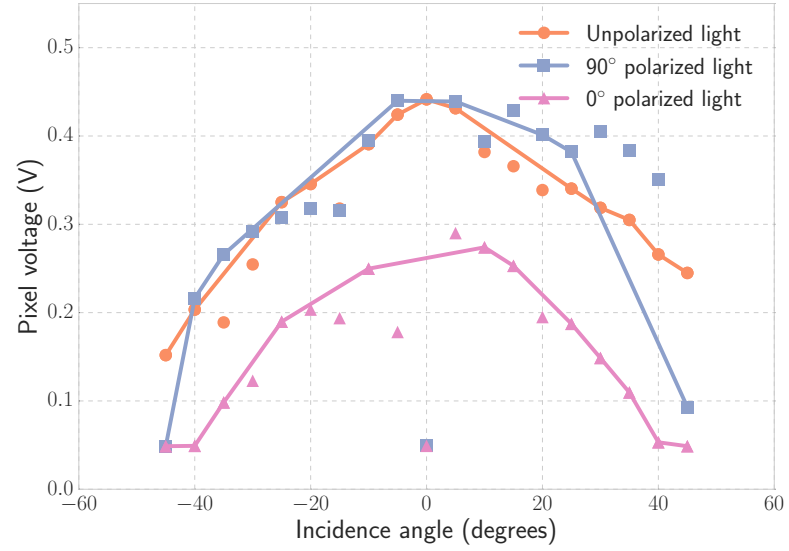

5.8.a:

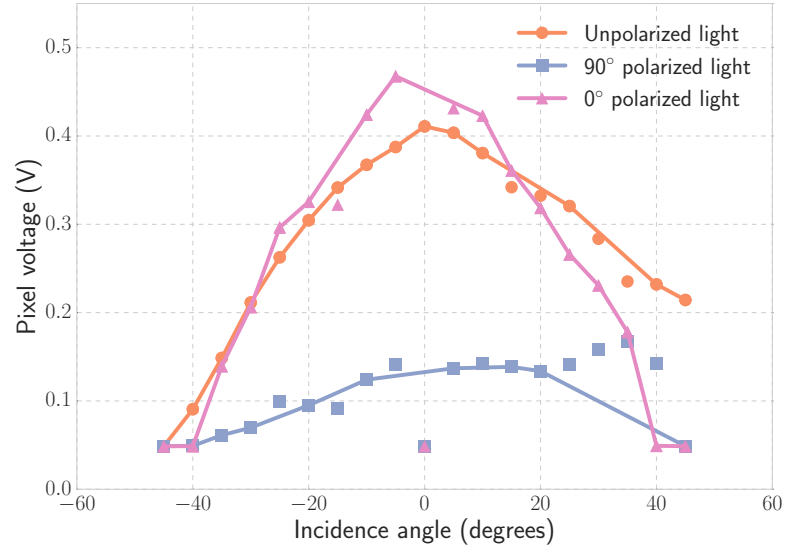

5.8.b:

Figure 5.8: Pixel voltage versus incidence angle variation for $0^{\circ}$ (a)and $90^{\circ}$ (b) polarization pixels under unpolarized light, $90^{\circ}$ or vertically polarized light and $0^{\circ}$ or horizontally polarized light.

Figures 5.9 and 5.10 reinterpret the above results in a slightly different manner for better clarity. Figure 5.9a shows the response produced by $0^{\circ}$ polarized light when incident on $0^{\circ}$ and $90^{\circ}$ polarization pixels. For a $0^{\circ}$ polarized light, $0^{\circ}$ polarization pixel produces minimum response whereas $90^{\circ}$ polarization pixel produces maximum response.

In a similar manner figure $5.9 \mathrm{~b}$ shows the response produced by $90^{\circ}$ polarized light when incident on $0^{\circ}$ and $90^{\circ}$ polarization pixels. For $90^{\circ}$ polarized light, $0^{\circ}$ polarization 
pixel produces maximum response whereas $90^{\circ}$ polarization pixel produces minimum response.

Figure 5.10 shows the response produced by unpolarized light when incident on $0^{\circ}$ and $90^{\circ}$ polarization pixels, which is in agreement with the theory that the response produced by polarization gratings of random orientations to unpolarized light is equal.

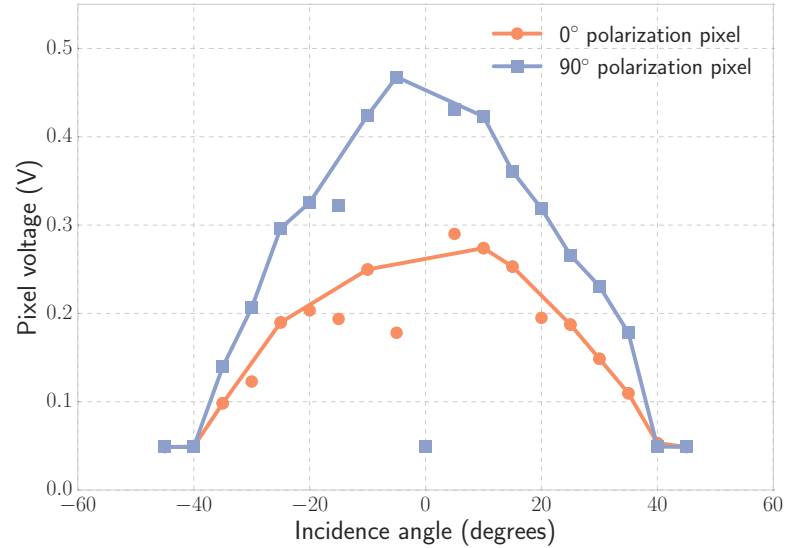

5.9.a:

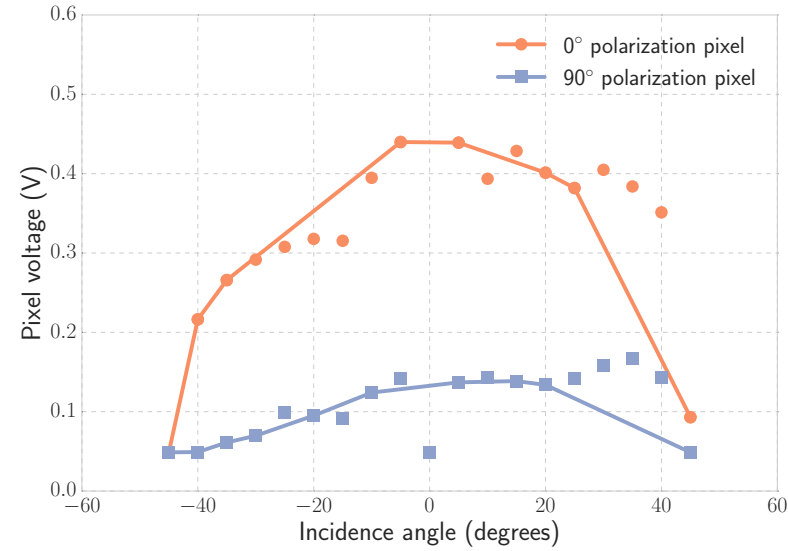

5.9.b:

Figure 5.9: Pixel voltage versus incidence angle variation for $0^{\circ}$ and $90^{\circ}$ polarization pixels under $90^{\circ}$ or vertically polarized light (a) and $0^{\circ}$ or horizontally polarized light (b).

\subsubsection{Comparing Polarization, Talbot and QPC Pixel responses}

Figure 5.11 contrasts the response produced by Polarization, Talbot and QPC pixels. The responses were recored by varying incident light angle horizontally (along $\mathrm{X}$ axis) from $-45^{\circ}$ to $+45^{\circ}$ in steps of $5^{\circ}$. We have used a $90^{\circ}$ polarization pixel with $0^{\circ}$ polarized light for comparison.

As expected the difference response from the QPC pixels (along X direction) is linear. The response from polarization pixel has a cosine nature and that from the Talbot pixel is periodic, with its periodicity dependent on the grating parameters.

As seen from the plot, the angle sensitivity of the polarization and Talbot pixels are higher than the QPC pixels. The sensitivity of an angle sensitive pixel can be defined as 
the response produced by the pixel for one degree change in incident light angle. It can be expressed in $\mathrm{mV} / \mathrm{deg}$. Table I gives the angle sensitivities for each of the pixel types.

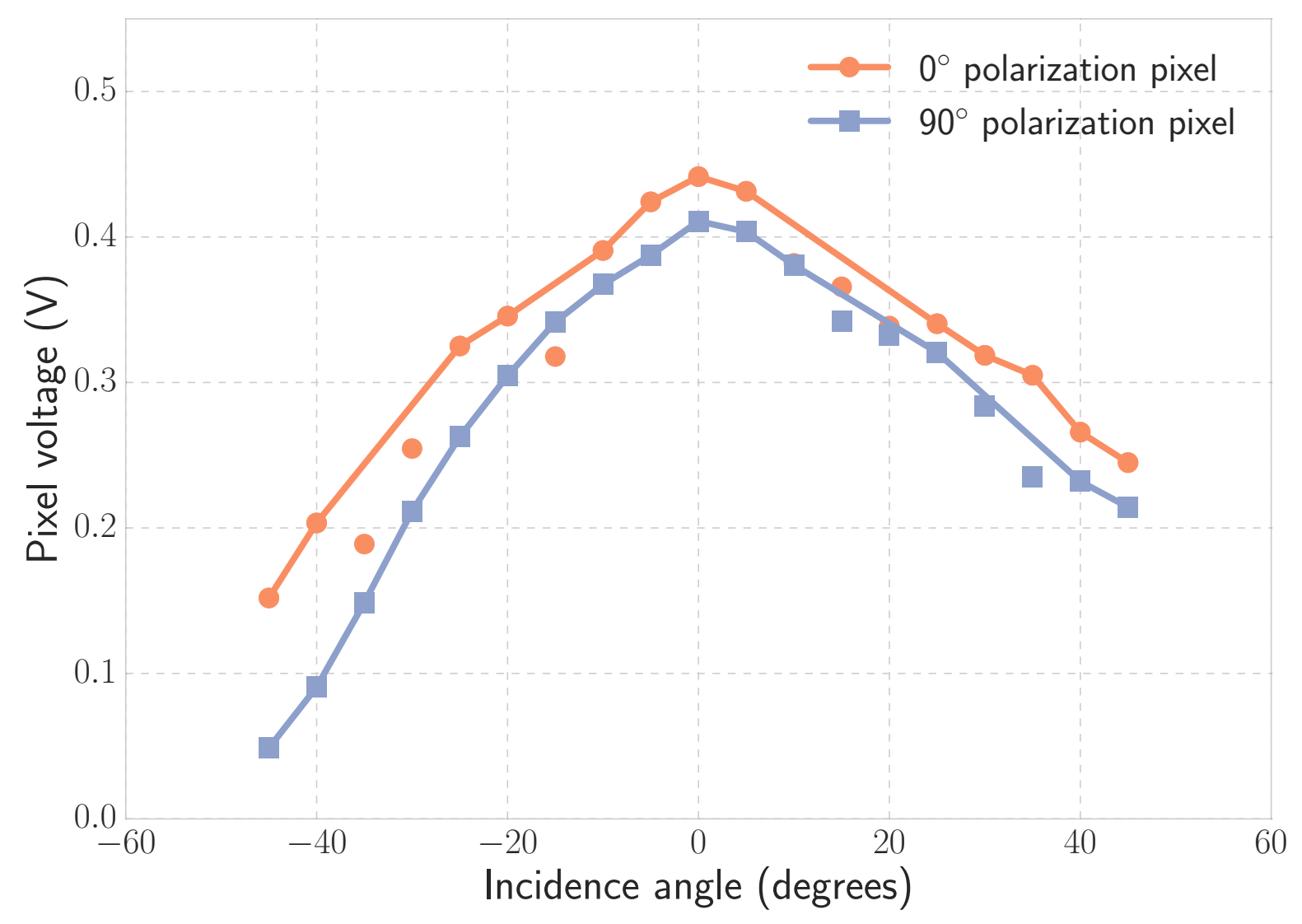

Figure 5.10: Pixel voltage versus incidence angle variation for $0^{\circ}$ and $90^{\circ}$ polarization pixels under unpolarized light.

The periodic nature of the Talbot response requires multiple angle-uwrappings with aid from the QPC response in order to decode the incident angle. Polarization response on the other hand is a simple cosine curve with a peak at $0^{\circ}$ and requires just one angle-unwrappping to uniquely decode the incident light angle.

\subsubsection{Angle Detection using Polarization and QPC Pixels}

The principle of angle detection using polarization and QPC pixel was introduced in section III. We illustrate the same here with experimental results. Figure 5.12 shows the 


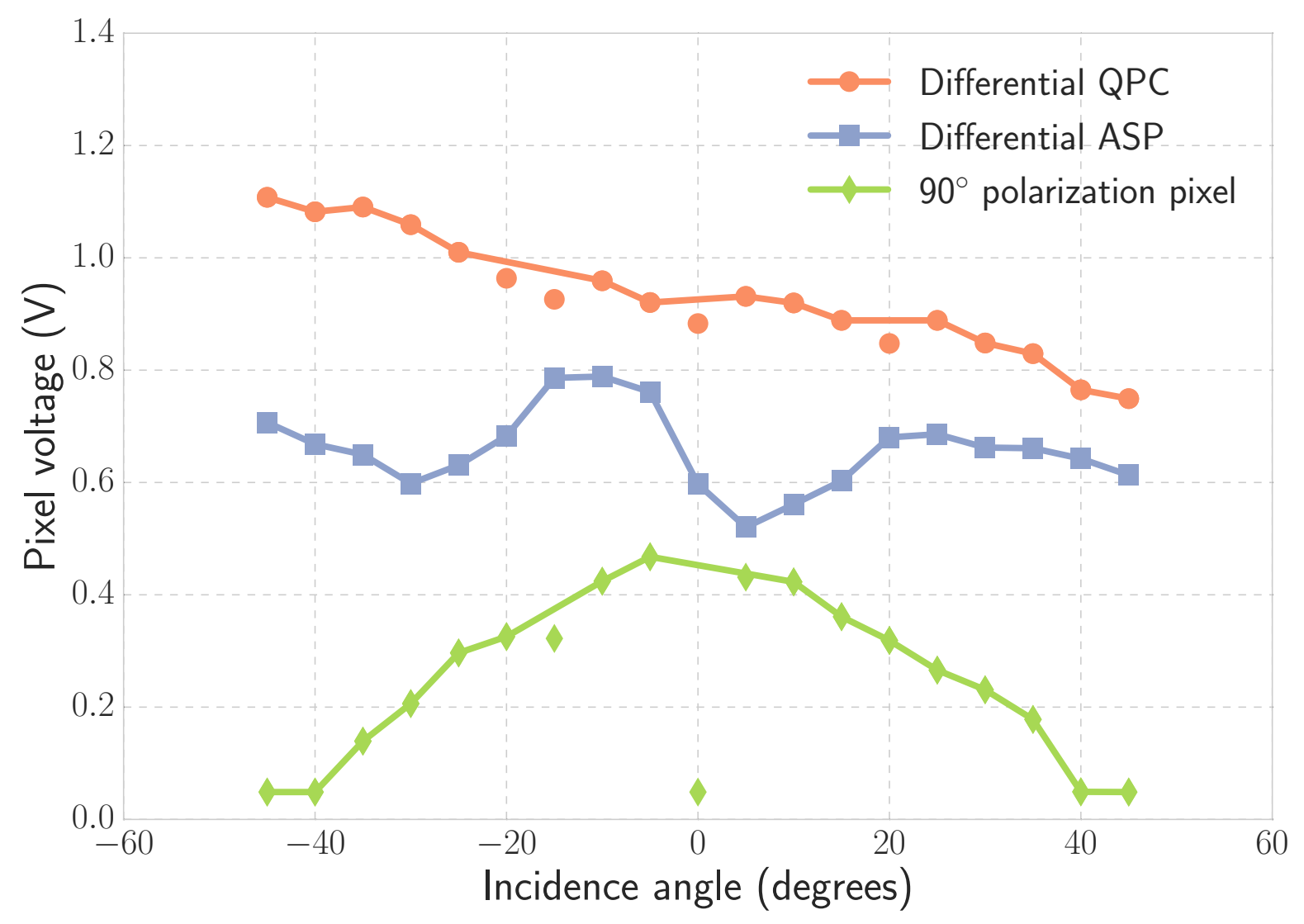

Figure 5.11: Pixel voltage versus incidence angle variation of differential quadrature pixel cluster (QPC), differential Talbot effect based angle sensitve pixel (ASP) and 90 polarization pixel.

polarization pixel response and the QPC pixel response from the previous subsection. As seen from the figure positive voltages of the QPC response indicates that the incident angle is negative and negative voltages of the QPC response indicates that the incident angle is positive.

Once we know the sign of the incident angle (positive or negative) we can use the corresponding half of the polarization response to get an accurate value of the incident light angle. 


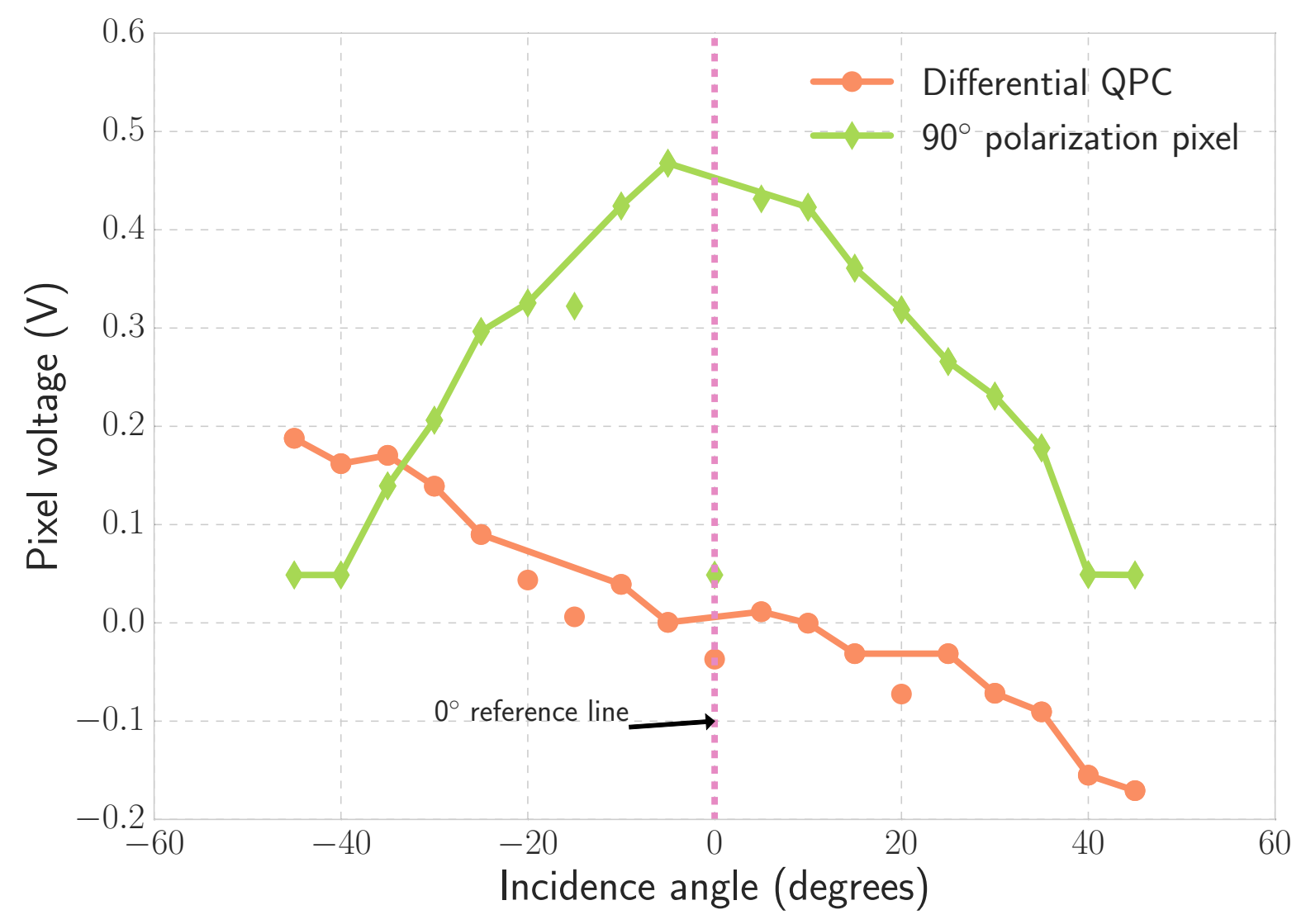

Figure 5.12: Pixel voltage versus incidence angle variation of differential quadrature pixel cluster (QPC) and $90^{\circ}$ polarization pixel illustrating the angle detection technique.

\subsection{Discussion}

Several factors contribute to the nonidealities in the results obtained from the designed prototype sensors. Some factors are a result of the pixel design and others are a result of the limitations in the experimental setup. We discuss some of these factors here.

\subsubsection{Design Limitations}

Design limitations are either due to the restrictions in designing an image sensor pixel or the inherent limitations in the CMOS fabrication process.

(i) Optical Pixel Crosstalk: Optical Pixel crosstalk [10] results when light meant 
for one pixel falls on its adjacent neighbor thereby producing unwanted response. For large positive and negative angles crosstalk could become a serious issue which increases the angle insensitive baseline pixel response. This in fact reduces the per-degree angle sensitivity of a particular pixel. This could be one of the issues that contribute to the less than ideal characteristic of the pixel response at large positive or negative angles.

(ii) Pixel Vignetting: Pixel vignetting [13] is another factor that contributes to the angle dependent nature of a pixel and is a result of the interconnect metal layers in the vicinity of the photodiode. Typically, pixel vignetting is influenced more by the light-shield around the photodiode that is used to enforce uniformity along all the photodiode directions to incident light rays.

For normal incidence, there is no shadow on the photodiode because of the light shield and the photodiode produces maximum response. On the other hand, for oblique angles the response reduces as a function of the incident light angle.

The above limitations are a result of the pixel architecture and are difficult to eliminate under normal circumstances. For example, in order to reduce pixel crosstalk, the pixels have to be placed very far apart, which in fact is not practical as it increases the sensor area without increasing the sensor pixel resolution. Similarly pixel vignetting can be reduced by eliminating any metal layers in the vicinity of the photodiode. This again is not practical as it would require a large pixel size and is not feasible because of the above mentioned reason.

\subsubsection{Experimental Limitations}

These limitations arise because of the inefficiencies in the test setup and testing methodology. 
(i) Lambert's Cosine Law: Lambert's cosine law states that the light incident on a surface with a fixed area at an oblique angle of incidence is equal to the cosine of its value at normal incidence. This is given as $\mathrm{I}(\theta)=\mathrm{I}(0) \operatorname{Cos}(\theta)$. The consequence of this law is that, when the light source is wider than the pixel and the incident angle is $\theta$, which is not $0^{\circ}$, the optical power on the surface of the photodiode decreases as the angle increases. This introduces an angle dependent behavior for a conventional intensity pixel. The effect of just the cosine law on the angle sensitivity of a pixel is very weak and is a non-issue when a lens is introduced to focus the light beam, unlike in the present scenario.

(ii) Sensor-Light Source Alignment: Misalignment between the sensor and the sun-simulator (light source) will introduce a slight angle dependence to the recorded responses.

(iii) Control of Angle Variation: The angle made by the sensor with the light source was varied by letting the rotary table rotate at a fixed rate for a particular amount of time. Even though the rotation was controlled by a PC, latencies in the instruction execution pipeline adversely impacted the angle variation of the rotary table.

(iv) Ray Divergence: The collimated light ray from the sun simulator diverges from its normal angle at distances away from the sun simulator. The amount of divergence depends on the actual distance between the sensor and the sun simulator and could have been a small contributing factor to inaccurate angle measurements.

(v) Temperature Effects: At small distances from the sun simulator, the ambient temperature increases to a non-negligible amount. Prolonged operation of the sun simulator results in a sharp increase of the sensor dark current. 
(vi) Unwanted Polarizaton of Light: The sun simulator has a glass-covering around its outer edge and it contributed to a slight polarization of the incident light. Hence light from the sun simulator was partially polarized with a small horizontal polarization component instead of being completely unpolarized. 


\section{Chapter 6}

\section{Angle Sensitive Imaging: Evaluation and Applications}

In the first part of this chapter we compare and contrast the various angle sensitive and light field image capturing techniques to understand the underlying trade-offs one grapples with when trying to choose a suitable solution to a particular problem. In the final part of this chapter we demonstrate three applications facilitated by the angle sensitive imaging techniques.

\subsection{Framework for Evaluating Angle Sensitive Imag- ing}

The past few chapters introduced three major techniques for angle sensitive imaging. A common thread than ran through those chapters were the increase in the spatial resolution as a result of decreasing the number of pixels needed for angle detection. Some of the techniques also offer distinct advantages, such as the polarization pixels that capture both polarization as well as incident light angle information. Yet others, such as the orthogonal sinusoidal MF pixels, sacrifice angular range to increase the spatial resolution. This section makes a comparative analysis of all the angle sensitive pixels that were presented in this thesis together with the diffraction based techniques (Talbot 
imaging and enhanced Talbot imaging) and the plenoptic techniques (plenoptic imaging and focused plenoptic imaging).

The approaches for angle detection presented in this thesis so far have complex trade-offs when it comes to angle detection. In the sections that follow we will evaluate the plenoptic [59], focused plenoptic [24], Talbot [88], enhanced Talbot [71], trackand-tune [84], polarization [82], antisymmetric multi-finger [81] and orthogonal multifinger [79] sensors in terms of spatial resolution, angular resolution, light transmittance, angle sensitivity, wavelength and polarization sensitivity to characterize their usefulness.

The use of a particular kind of pixel structure depends on the intended application, available fabrication process and intended complexity of algorithms.

The spatial and angular resolution defined below should not be confused with that of the one related to the Raleyigh's criterion for a diffraction limited system.

\subsubsection{Spatial Resolution}

Spatial resolution for a light field image sensor is set by the number of angle sensitive pixels that capture the local angle information. The plenoptic camera trades off spatial resolution for angular resolution. The number of microlenses decide the spatial resolution, whereas the number of pixels underneath each microlens determines the angular

resolution. For a sensor with $\mathrm{N}$ pixels, the spatial resolution of the plenoptic camera would be N/16 (for 16 pixels underneath each microlens).

The focused plenoptic camera sacrifices angular resolution for obtaining higher spatial resolution. Based on [24] we can estimate that there is a loss of spatial resolution by a factor of around 7. Thus the effective spatial resolution is $\mathrm{N} / 7$ for a $\mathrm{N}$ pixel sensor.

The Talbot sensor [88] uses 64 distinct angle sensitive pixels for light angle detection. Apart from accounting for the directional dependence of gratings (4 different directions) these pixels also take care of angular dependence of the light source with respect to the 
gratings (since the secondary grating can block light at some specific angles). Overall, they need 64 pixels for appreciable angular resolution. For a Talbot sensor with N pixels, the achievable spatial resolution is only $\mathrm{N} / 64$.

At this point it is important to clarify the in-focus spatial resolution and the out-offocus spatial resolution. In-focus spatial resolution is the resolution of the sensor when the scene is in perfect optical focus. For a Talbot sensor, the in-focus spatial resolution would degrade only by a factor of 2 . That is the overall degradation in spatial resolution would be $\mathrm{N} / 2$. However when the scene is out of focus, the intensity information is of little importance as compared to the angular information. The N/64 spatial degradation that was mentioned earlier for the Talbot sensor is for out-of-focus images. The resolution degradation that we mention in this section is for out-of-focus images. This is in contrast to the plenoptic techniques that have a fixed degradation (N/16 for plenoptic camera and $\mathrm{N} / 7$ for focused plenoptic camera) irrespective of whether the scene is in-focus or out-of-focus.

The enhanced Talbot sensor is based on the same principle as the Talbot sensor and thus requires the same number of pixels for local angle detection (64). The spatial resolution is again reduced by a factor of $64(\mathrm{~N} / 64)$ for an array with $\mathrm{N}$ pixels.

The track-and-tune sensor uses a combination of the Tablot pixels and QPC pixels for angle detection. This sensor uses 13 pixels for local angle detection which reduces the spatial resolution by $\mathrm{N} / 13$ for a $\mathrm{N}$ pixel sensor.

The polarization sensor works on the principle of polarization and uses up 8 pixels for local angle detection leading to a reduction in spatial resolution by a factor of 8 . Thus a $\mathrm{N}$ pixel sensor will have a spatial resolution of $\mathrm{N} / 8$.

The antisymmetric multi-finger sensor uses 4 pixels for local angle detection leading to a reduction in sensor resolution by a factor of 4 . Thus a $\mathrm{N}$ pixel sensor will have a spatial resolution of $\mathrm{N} / 4$. 


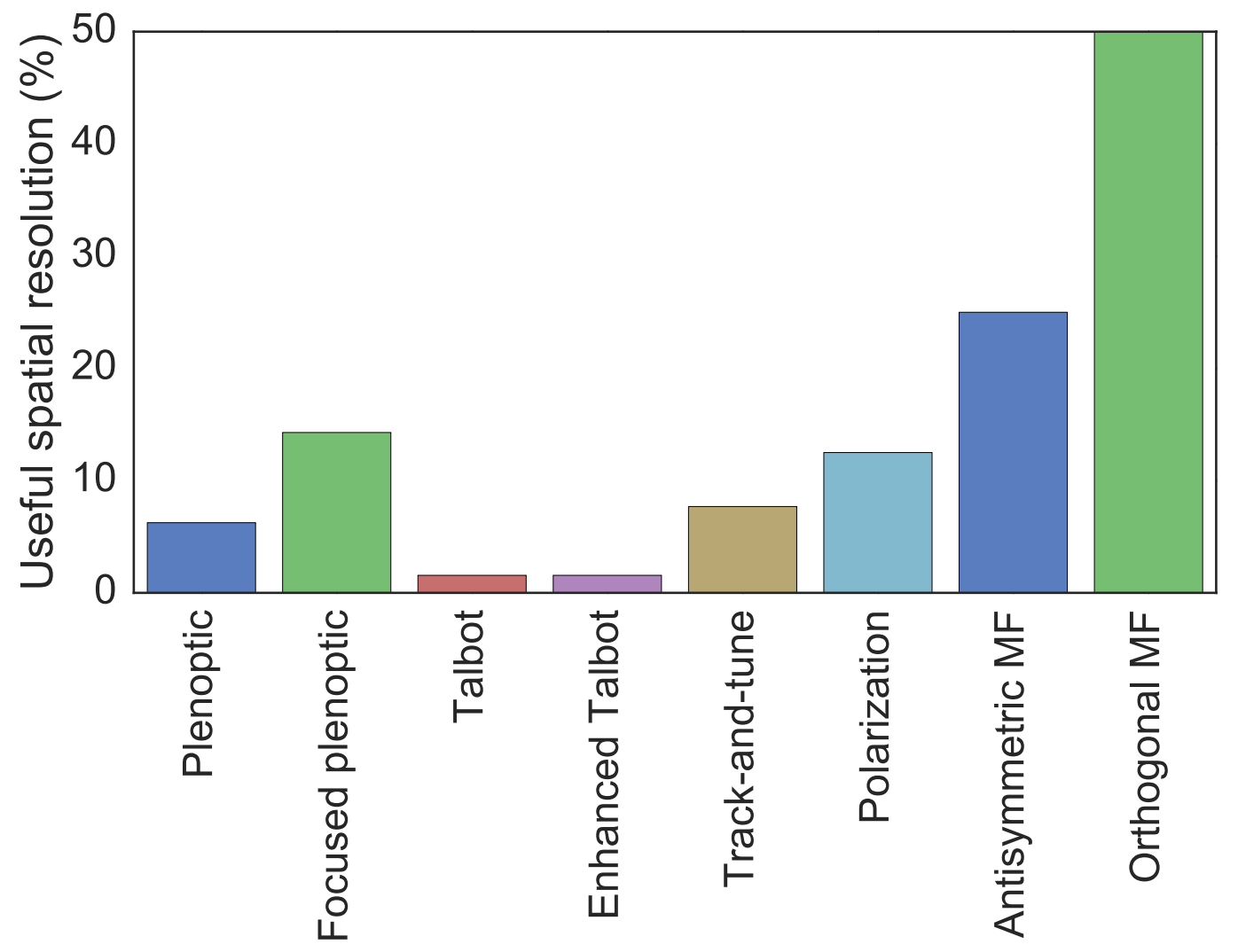

Figure 6.1: Spatial resolution of various light field imaging techniques.

The orthogonal multi-finger sensor takes this approach a notch higher by being able to detect local angles with a reduction in spatial resolution by a factor of 2 . Thus a $\mathrm{N}$ pixel sensor will have a spatial resolution of $\mathrm{N} / 2$.

Figure 6.1 plots the effective spatial resolution for the above mentioned light field imagers. For a $\mathrm{N}$ pixel sensor, the loss in spatial resolution will be $\mathrm{N} / \mathrm{L}_{s}$, where $\mathrm{L}_{s}$ is the spatial resolution loss factor (e.g., 16 for plenoptic camera). We plot the inverse of the loss factor $1 / \mathrm{L}_{s}$, as a percentage, which tells us the amount of useful pixels in the sensor. 


\subsubsection{Angular Resolution}

We define the angular resolution of a light field sensor as the range of unique incident light angles that the sensor is capable of determining at a macro-pixel level in the absence of any external optics (including the objective lens). In the presence of a lens and aperture, this value will be limited by the sensors' field of view. For plenoptic cameras this is equal to the field of view of the sensor and is determined by the lens aperture and sensor size.

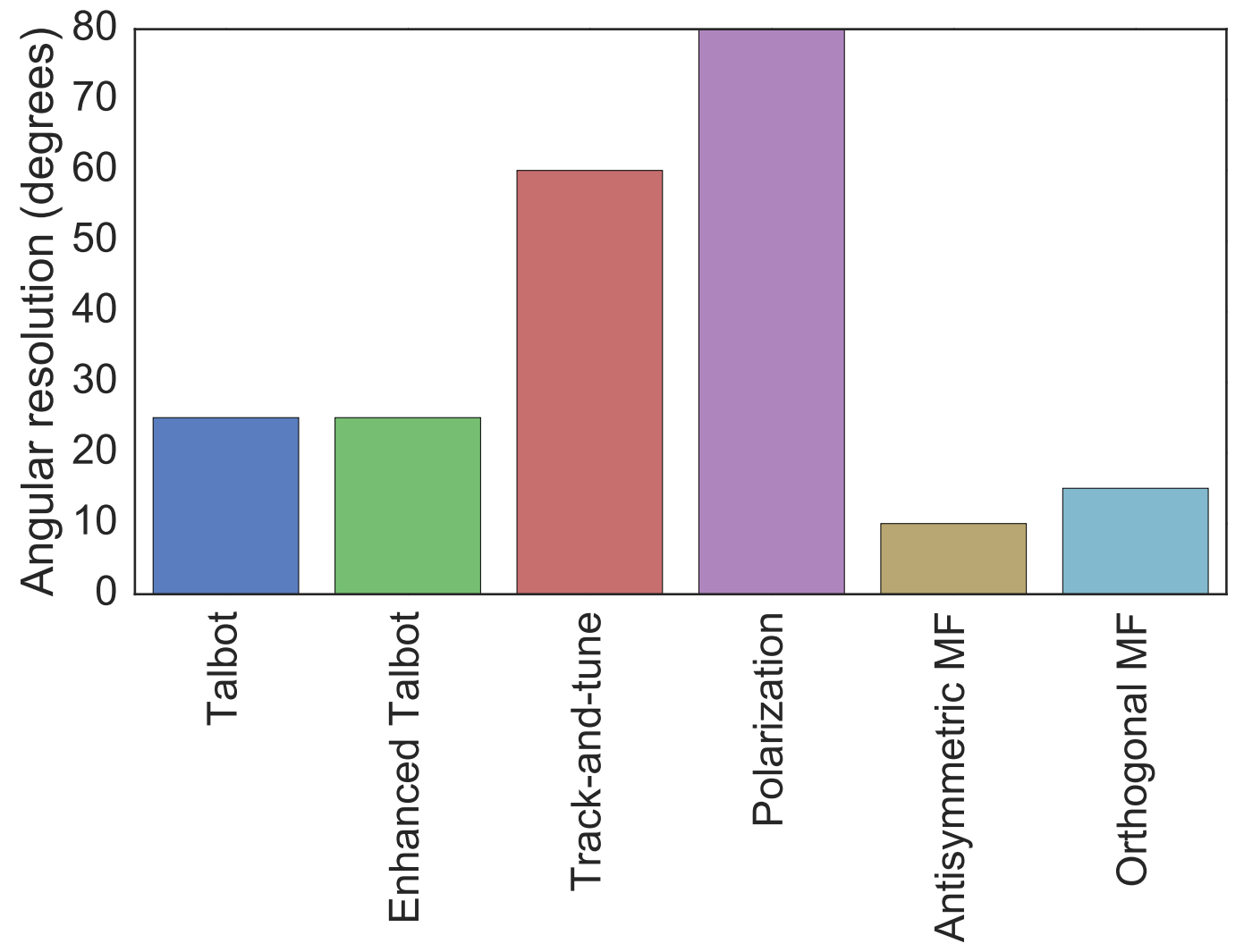

Figure 6.2: Angular resolution of various angle sensitive pixels.

For Talbot and enhanced Talbot sensors angular resolution is the total range of unique angles along a particular orientation that pixels with various $\alpha$ and $\beta$ values can detect. The angular resolution for this sensor comes to around $25^{\circ}$ (considering the curves for $\beta=7.6[88])$. 
For the track-and-tune sensor, the angular range is wider due to the QPC pixels. The angular resolution for this sensor turns out to be roughly around $60^{\circ}$ [84] [82].

For polarization sensor the angular resolution turns out to be roughly around $80^{\circ}[82]$.

For antisymmetric MF sensor the angular resolution turns out to be around $10^{\circ}$ [81] and for orthogonal MF sensor it turns out to be around $15^{\circ}$ [79]. For the fabricated orthogonal $\mathrm{MF}$ sensor this value is around $25^{\circ}$ to $30^{\circ}$, due to light impinging on the walls of the N-well/P-sub photodiodes, which the FDTD simulation did not account for. However, for the purpose of fair comparison we use the simulated values as this will keep the baseline same for all the pixel types (from all the different sensors).

\subsubsection{Light Transmittance}

Since angle sensitive pixels employ micron scale metallic structures at the image sensor focal plane to evoke angle sensitive behavior, the light transmitted onto the surface of the photodiode is lower than that of a conventional pixel. This introduces challenges for low light and high speed imaging. For very low light transmittance, one might have to resort to pixel readout with high gain to create a visible image, which leads to grainier images. For static scenes one might use longer exposure, which requires complex optical stabilization techniques to counter the effects of hand-shake. It also makes it infeasible to image scenes with moving components. Figure 6.3 shows light transmittance for various angle sensitive pixels. Light transmittance is defined as the ratio of light transmitted to the photodiode to the light incident on the pixel (before the metal gratings and masks).

For all pixels light transmittance was taken at the angle that produced maximum response. Talbot pixels with $\alpha=0$ were considered. For enhanced Talbot pixels we took the data from [71] and interpolated the result with respect to the Talbot pixels and used this value for the plot. The light transmittance is just one side of the coin. These results should always be considered together with the angle sensitivity of these pixels. 


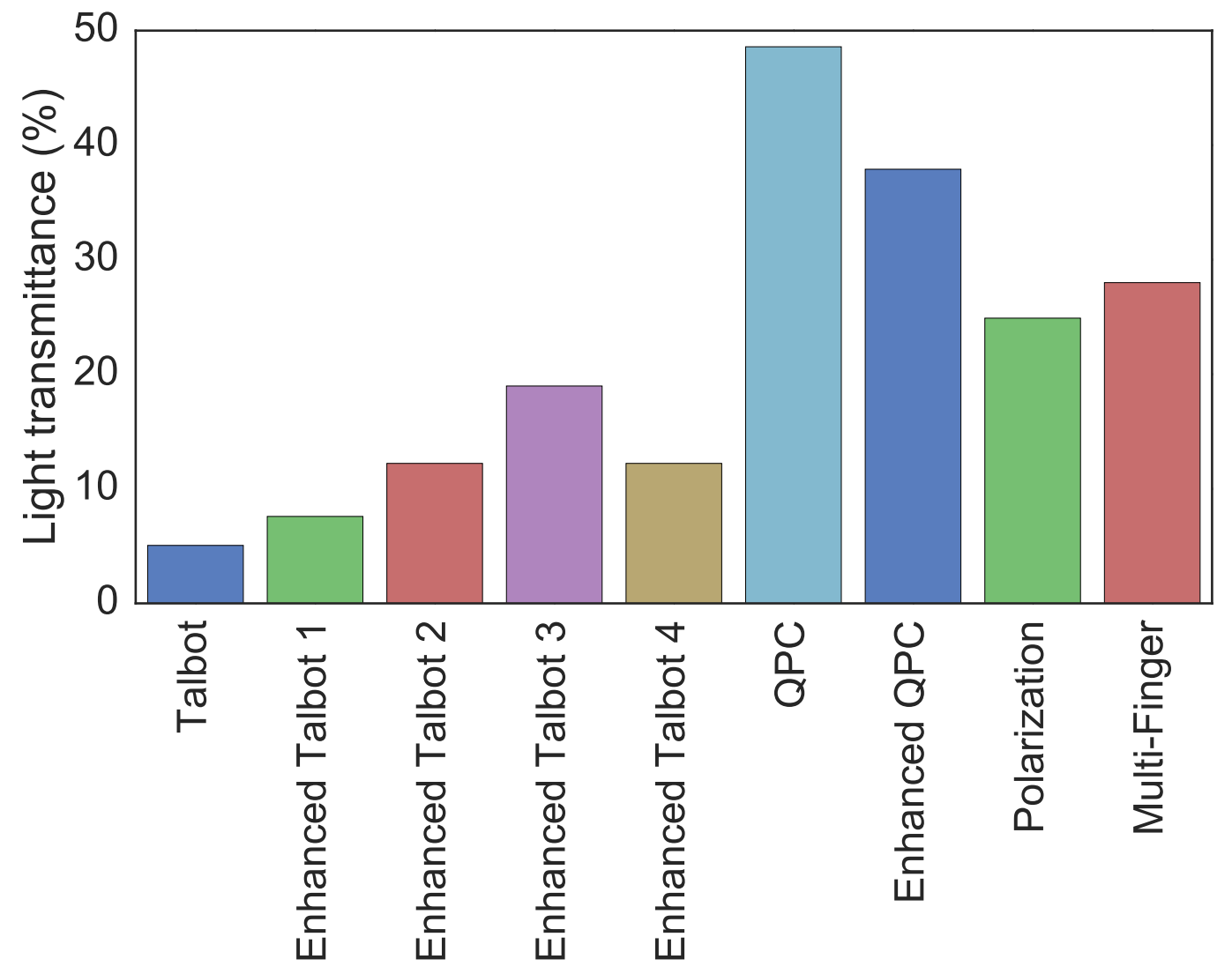

Figure 6.3: Light transmittance for various angle sensitive imaging pixels. 'Enhanced Talbot 1' uses amplitude grating with interleaved N+/P-sub diode, 'Enhanced Talbot 2' uses phase grating with N-well/P-sub diode, 'Enhanced Talbot 3' uses phase grating with interleaved N+/P-sub diode, 'Enhanced Talbot 4' uses amplitude grating with interleaved $\mathrm{P}+/ \mathrm{N}$-well diode.

The 'Enhanced Talbot 1', 'Enhanced Talbot 2', 'Enhanced Talbot 3', 'Enhanced Talbot 4' are the enhanced Talbot pixels as described in section 3.4.

\subsubsection{Angle Sensitivity}

Angle sensitivity is defined as the change in pixel response (in milli Volts) as a result of $1^{\circ}$ change in incident light angle. Figure 6.4 plots the angle sensitivity for the various pixel types. Since we did not fabricate a antisymmetric multi-finger sensor, its sensitivity 
was approximated to be twice as that of the orthogonal multi-finger pixel.

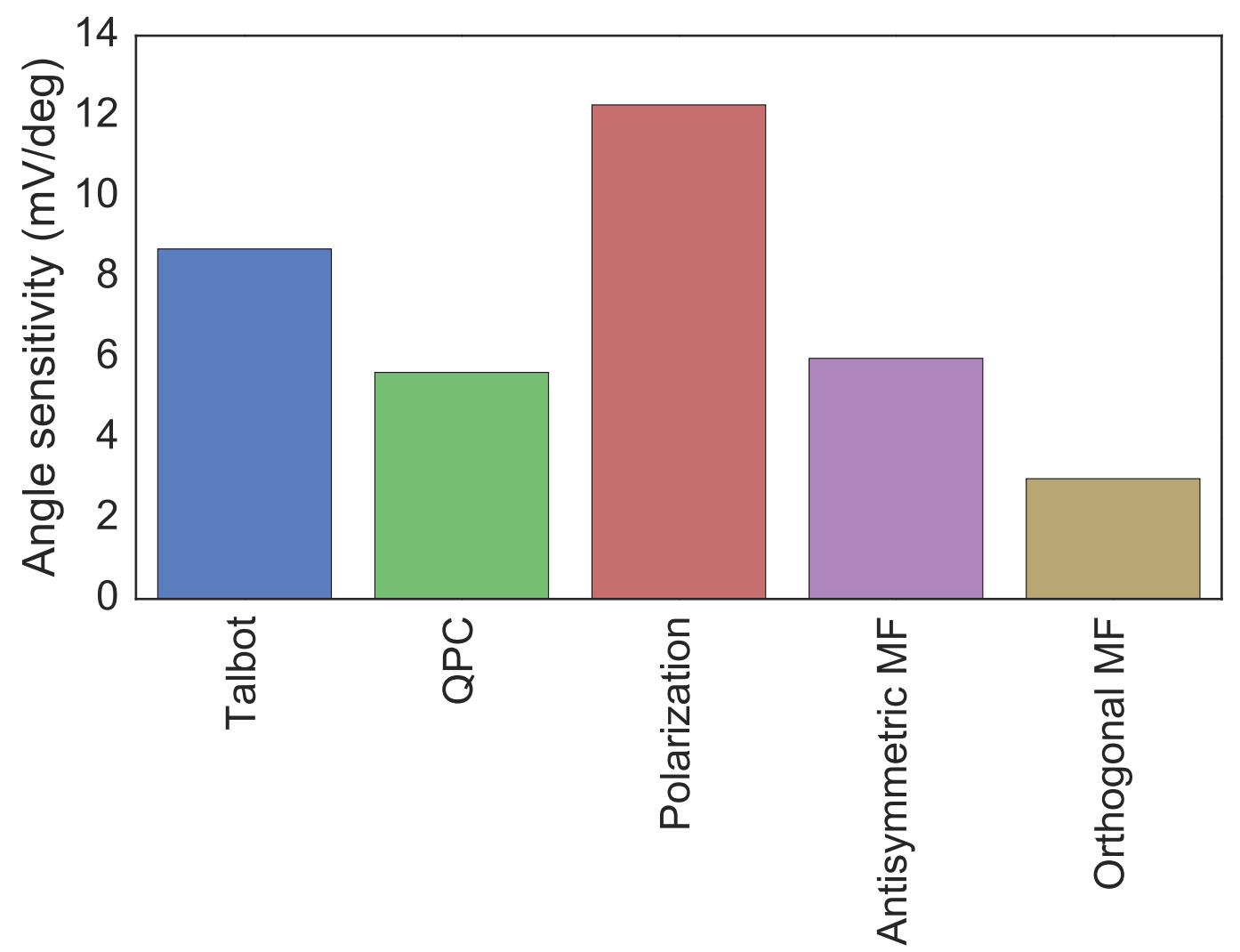

Figure 6.4: Angle sensitivity of various angle sensitive imaging pixels.

\subsubsection{Wavelength Sensitivity}

In this section we examine the response of various angle sensitive pixels to wavelength changes. Since we use wavelength scale gratings and masks on top of the pixels, it is interesting to see if the pixel behavior changes with wavelength variations.

Figure 6.5(a) shows the response produced by a Talbot pixel as the wavelength is changed from $400 \mathrm{~nm}$ to $600 \mathrm{~nm}$. We see the peak response shifting and is expected as the Talbot depth, that is the basis of this pixel type, shifts as a result of wavelength shifts. The shift can be characterized by the equation given below. 


$$
\mathrm{z}_{\mathrm{t}}=\frac{2 \mathrm{~d}^{2}}{\lambda}
$$

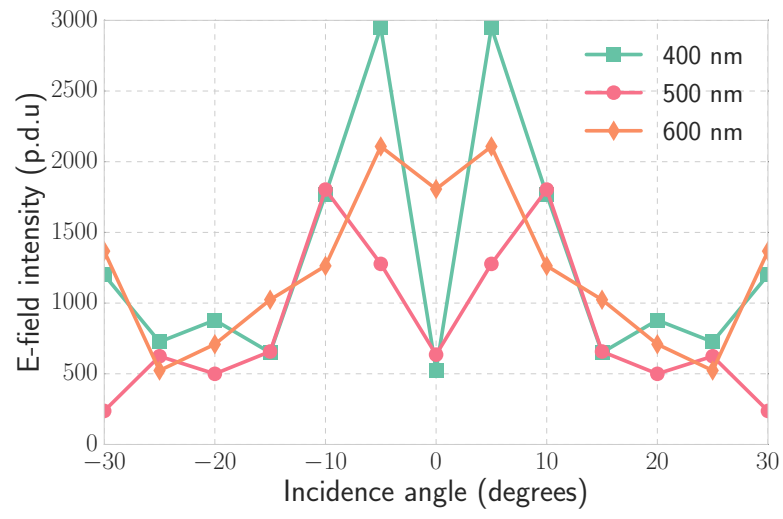

6.5.a: Response of Talbot $(\alpha=0)$ pixels.

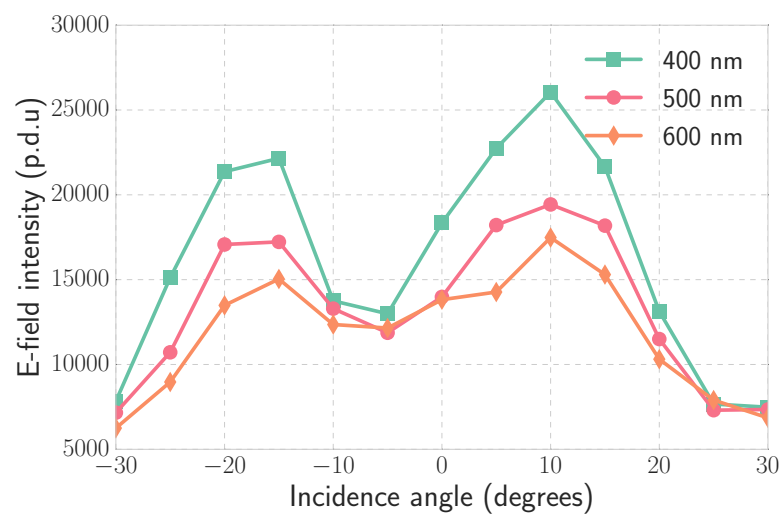

6.5.c: Response of horizontal multi-finger pixel.

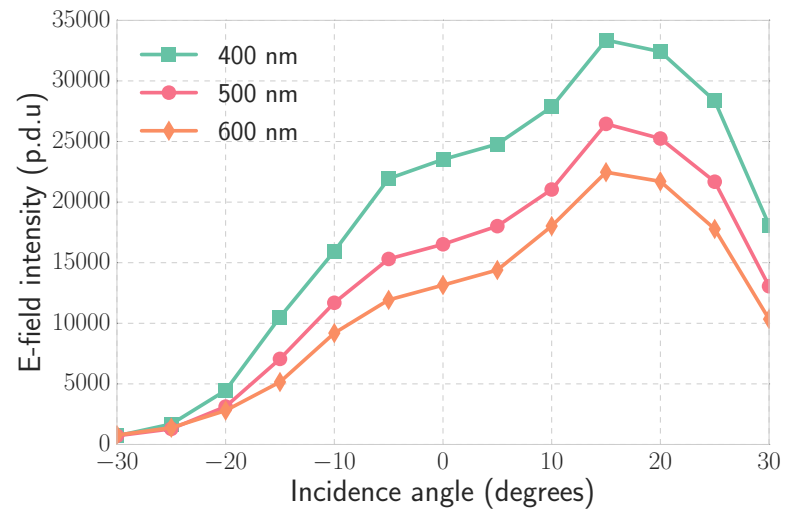

6.5.b: Response of horizontal QPC pixel (single pixel response).

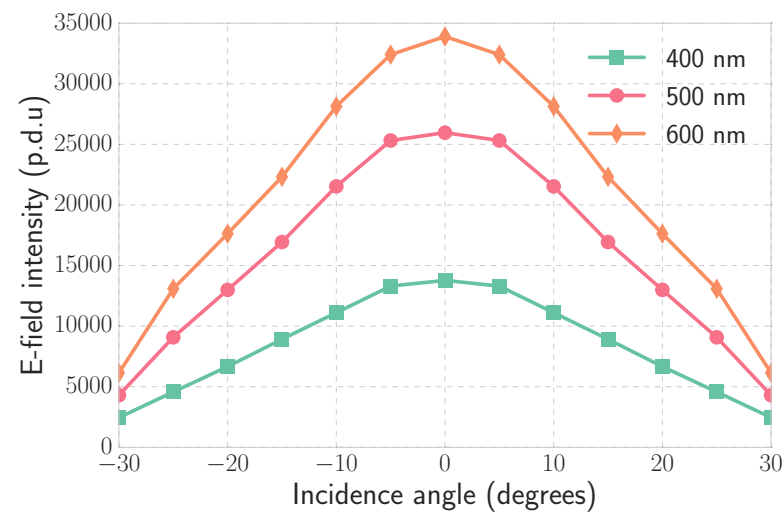

6.5.d: Response of horizontal polarization pixel.

Figure 6.5: Wavelength response of various angle sensitive pixel types.

In order to see the effect of wavelength changes to the QPC pixel we simulated a single QPC pixel by varying the wavelength from $400 \mathrm{~nm}$ to $600 \mathrm{~nm}$. Figure 6.5(b) shows the behavior. We note that shorter wavelengths produce stronger response than longer wavelengths as the effect of diffraction becomes stronger when the size of the metal masks become comarable to the wavelength. However the nature of the curves for angle variation remains the same as one would expect from a single QPC pixel. The same phenomenon can be noted with the horizontal multi-finger pixels (6.5(c)). 
The strength of the response produced by the polarization gratings for wavelength changes depends on the grating pitch. We observe a stronger polarization phenomenon when the incident wavelength, $\lambda$, is greater than twice the grating pitch, $\mathrm{d}(\lambda>2 \mathrm{~d})$. For the $200 \mathrm{~nm}$ pitch that we used, light of $600 \mathrm{~nm}$ wavelength produces stronger response as opposed to light of $400 \mathrm{~nm}$ (figure 6.5(d)).

\subsubsection{Polarization Sensitivity}

For the tests in this section we simulated angle variation (from $-30^{\circ}$ to $+30^{\circ}$ ) for various pixel types under two orthogonal polarization states (TE and TM). For TE polarized light, the electric field is parallel to the grating lines and for TM polarized light, the magnetic field is parallel to the grating lines.

Self-images of Talbot pixels at half Talbot depth formed by TE polarized light is twice as strong as compared to the TM polarized light [90]. As a result, TE polarized light produces a stronger response compared to the TM polarized light (figure 6.6(a)).

For horizontal multi-finger (figure 6.6(c)) pixels the response produced by TE polarized light is slightly greater than TM polarized light. This can be attributed to the same phenomenon as that of the Talbot pixels (effect of polarization on diffraction gratings), but since the grating pitch in MF pixels is larger than Talbot pixels, the effect is subdued.

QPC pixels show no change in response under varying polarization state, as expected (figure 6.6(b)).

As observed in the previous chapter, polarization pixels produce a strong response to TM polarized light (electric field orthogonal to grating lines) and a weak response to TE polarized light (electric field parallel to grating lines). This phenomenon is observed in figure $6.6(d)$. 


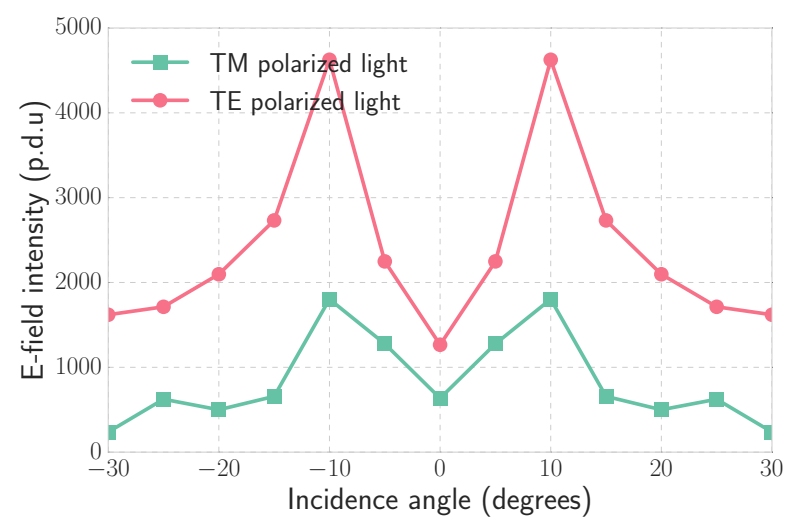

6.6.a: Response of Talbot $(\alpha=0)$ pixels.

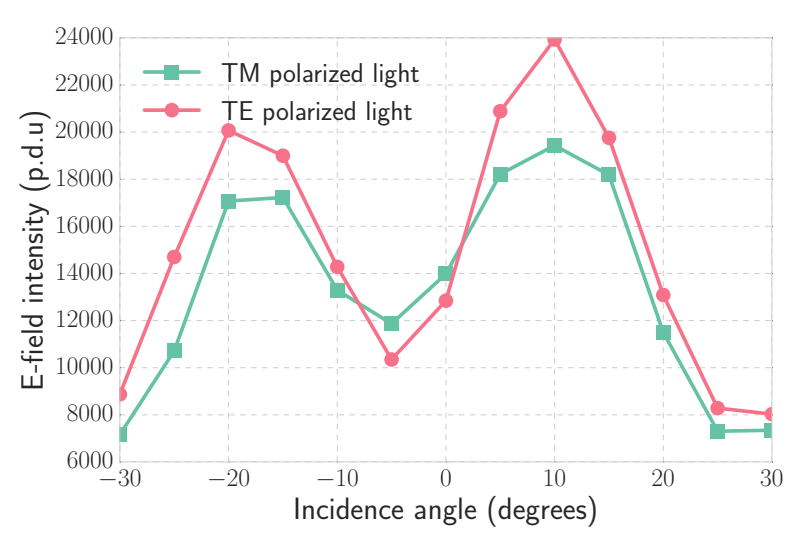

6.6.c: Response of horizontal multi-finger pixel.

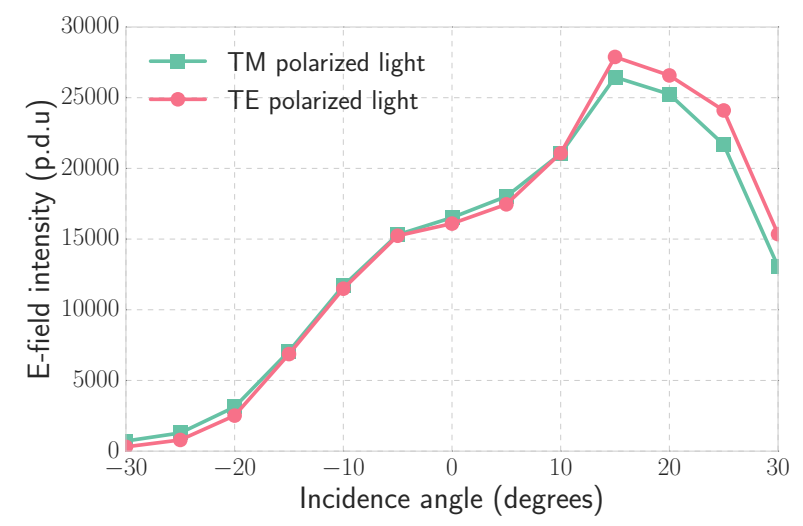

6.6.b: Response of horizontal QPC pixel (single pixel response).

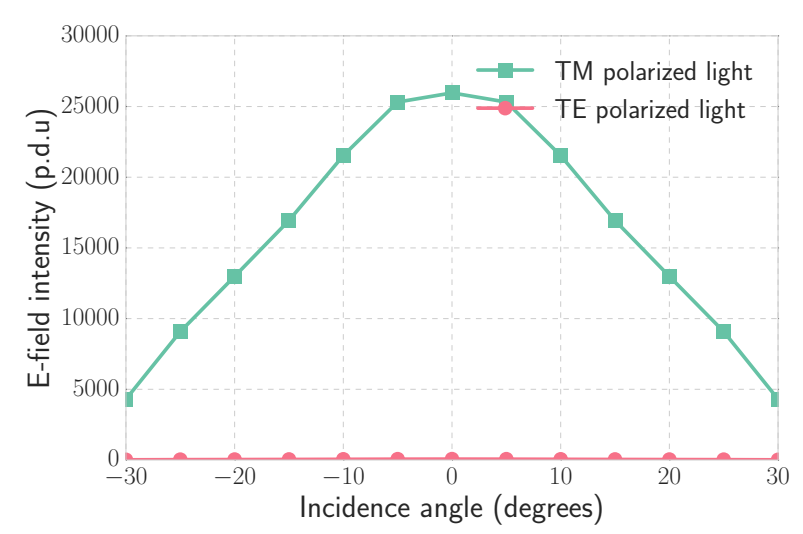

6.6.d: Response of horizontal polarization pixel.

Figure 6.6: Polarization response of various angle sensitive pixel types.

\subsubsection{Putting it all Together}

The parameter space of desirable characteristics for angle sensitive imaging is quite large. Deriving an optimized solution requires a multi-dimensional parameter optimization. However, based on the above analysis one could derive simple solutions by abstracting many of the finer parameters. For example, if one desires to have a very wide angle range and is only concerned with coarse angle detection, one could opt for enhanced QPC pixels. On the other hand, if one desires to capture very small variations in angle within a very narrow range one could opt for orthogonal MF pixels. The devised solution 
depends on the desired application.

\subsection{Applications of Angle Sensitive Imaging}

As was mentioned in chapter 4 we fabricated a CMOS image sensor in $0.35 \mu \mathrm{m}$ process. In the present section we explore some of the scenarios where the designed sensor could be utilized to simplify the imaging process.

\subsubsection{Fast Response Auto-Focus Systems}

A camera auto-focus system adjusts the camera lens to accurately focus on the subject of interest. Auto-focus systems fall into two broad categories - passive auto-focus systems and active auto-focus systems. The passive auto-focus systems [74] rely either on contrast detection or phase detection for determining the best lens position for good focus. The active auto-focus systems [12] [15] on the other hand, rely either on infrared or ultrasound to determine the distance to the subject and then estimate the focusing position of the lens for appropriate focus.

Figure 6.7 shows the auto focusing principle for an active auto-focus system. 'f' is the focal length of the lens. $\mathrm{p}$ and $\mathrm{q}$ are object and image distances. These variables can be related by the lens equation:

$$
\frac{1}{f}=\frac{1}{\mathrm{p}}+\frac{1}{\mathrm{q}}
$$

Active focusing systems measure p and estimate q, knowing f. They make the camera bulky and costly due to the additional light source. They are also not suitable when there are large movements in the scene and can only focus over a finite range. They are however particularly good under low light conditions and for featureless surfaces [39].

In contrast detection auto-focus (CDAF) [20], the camera lens is moved back-andforth until a point of maximum contrast is reached (figure 6.8). This maximum contrast 


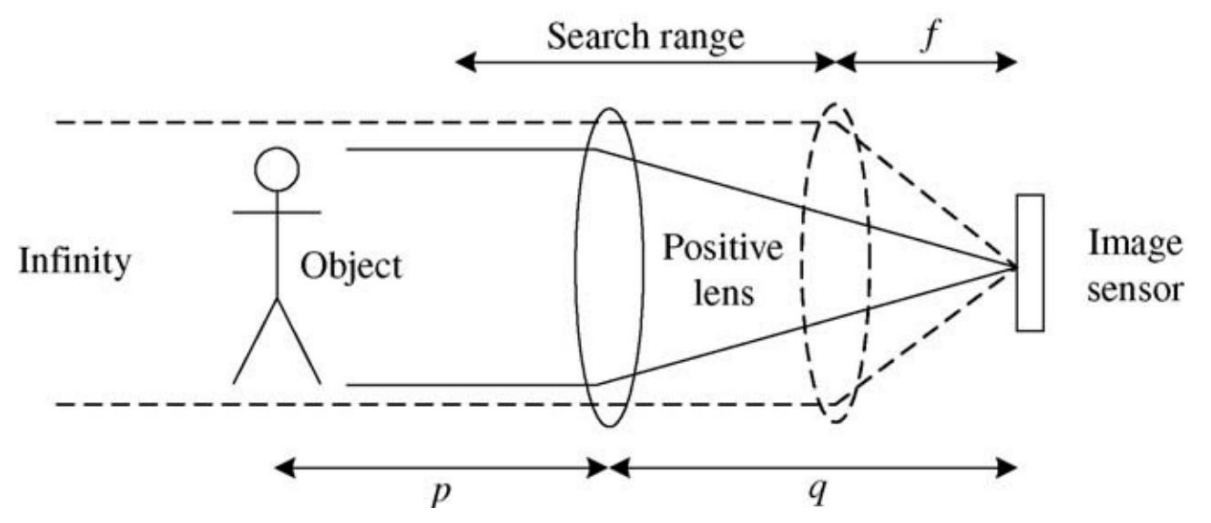

Figure 6.7: Principle of auto focus systems ( [14]).

point is the best focus location $\left(\mathrm{S}_{w}\right.$ in fig. 6.8). The algorithms for determining the sharpness function could either be based on spatial domain approaches [42], statistical approaches [99] [93] or frequency domain approaches [44] [9]. This iterative step typically takes a very short time before the camera locks into a sharp focus. For scenes where focus is difficult to achieve the camera gives up on trying to find the best focus location, a phenomenon known as "focus hunting" [2]. The CDAF systems take longer time in establishing the optimal focusing position and are also sensitive to noise [22].

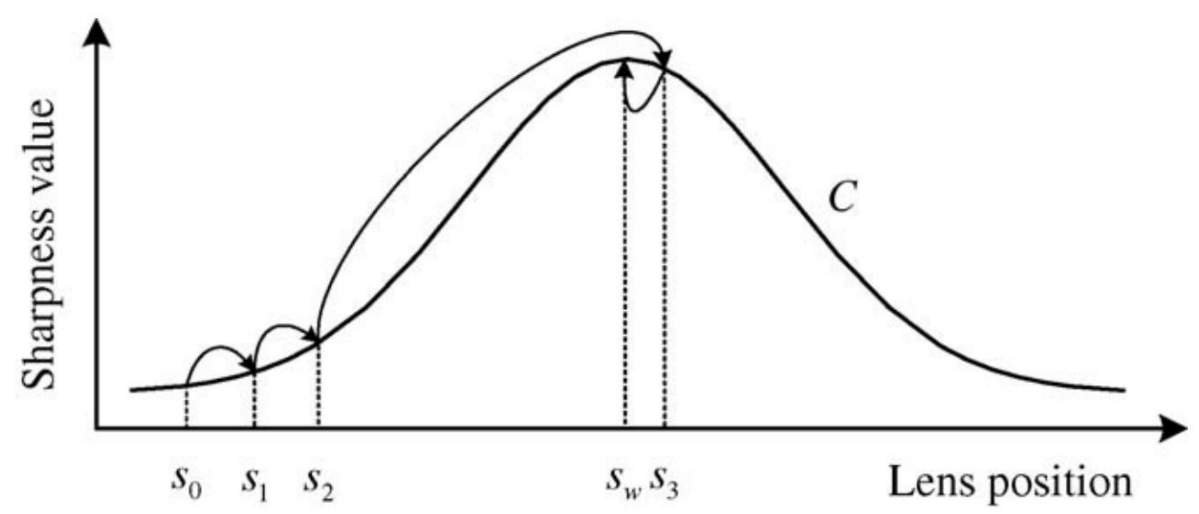

Figure 6.8: Principle of auto focusing (from [14])

In phase detection auto-focus (PDAF), the phase difference between light falling on different sensors is used as an aid for finding the correct focus position. Figure 6.9(a) 
(from [39]) shows the setup for a PDAF system inside the camera. Part of the light to the image sensor is split and redirected to two line sensors. As shown in figure 6.9(b) based on the focus position of the lens the phase difference between the two sensors vary. Based on the amount and nature of defocus (converging or diverging), the PDAF systems zone in on the correct lens position for optimal focus. Due to these properties PDAF systems turn out to be faster than CDAF and active auto-focusing techniques.
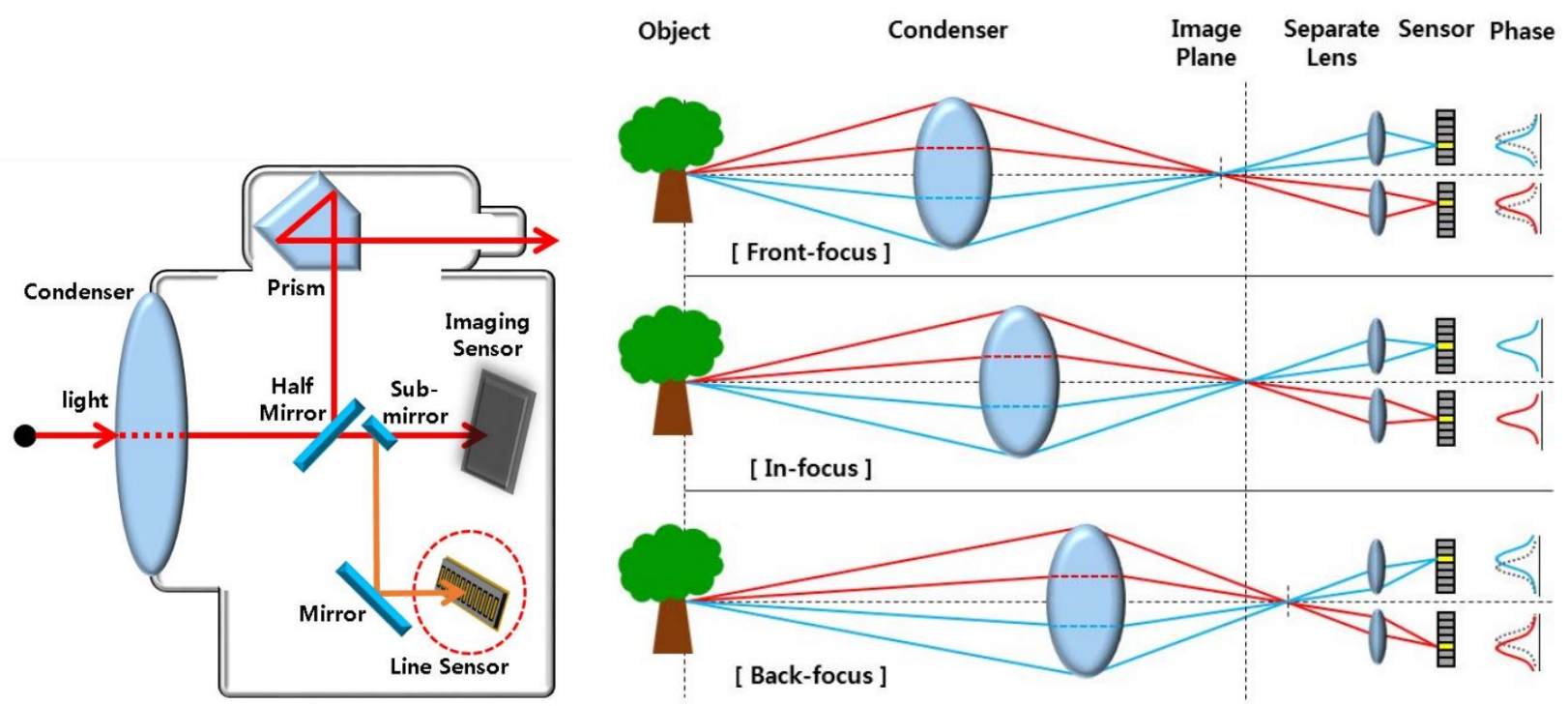

Figure 6.9: PDAF system (from [39])

As a consequence of the miniaturization of the fabrication processes, recent mobile phone cameras and some high end DSLRs incorporate a single sensor based phase detection auto focusing solution [73] [21]. A single sensor solution reduces the overall system cost while making the system more robust and easier to assemble. These special pixels know as PDAF pixels are scattered throughout the sensor array along with other conventional pixels and help to locally determine the phase difference for out of focus images.

The downside to a system using PDAF pixels is that they are sparsely spread throughout the pixel array and the local defocus measure is largely dependent on the presence 
of the PDAF pixels in vicinity of defocus.

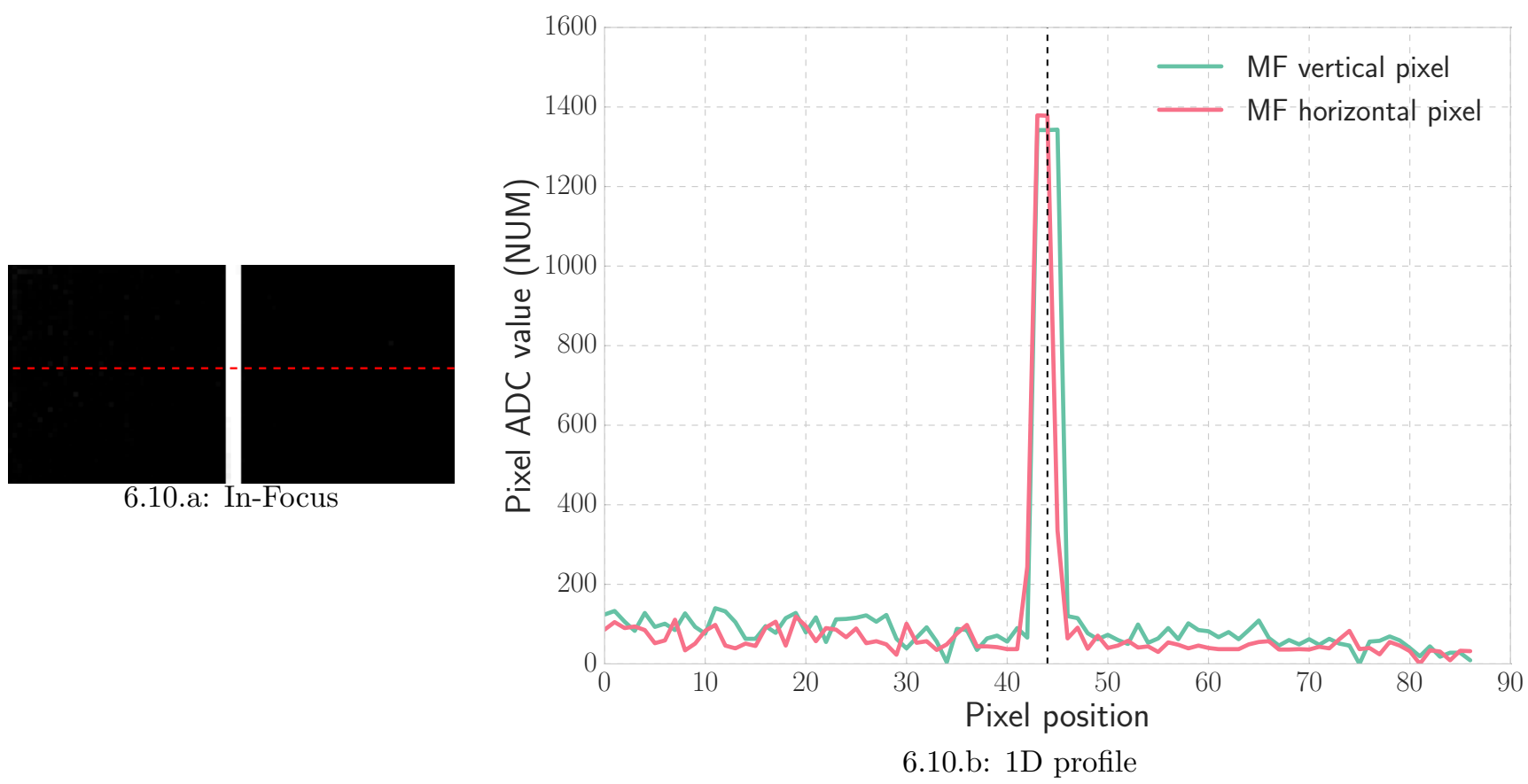

Figure 6.10: In-Focus image captured using MF sensor and its 1D profile.

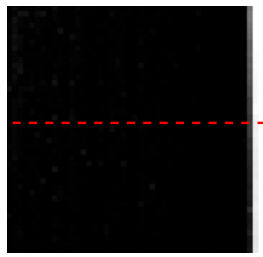

6.11.a: A

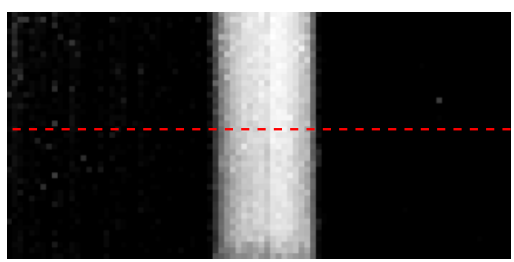

6.11.d: D

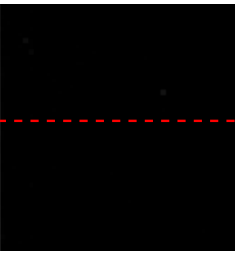

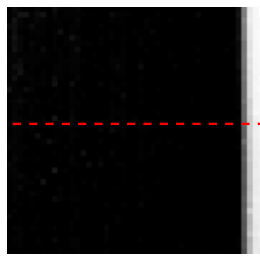

6.11.b: B

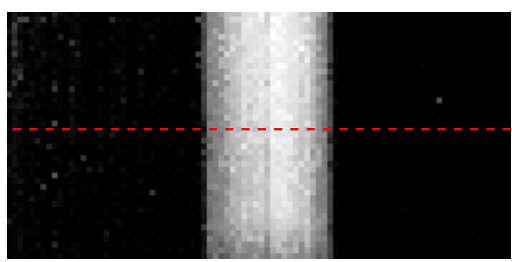

6.11.e: $\mathrm{E}$

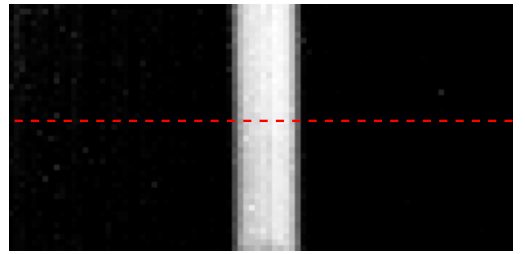

6.11.c: C

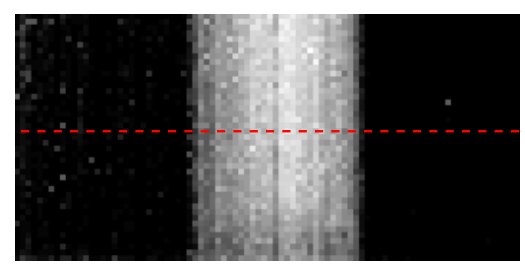

6.11.f: $\mathrm{F}$

Figure 6.11: Gradual change in image focus (pixels encounter converging angles).

The multi-finger pixel sensor that we presented earlier can detect the defocus measure at each pixel, thus providing faster and better guidance to camera auto-focus systems. 


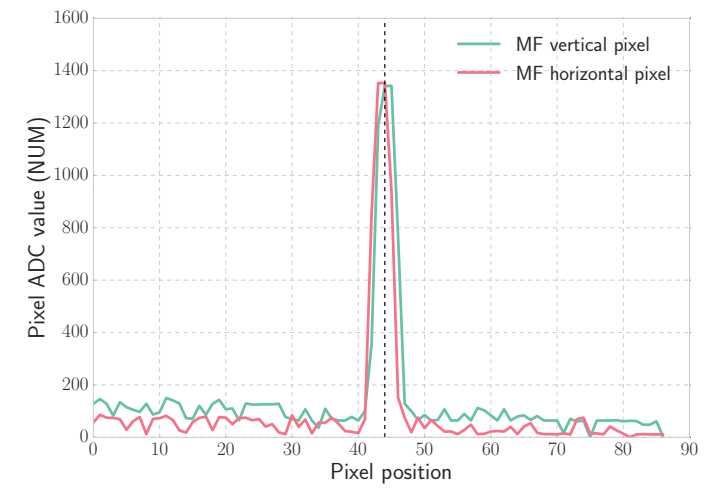

6.12.a: A

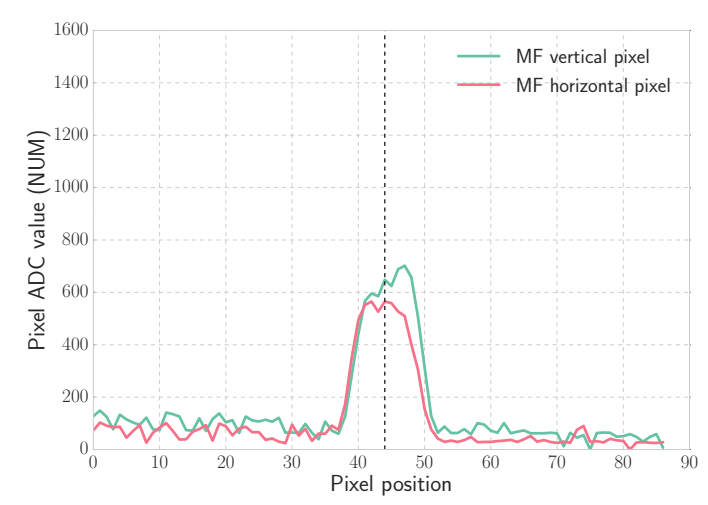

6.12.c: C

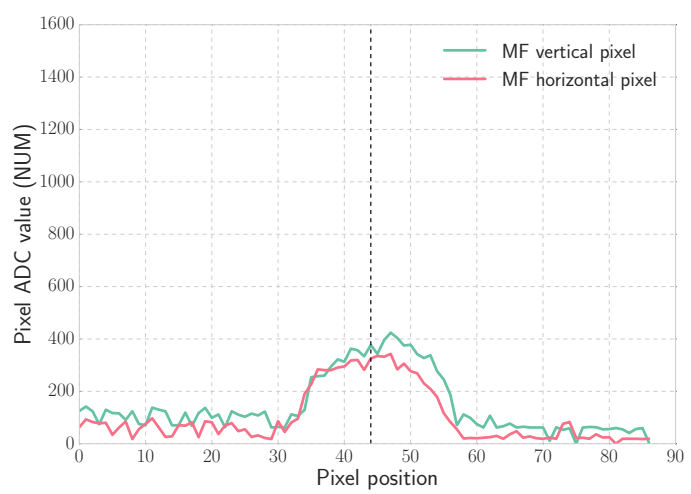

6.12.e: $\mathrm{E}$

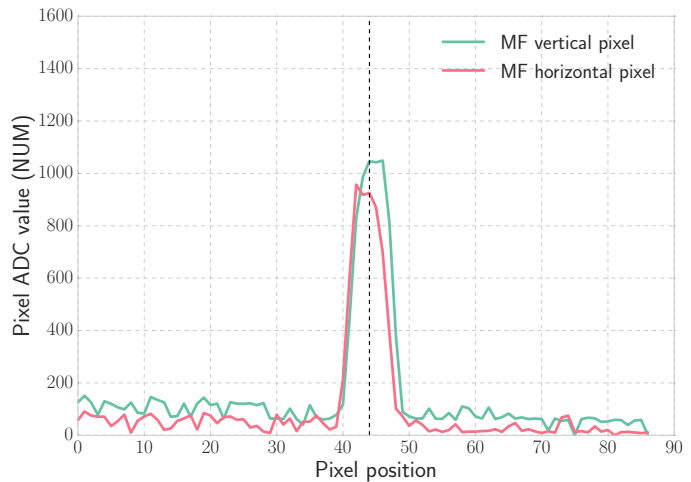

6.12.b: B

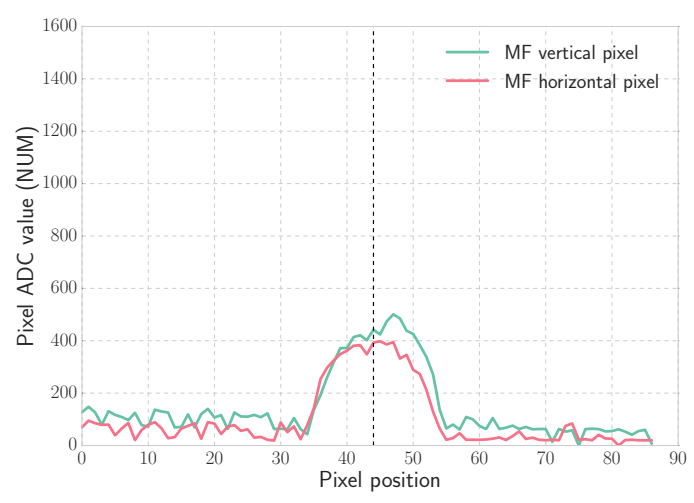

6.12.d: D

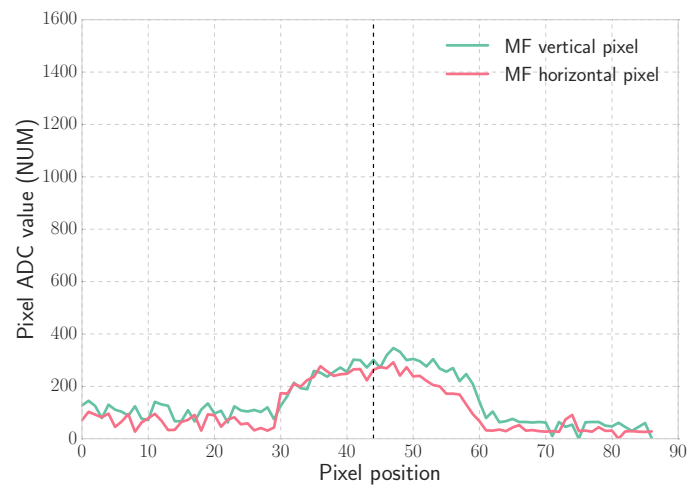

6.12.f: $\mathrm{F}$

Figure 6.12: 1D profile of figures 6.11(a) - 6.11(f)

Since the sensor consists of a pair of orthogonal MF pixels they can detect phase changes in only two orthogonal directions - horizontal and vertical. Horizontal MF pixels are sensitive to vertical angle variations and vice versa. A very simple algorithm for this sensor 


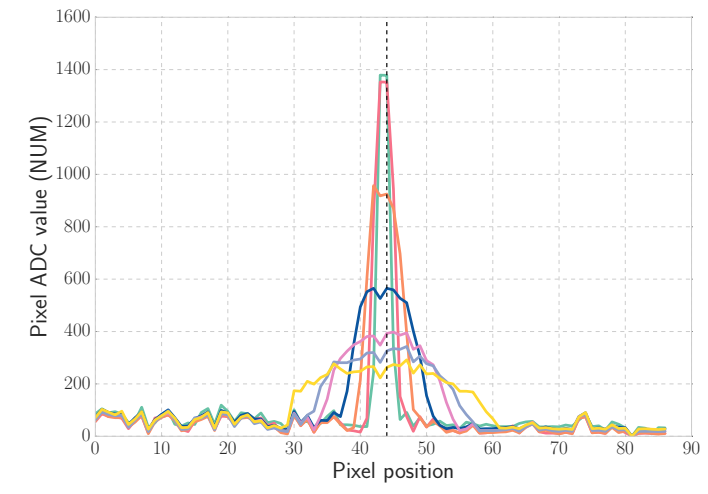

6.13.a: MF horizontal pixel response

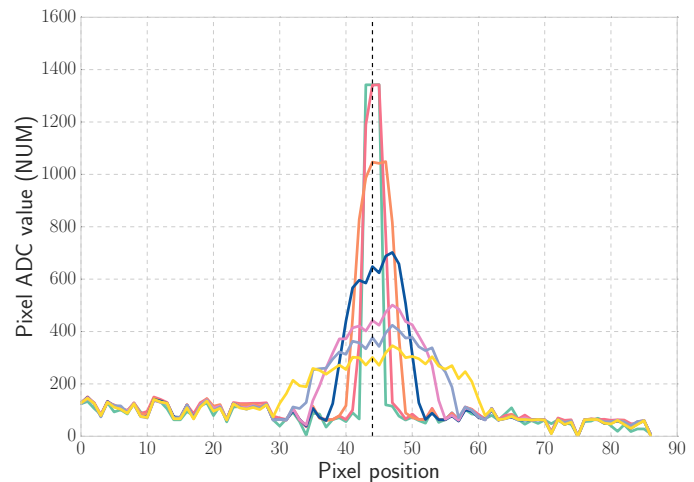

6.13.b: MF vertical pixel response

Figure 6.13: Plot highlighting the difference between horizontal and vertical MF pixel responses (figures are from 6.12)

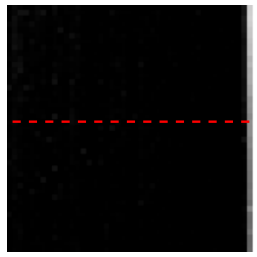

6.14.a: A

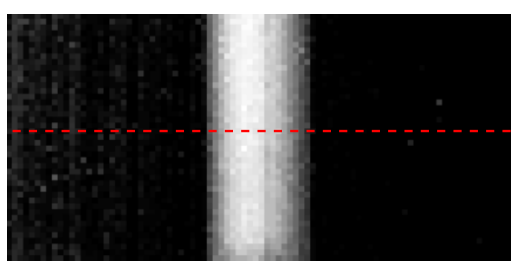

6.14.d: D

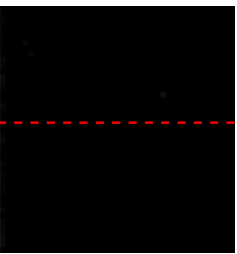

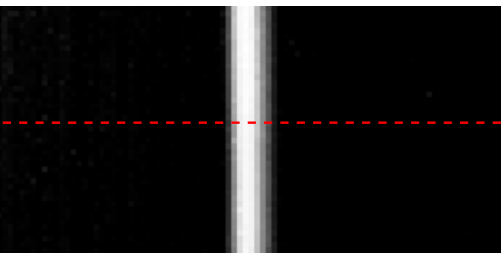

6.14.b: B

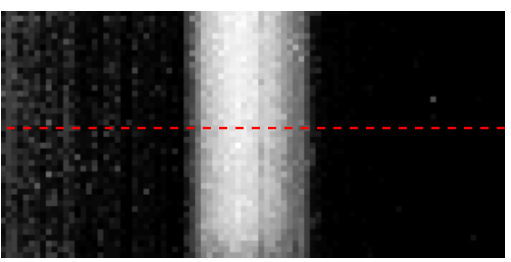

6.14.e: $\mathrm{E}$

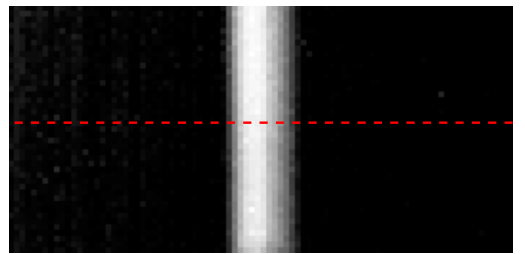

6.14.c: C

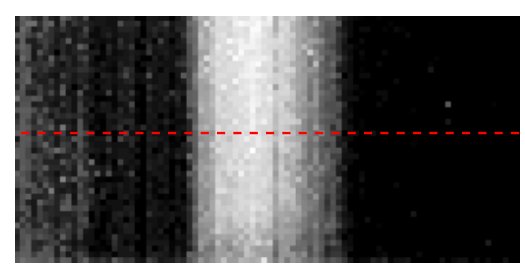

6.14.f: $\mathrm{F}$

Figure 6.14: Gradual change in image focus (pixels encounter diverging angles).

detects either local horizontal or vertical edges (one can employ canny edge detection) and then compares the slope of the two different MF pixel types.

Suppose the scene visible to the image sensor has vertical edges as shown in figure $6.10(\mathrm{a})$, the scene is in-focus and the response produced by the vertical and horizontal MF pixels are very similar as shown in figure $6.10(\mathrm{~b})$. For the $1 \mathrm{D}$ response in figure 6.10(b), the vertical pixel response is smaller than the horizontal pixel response due to unwanted metal layers in the periphery of the vertical pixels (due to asymmetry in pixel 

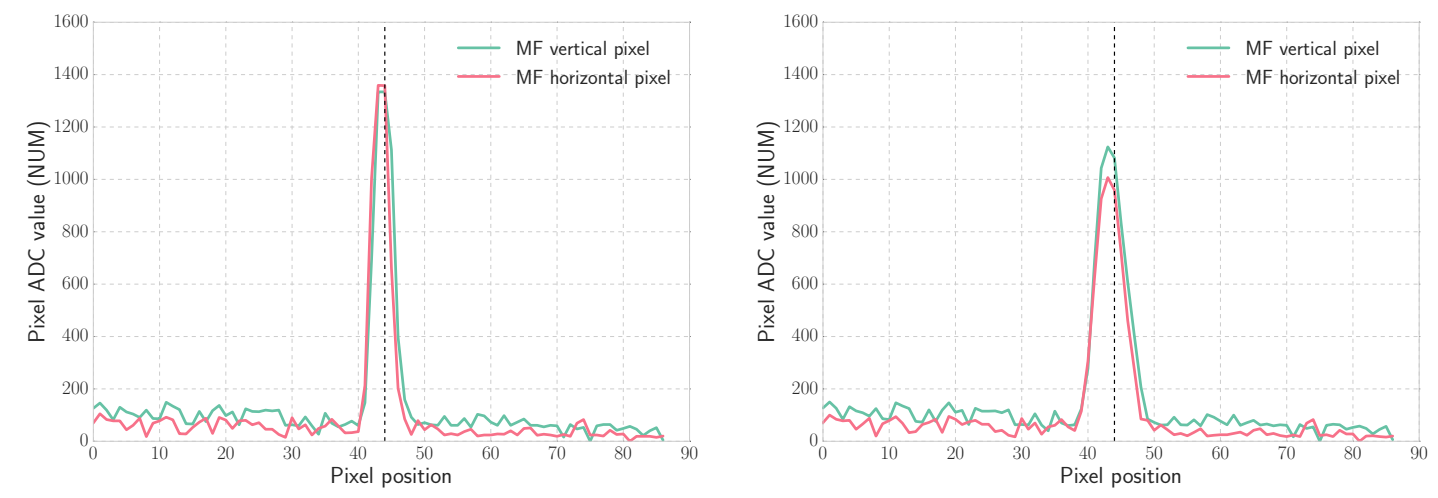

6.15.a: A

6.15.b: B
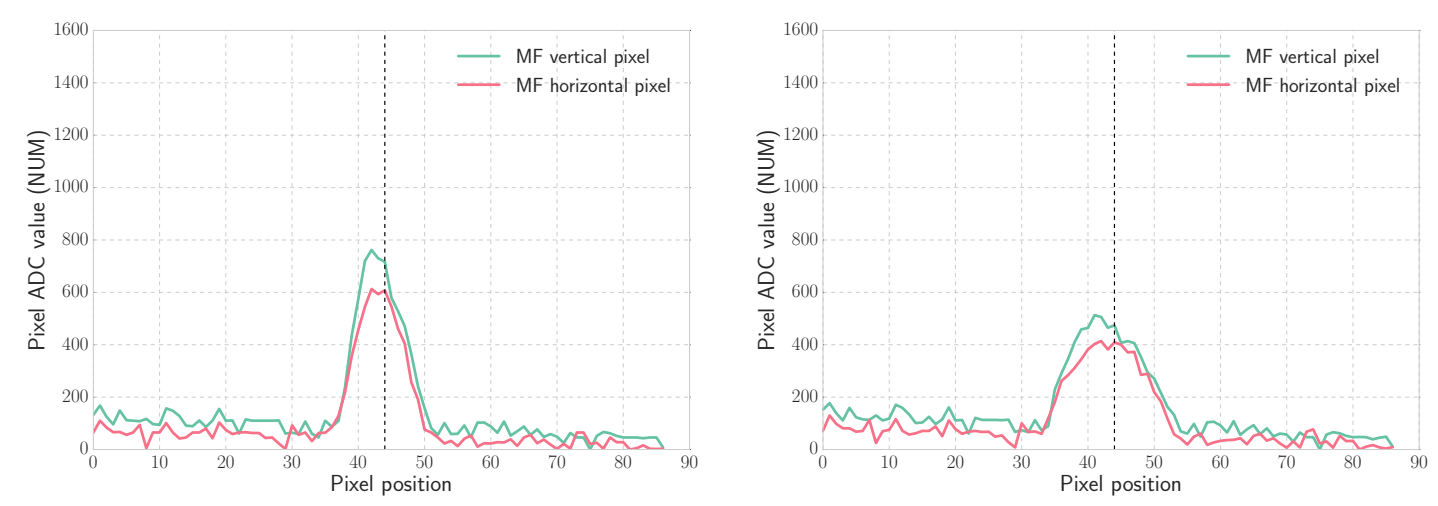

6.15.c: C

6.15.d: D

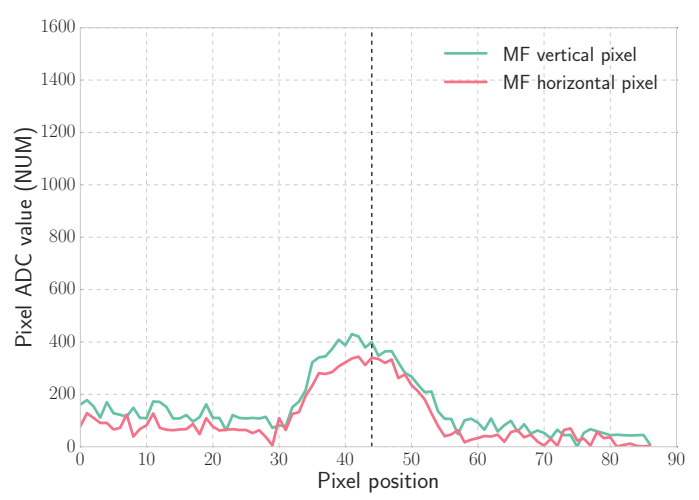

6.15.e: E

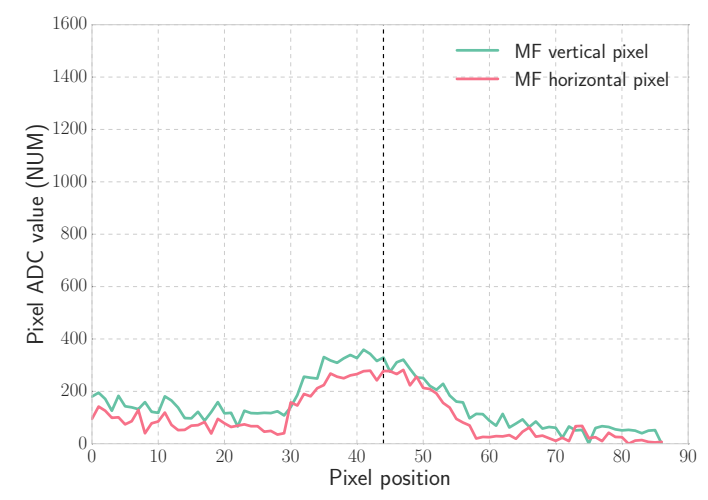

6.15.f: $\mathrm{F}$

Figure 6.15: 1D profile of figures 6.14(a) - 6.14(f)

design). However, one can notice that the slopes of the horizontal and vertical pixel responses are similar.

As we change the lens focus to focus in front of the object plane, the pixels encounter 


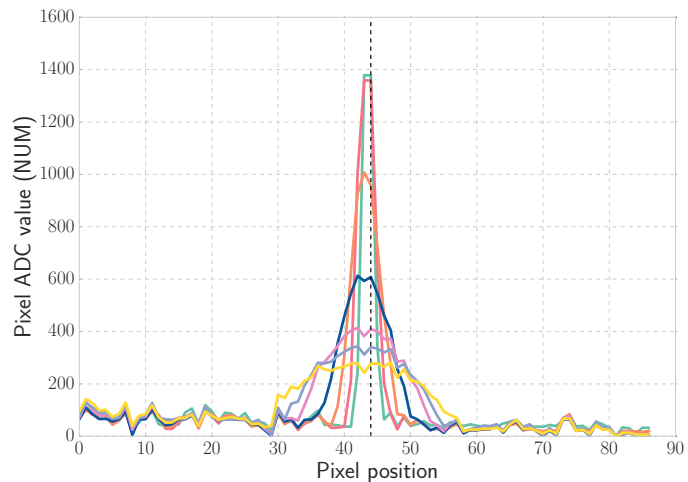

6.16.a: MF horizontal pixel response

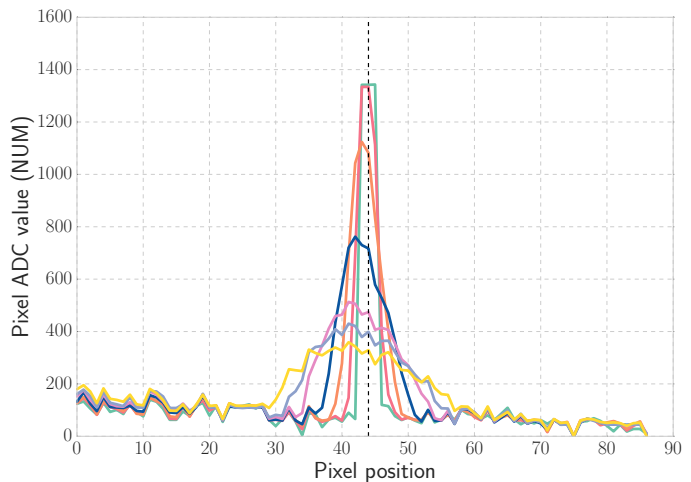

6.16.b: MF vertical pixel response

Figure 6.16: Plot highlighting the difference between horizontal and vertical MF pixel responses (figures are from 6.15).

converging angles at their focal plane. Since the imaged edge is vertical, only vertical pixels produce angle sensitive response. On the other hand, horizontal pixels produce a response that is similar to a conventional sensor, albeit, with reduced intensity due to the metal gratings. As we increase the defocus gradually from the smallest (figure 6.11(a)) to the largest (figure 6.11(f)), we see that the horizontal pixel response reduces in a predictable fashion (Gaussian profile), similar to a conventional sensor. The vertical pixel response on the other hand has a variable slope with a predisposition to the right. This can be observed for the individual focus settings from the 1D profiles in figure 6.12 or from the combined response in figure 6.13.

Contrast this with the images in figure 6.14 and their one dimensional line plot shown in figure 6.15. The lens focal plane for this case is beyond the object and the pixels encounter diverging angles at their focal plane. Once again examining the difference between the horizontal and vertical pixel responses (figure 6.15) we see that the slope of the horizontal pixels are almost consistent, whereas those for the vertical pixels gradually increase, with the peaks seemingly shifting to the left as the amount of defocus increases. This can be noted more clearly from figure 6.16. 
The results are in line with the angle variation tests that we reported in chapter 4 . As the image of the horizontal line is defocused, the vertical MF pixels encounter oblique light angles and produce their characteristic angle dependent response. The horizontal MF pixels too encounter oblique light angles, but since the change in the angle is along the pixel orientation their response does not vary with angle variation.

\subsubsection{Depth Estimation}

Light rays from a defocused image contains coarse depth information. When the object is in focus, all the rays terminate at the sensor placed at the lens focal plane. As the object moves toward the lens or away from it rays striking the sensor either converges or diverges. By determining the sign and magnitude of this convergence or divergence we can estimate the depth of the object from the lens. Figure 6.17 explains this concept in detail.

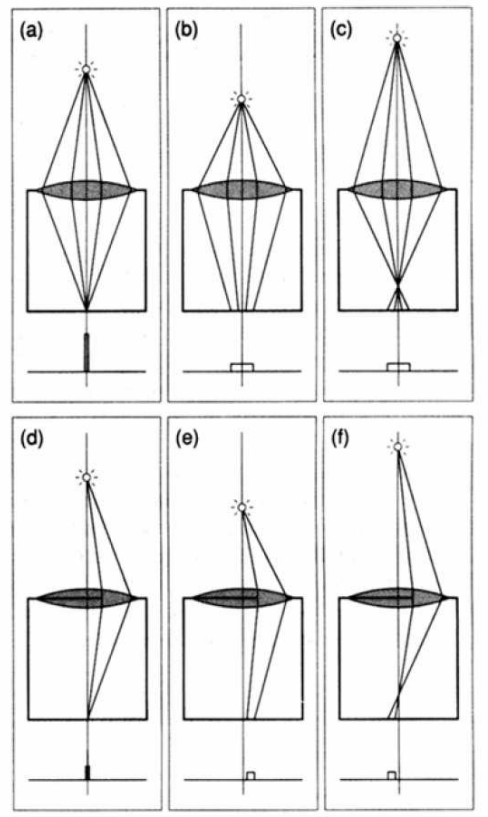

Figure 6.17: Figure to illustrate depth estimation process in multi-aperture image sensors [4].

Figure 6.17(a) shows a point object placed at the focal plane of the lens. It forms 
a sharp image of the point on the image sensor. The 1D representation at the bottom of figure $6.17(\mathrm{a})$ shows the intensity of light on the sensor plane as a function of pixel position. In this case, since the image formed is sharp, the intensity is highest, as shown by the thick line at the center.

In figure 6.17(b) the point object is moved closer to the lens, which results in a set of converging rays on the sensor plane. The image is blurred and the set of rays reaching the image plane has distinct directionality. The 1D representation shows a small rectangular box, which represents the intensity captured on the sensor because of the blurred image. In figure $6.17(\mathrm{c})$ the object is moved away from the lens resulting in a set of diverging rays on the sensor plane. The image is again blurred with the set of rays having distinct directionality. The 1D representation shows a rectangular box, similar to figure 6.17(b).

From the above explanation we can summarize that if the object is away from the plane of focus it forms a blurred image on the sensor plane and the set of rays have distinct directional information (figure 6.18).

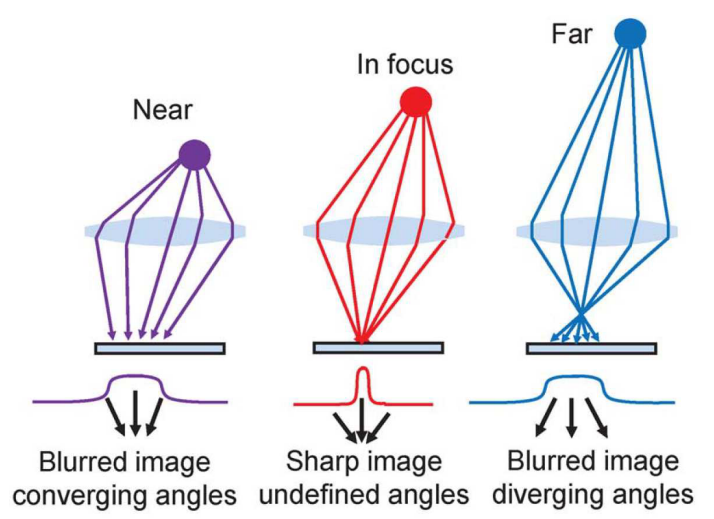

Figure 6.18: Directional information contained in the light rays when the object is "Near", "In-focus" and "Far" from the lens [88].

In order to understand the relationship between the amount of defocus (resulting from the distance of the object from the lens focal plane) and linear variation of image position on the sensor plane, let us insert an asymmetric aperture in front of the lens 
(Fig. 6.17(d), (e) and (f)) and observe the nature of image formed on the sensor plane. In Fig. 6.17(d), the point object is in focus and the resulting image formed is sharp but with reduced intensity because of the asymmetric aperture. In Fig. 6.17(e), the object is moved toward the lens which results in a blurred image on the sensor plane. But, since there is an asymmetric aperture, part of the rays are blocked from reaching the sensor plane. The 1D diagram shows that a small defocused image is formed on the right side of the sensor plane. Similarly, Fig. $6.17(\mathrm{f})$ shows that when the object is moved away from the focal plane of the lens, a defocused image is formed on the left side of the sensor plane. Hence, moving the object away from the lens focal plane shifts the image formed on the sensor plane to the left or right.

The above explanation gives an intuitive understanding of the information contained in a defocused image. We can use the multi-finger sensor to determine a coarse depth estimate as the multi-finger sensor can detect changes in light angles.

Figure 6.19 shows three vertical bars placed one behind the other. In figure 6.19(a), back bar is in focus and its 1D profile shows sharp edges for the back bar, with the sharpness (slope of horizontal MF pixel) degrading as we traverse from the back bar to the front bar. The vertical MF pixel shows no angle dependent response and degrades with defocus like a conventional intensity pixel. In figures 6.19(c) and 6.19(e) the focus in moved to the mid bar and then to the front bar. The 1D profiles of figure $6.19(\mathrm{~d})$ and figure 6.19(f) show the variation in the slope of the horizontal MF pixel response. The greater the difference in slope between the horizontal and vertical MF pixels, the larger is the distance of the object from the in-focus plane. Thus by examining the local pixel slope, we can quickly estimate the depth information contained in the scene.

\subsubsection{Post Capture Image Refocus}

Since multi-finger pixels are sensitive to angle variations we can capture the local angle information at each pixel level. Taken at a single pixel level this angle information is not of 


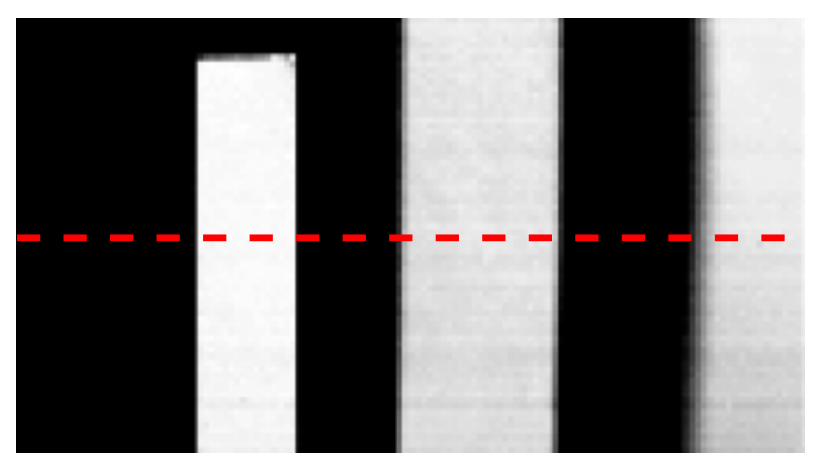

6.19.a: Back bar in focus

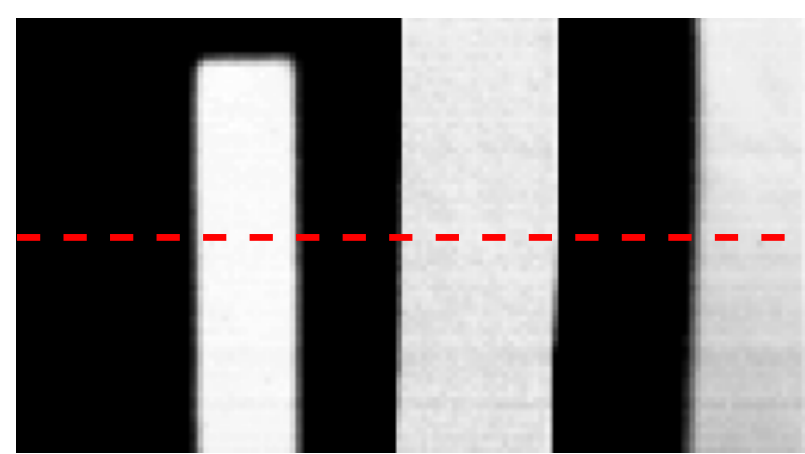

6.19.c: Mid bar in focus

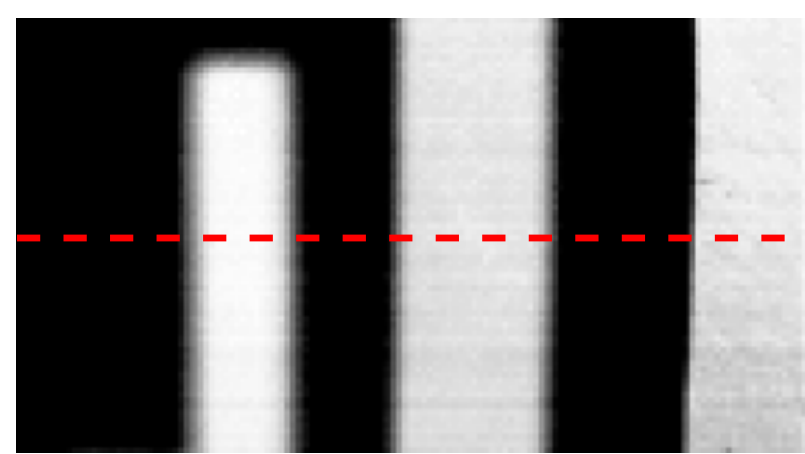

6.19.e: Front bar in focus

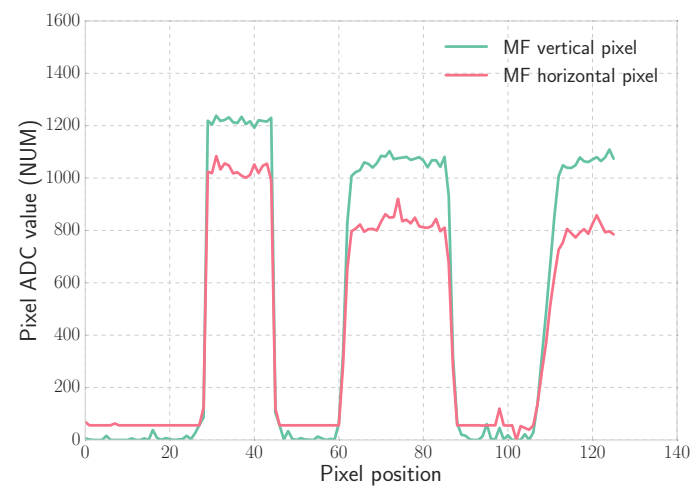

6.19.b: $1 \mathrm{D}$ profile for 6.19 (a)

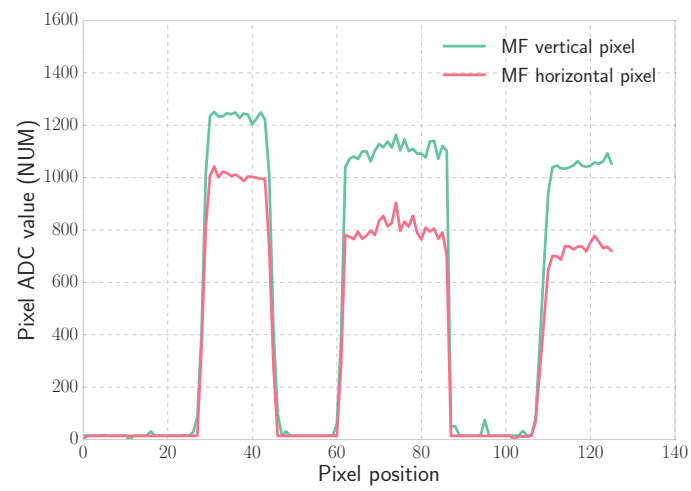

6.19.d: 1D profile for 6.19 (c)

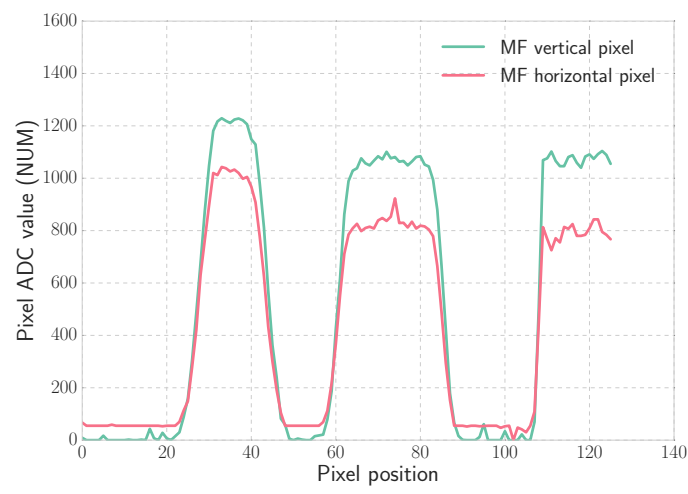

6.19.f: 1D profile for $6.19(\mathrm{e})$

Figure 6.19: Three white bars with one of the three in focus in each image and its 1D profile along the marked horizontal line.

much use, but when we consider it from the perspective of local angle variations in a small region, the nature of variations give a strong indication of the focus change. Furthermore, the angle sensitive pixels help to encode the mid-band frequency components of the 
captured image thereby making deconvolution a well-posed problem [64].

Image defocus could be considered as the convolution between the original latent image and the impulse response of the camera aperture function [45]:

$$
G(x, y)=I(x, y) * \mathrm{H}(\mathrm{x}, \mathrm{y})
$$

For a conventional image sensor this is an ill-posed problem to solve because both the impulse function $\mathrm{H}(\mathrm{x}, \mathrm{y})$ and the latent image $\mathrm{I}(\mathrm{x}, \mathrm{y})$ are unknowns. The image processing algorithms try to solve for this iteratively by trying to minimize an error function. Off-late the state of the art algorithms use image priors and natural image statistics to make a good approximation of the latent image and the impulse function.

One can approximate the impulse response by using a simple Gaussian profile:

$$
H(x, y)=\frac{1}{2 \Pi \sigma^{2}} e^{\frac{-\left(x^{2}+y^{2}\right)}{2 \sigma^{2}}}
$$

Here $\mathrm{x}$ and $\mathrm{y}$ are the pixel coordinates and $\sigma$ is the standard deviation of the Gaussian distribution. The Gaussian profile acts as a low pass filter thereby smoothing the image and irreversibly destroying the high frequency components. This causes ringing when one attempts to deconvolve the blurred image in order to extract the original image and degrade performance when one uses higher number of iterations to solve for the original image [54].

In order to see why deconvolution works with angle sensitive pixels let us examine the 1D nature of an image edge. Figure 6.20(a) shows the 1D profile of an image edge and the $1 \mathrm{D}$ profile of a Gaussian blur. As was discussed above, the convolution of the edge profile and the Gaussian blur results in the blur profile shown in Figure 6.20(b) for a conventional sensor. Since this is a slow smoothing over a few pixels, high frequency components of the edge profile is lost. Compare this with the profile for a MF (MultiFinger) sensor in which the change is very sharp over a few pixels, thereby preserving 
the mid-to-high frequency components. This aids greatly in deconvolution of the images using even a simple deconvolution algorithm such as the Richardson-Lucy algorithm [19].

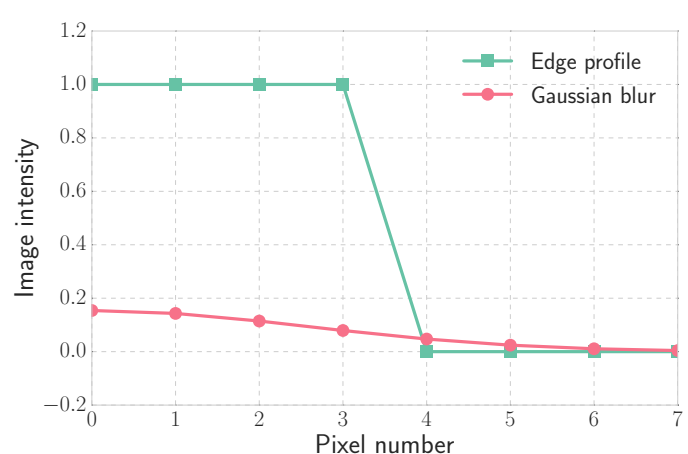

6.20.a: 1D sharp edge profile and 1D Gaussian blur profile

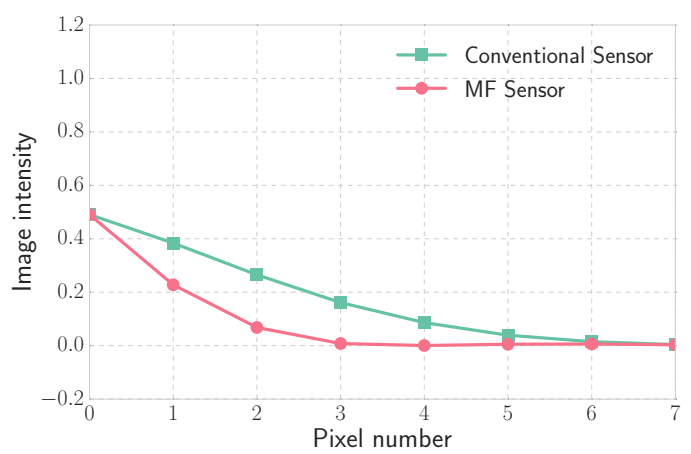

6.20.b: Blur profile of conventional sensor and MF sensor

Figure 6.20: Image blur profiles.

We use a simple Richardson-Lucy deconvolution algorithm to refocus the image. Figure 6.21(a) shows an image that was captured with a small defocus $(\sigma=9)$, its refocused version is shown in figure $6.21(\mathrm{~b})$.

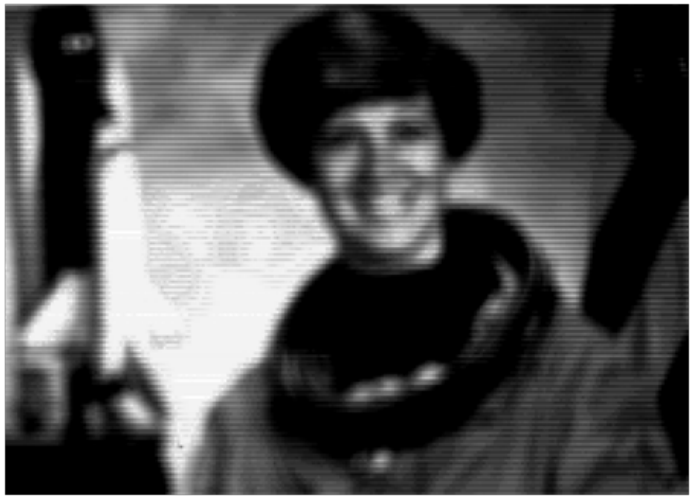

6.21.a: Defocused image

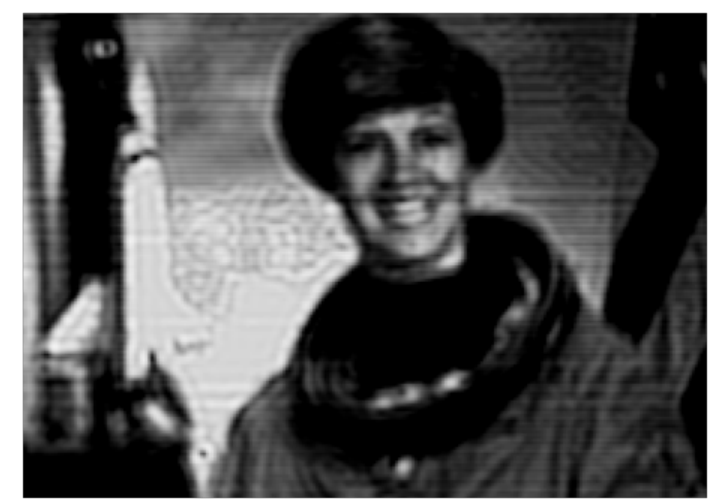

6.21.b: Refocused image

Figure 6.21: Image with a small amount of defocus and its refocused image $(\sigma=9)$.

Figure 6.22(a) shows an image that was captured with a significant defocus $(\sigma=13)$, and its refocused version is shown in figure 6.22(b). 


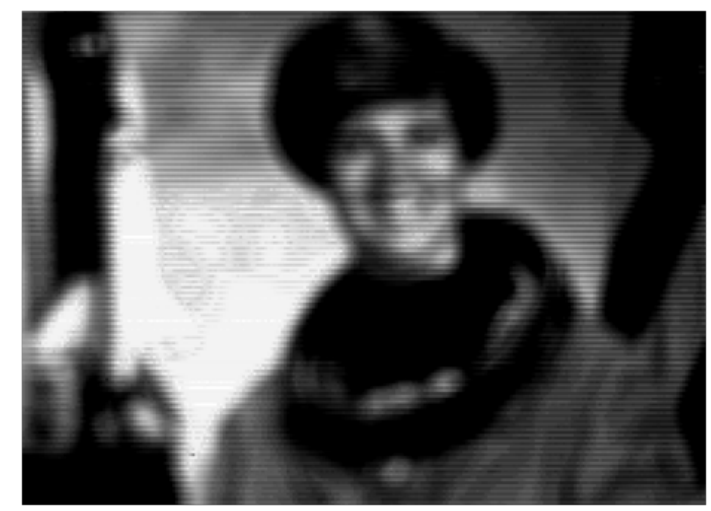

6.22.a: Defocused image

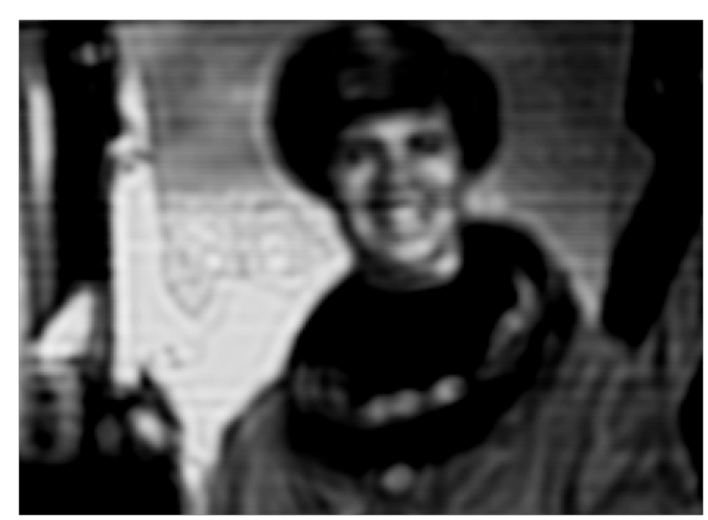

6.22.b: Refocused image

Figure 6.22: Image with a large amount of defocus and its refocused image $(\sigma=13)$.

In order to characterize the amount of blur in the image, we calculate the varianceof-laplacian (with the following kernel: $[[0,1,0],[1,-4,1],[0,1,0]])$ of the image as a defocus measure. This is listed in table 6.1. Lower values indicate higher defocus.

Table 6.1: Defocus and Refocus Measure

\begin{tabular}{|c|c|c|}
\hline Figure & $\begin{array}{c}\text { Kernel size } \\
(\sigma)\end{array}$ & Focus value \\
\hline Figure 6.21(a) & 9 & 834 \\
Figure 6.21(b) & 9 & 10458 \\
Figure 6.22(a) & 13 & 818 \\
Figure 6.22(b) & 13 & 3249 \\
\hline
\end{tabular}

Thus we conclude that by designing image sensors capable of capturing more information than that of the conventional sensors one can take advantage of the additional information for tasks such as post capture image refocus, depth estimation and fast auto-focus convergence as was demonstrated above. 


\section{Chapter 7}

\section{Conclusion and Future Research}

This chapter summarizes the many different threads that were existent throughout the thesis and emphasizes the need for angle sensitive imaging. It reinforces the need for a new sensor architecture for light field imaging and tries to evaluate the contributions of this thesis from a neutral standpoint. This chapter concludes by briefly touching upon some ideas that could be further pursued in this domain. This serves to whet the appetite of the reader and perhaps convince him/her that a great deal of interesting approaches could still be explored in this domain.

\subsection{Conclusion}

100 years of effort put into light field research is bearing fruit in the 21st century. Companies like Lytro, Raytrix, Pelican imaging and Light have put light field research into the hands of public. While the former two are based on plenoptic and focused plenoptic techniques, the later two use an array of image sensors, akin to a miniaturized version of the Stanford camera array. As the minimum feature size of the CMOS fabrication masks decrease for each generation of CMOS technology scaling, new techniques hitherto impossible to realize becomes feasible.

The techniques of angle sensitive imaging developed in this thesis builds on the earlier techniques of Talbot imaging that took advantage of the miniaturization afforded by 
CMOS technology scaling. The question that this thesis set out to address was - what is the minimum number of pixels that are required to capture the light field information?

Exploration of answers to this question took us through numerous challenges that led us to understand that the answer is not a simple one. One of our first attempts, the track-and-tune technique combines the angular information of the Talbot sensors with that of the quadrature pixel cluster (QPC) sensors to achieve a higher spatial resolution compared to the Talbot-only sensors. However, we noted that the Talbot sensors are sensitive to both incident light wavelength and polarization state along with the incident angle. The QPC sensors on the other hand had low angular sensitivity as they were based on the principle of metal shading. Although by combining the two we made use of the strengths of each technique, the number of pixels needed for this arrangement was still larger (13 pixels) than what we would have liked.

As we were experimenting with a bunch of other techniques we came upon polarization sensitive gratings that could turn a simple photodiode into a polarization detecting device. On close examination we learnt that apart from the polarization state of light, these gratings were also sensitive to incident light angles. This encouraged us to create a polarization-QPC sensor to detect both the incident light angle and the polarization state of light. As the polarization gratings need finer pitch, we had to use a $65 \mathrm{~nm}$ CMOS fabrication process to design this sensor and the devised detection scheme made use of 8 hybrid pixels to perform hybrid polarization-incident angle sensitive imaging.

In order to push the limits of angle sensitive imaging further we devised a new strategy to detect incident light angles. This sensor made use of multi-finger pixels that was based on the metal shading principle to detect the incident light angles. This technique achieved the highest spatial resolution among the incumbent techniques as it made use of only 2 pixels for angle detection. Although the angle sensitivity is not very high we proved that a sensor with very high spatial resolution with appreciable angular resolution can have many practical applications. 


\subsection{Summary of Contributions}

The main contributions that resulted from the work in this thesis are:

(i) Track-and-tune angle detection scheme that used only 13 angle detecting elements for local light angle detection. This was a $59 \%$ improvement from the state-of-theart technique of the time - the Talbot sensing technique.

(ii) Hybrid polarization-incident light angle detection scheme that could detect local polarization state of light as well the local angle information at a higher spatial resolution compared to the earlier techniques. This technique used 8 pixels for hybrid detection and achieved an improvement of $38 \%$ compared to the track-andtune angle detection scheme.

(iii) Multi-Finger angle detection pixels that was proposed as the final part of this work achieves a very high spatial resolution as it consumes only 2 pixels for local angle detection. This is an improvement of $75 \%$ compared to the earlier hybrid technique and facilitates practical application of angle sensitive imaging to real world scenarios.

\subsection{Future Research and Potential Applications}

We are just beginning to scratch the surface of what might be possible with the pixel level coding that was developed in this thesis. We have demonstrated that by capturing the $4 \mathrm{D}$ light field, i.e., by capturing the direction of light rays that terminate at a pixel, we can augment a conventional sensor to have additional capabilities. As impressive as it may be, we are just at the start of a new revolution in imaging. In what follows we try to unlock the full potential of the work carried out by examining ways in which we can expand its scope and range. 
(i) An unexplored part of the thesis is the creation of large depth of field images using the data collected by the angle sensitive pixels. This is quite straight forward by employing the digital photomontage technique proposed by Agarwala et al. [6]. (Applications: Imaging under low light conditions, imaging with object movement to extract spatio-temporal information).

(ii) This thesis only considers defocus that results due to improper focusing on the subject of interest and the those that can be approximated by a Gaussian blur profile. It does not take into account defocus that might be the result of camera shake or object/camera motion that can have complex blur profiles. Some computational photography techniques such as coded aperture and coded exposure are capable of handling variable blur profiles as they have attenuation masks that encode the blur profiles in terms of the masks and avoid zeros at high frequencies that blow up noise terms while performing deconvolution.

(iii) One way to improve the design presented in this work is to make the pixel size smaller than the one currently used. Smaller pixels lead to high pixel densities which provide better angular sampling and higher spatial resolution. Smaller pixel sizes result in lower light gathering capabilities for the photodiode and introduce additional complications in terms of longer exposure time (makes the camera more susceptible to camera shake) and larger aperture (makes the camera more susceptible to lens aberrations). While reducing the pixel size one must be aware of the additional complexities it involves and introduce techniques to circumvent the same. One could either use log pixels, gradient image capture scheme, multiple sensor exposures or other high dynamic range techniques to capture images with sufficient dynamic range while also using a smaller pixel. Higher spatial resolution 
and sampling could make the light field imagers attractive to professional photographers and might pave a wave for its inclusion in DLSR cameras and Mobile phone cameras.

(iv) As was demonstrated in one of the earlier chapters, polarization pixels have angle sensitivity in addition to polarization sensitivity. By using polarization pixels to detect the incident light angles we can also take advantage of their polarization detection capabilities. A sensor that can detect polarization can be used to aid in vision even in the presence of glare (useful for robotic surveillance) and to detect camouflaged objects (useful in military applications). 


\section{Publications}

\section{Journal Papers}

(1) Vigil Varghese, Shoushun Chen, "Angle Sensitive Imaging: A New Paradigm for Light Field Imaging," Optics Express, (In preparation).

(2) Vigil Varghese, Yu Hang, Shoushun Chen, "High Resolution Light Field Imaging through Monolithic Orthogonal Sinusoidal Angle Sensitive Pixel Image Sensor," IEEE Transactions on Multimedia, (In preparation).

(3) Vigil Varghese, Shoushun Chen, "Polarization Based Angle Sensitive Pixels for Light Field Image Sensors with High Spatio-Angular Resolution," IEEE Sensors Journal, vol.PP, no.99, pp.1-1, 2016.

(4) Vigil Varghese, Xinyuan Qian, Shouhsun Chen, Shen ZeXiang, Tao jin, Liang Guozhen and QiJie Wang, "Track-and-Tune Light Field Image Sensor," IEEE Sensors Journal, vol.14, no.12, pp.4372-4384, 2014.

\section{Conference Papers}

(1) Vigil Varghese, Jamal Lottier Molin, Christian Brandli, Shoushun Chen and Ralph Etienne-Cummings, "Dynamically Reconfigurable Silicon Array of Generalized Integrateand-Fire Neurons," Biomedical Circuits and Systems Conference (BIOCAS), 2015. 
(2) Hang Yu, Vigil Varghese, Xinyuan Qian, Menghan Guo, Shoushun Chen and Kay Soon Low, "An 8-Stage Time Delay Integration CMOS Image Sensor with On-Chip Polarization Pixels," International Symposium on Circuits and Systems (ISCAS), Lisbon, Portugal, May 2015.

(3) Vigil Varghese and Shoushun Chen, "Incident Light Angle Detection Technique Using Polarization Pixels,", IEEE Sensors Conference, Valencia, Spain, 2014.

(4) Vigil Varghese, Xinyuan Qian, Shoushun Chen and ZeXian Shen, "Linear Angle Sensitive Pixels for 4D Light Field Capture," 10th International SoC Design Conference (ISOCC), pp. 72-75, Busan, Korea, Nov. 2013. 


\section{Bibliography}

[1] Digital Photography Milestones from Kodak, 2014.

[2] Understanding Camera AutoFocus, 2016.

[3] Edward H. Adelson and James R. Bergen. The plenoptic function and the elements of early vision. In Computational Models of Visual Processing, pages 3-20. MIT Press, 1991.

[4] E.H. Adelson and J.Y.A. Wang. Single lens stereo with a plenoptic camera. Pattern Analysis and Machine Intelligence, IEEE Transactions on, 14(2):99-106, Feb 1992.

[5] Kraig Adler and JohnB. Phillips. Orientation in a desert lizard (uma notata): time-compensated compass movement and polarotaxis. Journal of Comparative Physiology A, 156(4):547-552, 1985.

[6] Aseem Agarwala, Mira Dontcheva, Maneesh Agrawala, Steven Drucker, Alex Colburn, Brian Curless, David Salesin, and Michael Cohen. Interactive digital photomontage. ACM Trans. Graph., 23(3):294-302, August 2004.

[7] A.G. Andreou and Z.K. Kalayjian. Polarization imaging: principles and integrated polarimeters. Sensors Journal, IEEE, 2(6):566-576, Dec 2002.

[8] Naoki Asada, Hisanaga Fujiwara, and Takashi Matsuyama. Edge and depth from focus. International Journal of Computer Vision, 26(2):153-163, 1998.

[9] J. Baina and J. Dublet. Automatic focus and iris control for video cameras. In Image Processing and its Applications, 1995., Fifth International Conference on, pages 232-235, Jul 1995. 
[10] Lior Blockstein and Orly Yadid-Pecht. Crosstalk quantification, analysis, and trends in cmos image sensors. Appl. Opt., 49(24):4483-4488, Aug 2010.

[11] Robert C. Bolles, H. Harlyn Baker, and David H. Marimont. Epipolar-plane image analysis: An approach to determining structure from motion. nternational Journal of Computer Vision, 1(1):7-55, 1987.

[12] B. Buttgen and P. Seitz. Robust optical time-of-flight range imaging based on smart pixel structures. IEEE Transactions on Circuits and Systems I: Regular Papers, 55(6):1512-1525, July 2008.

[13] Peter B. Catrysse, Xinqiao Liu, and Abbas El Gamal. QE reduction due to pixel vignetting in CMOS image sensors, 2000.

[14] Chih-Yung Chen, Rey-Chue Hwang, and Yu-Ju Chen. A passive auto-focus camera control system. Applied Soft Computing, 10(1):296 - 303, 2010.

[15] Filiberto Chiabrando, Roberto Chiabrando, Dario Piatti, and Fulvio Rinaudo. Sensors for 3d imaging: Metric evaluation and calibration of a ccd/cmos time-of-flight camera. Sensors, 9(12):10080, 2009.

[16] Michael Faraday. Thoughts on ray-vibrations. In Experimental Researches in Electricity, volume 3, pages 447-452. Cambridge University Press, 2012. Cambridge Books Online.

[17] K. Fife, A. El Gamal, and H.-S.P. Wong. A Multi-Aperture Image Sensor With 0.7 um Pixels in 0.11 um CMOS Technology. Solid-State Circuits, IEEE Journal of, 43(12):2990-3005, 2008.

[18] Keith G. Fife. Devices for Integrated Multi-Aperture Imaging. PhD thesis, Stanford, CA, USA, 2009.

[19] D. A. Fish, J. G. Walker, A. M. Brinicombe, and E. R. Pike. Blind deconvolution by means of the richardson-lucy algorithm. J. Opt. Soc. Am. A, 12(1):58-65, Jan 1995. 
[20] C. Florea and L. Florea. A parametric non-linear algorithm for contrast based auto-focus. In Intelligent Computer Communication and Processing (ICCP), 2011 IEEE International Conference on, pages 267-271, Aug 2011.

[21] S. Fujii, Y. Katsuda, and G. Yagyu. Image pickup device and image pickup element, March 2011. US Patent App. 12/735,639.

[22] M. Gamadia and N. Kehtarnavaz. Image restoration preprocessing for low light auto-focusing in digital cameras. In 2007 IEEE International Symposium on Consumer Electronics, pages 1-5, June 2007.

[23] Victor L. Gamiz. Performance of a four-channel polarimeter with low-light-level detection, 1997.

[24] Todor Georgeiv, Ke Colin Zheng, Brian Curless, David Salesin, Shree Nayar, and Chintan Intwala. Spatio-angular resolution tradeoffs in integral photography. In Proceedings of the 17th Eurographics Conference on Rendering Techniques, EGSR '06, pages 263-272, Aire-la-Ville, Switzerland, Switzerland, 2006. Eurographics Association.

[25] Todor Georgiev and Andrew Lumsdaine. Focused plenoptic camera and rendering. Journal of Electronic Imaging, 19(2):021106-021106-11, 2010.

[26] A. Gershun. The light field. Journal of Mathematics and Physics, 18:51-151, 1939.

[27] B. Girod and E.H. Adelson. System for ascertaining direction of blur in a rangefrom-defocus camera, October 23 1990. US Patent 4,965,442.

[28] Bernd Girod and Stephen Scherock. Depth from defocus of structured light, 1990.

[29] Raymon M. Glantz. Polarization analysis in the crayfish visual system. Journal of Experimental Biology, 204(14):2383-2390, 2001.

[30] Steven J. Gortler, Radek Grzeszczuk, Richard Szeliski, and Michael F. Cohen. The lumigraph. In Proceedings of the 23rd annual conference on Computer graphics and interactive techniques, SIGGRAPH '96, pages 43-54, New York, NY, USA, 1996. ACM. 
[31] W. L. L. Grimson. From Images to Surfaces: A Computational Study of the Human Early Visual System. MIT, Cambridge, MA, 1981.

[32] Craig W. Hawryshyn. Ultraviolet-polarization vision: its role in salmon navigation, 1997.

[33] Eugene Hecht. Optics. Addison Wesley, 4 edition, 2002.

[34] R. Hirsch. Seizing the light: a history of photography. McGraw-Hill, 2000.

[35] Uwe Homberg, Stanley Heinze, Keram Pfeiffer, Michiyo Kinoshita, and Basil el Jundi. Central neural coding of sky polarization in insects. Philosophical Transactions of the Royal Society of London B: Biological Sciences, 366(1565):680-687, 2011.

[36] Aaron Isaksen, Leonard McMillan, and Steven J. Gortler. Dynamically reparameterized light fields. In Proceedings of the 27th Annual Conference on Computer Graphics and Interactive Techniques, SIGGRAPH '00, pages 297-306, New York, NY, USA, 2000. ACM Press/Addison-Wesley Publishing Co.

[37] M. Ito and A. Ishii. Three-view stereo analysis. IEEE Transactions on Pattern Analysis and Machine Intelligence, PAMI-8(4):524-532, July 1986.

[38] Herbert E. Ives. A camera for making parallax panoramagrams. J. Opt. Soc. Am., 17(6):435-439, Dec 1928.

[39] Jinbeum Jang, Yoonjong Yoo, Jongheon Kim, and Joonki Paik. Sensor-based auto-focusing system using multi-scale feature extraction and phase correlation matching. Sensors, 15(3):5747, 2015.

[40] C. Koch, J. Oehm, J. Emde, and W. Budde. Light Source Position Measurement Technique Applicable in SOI Technology. Solid-State Circuits, IEEE Journal of, 43(7):1588-1593, 2008.

[41] C. Koch, J. Oehm, and A. Gornik. High precision optical angle measuring method applicable in standard CMOS technology. In ESSCIRC, 2009. ESSCIRC '09. Proceedings of, pages 244-247, 2009. 
[42] Eric Paul Krotkov. Active Computer Vision by Cooperative Focus and Stereo. Springer-Verlag New York, Inc., Secaucus, NJ, USA, 1989.

[43] Meenal Kulkarni and Viktor Gruev. A division-of-focal-plane spectral-polarization imaging sensor, 2012.

[44] S. Y. Lee, Y. Kumar, J. M. Cho, S. W. Lee, and S. W. Kim. Enhanced autofocus algorithm using robust focus measure and fuzzy reasoning. IEEE Transactions on Circuits and Systems for Video Technology, 18(9):1237-1246, Sept 2008.

[45] A. Levin, Y. Weiss, F. Durand, and W. T. Freeman. Understanding and evaluating blind deconvolution algorithms. In Computer Vision and Pattern Recognition, 2009. CVPR 2009. IEEE Conference on, pages 1964-1971, June 2009.

[46] Marc Levoy and Pat Hanrahan. Light field rendering. In Proceedings of the 23rd annual conference on Computer graphics and interactive techniques, SIGGRAPH '96, pages 31-42, New York, NY, USA, 1996. ACM.

[47] Shih-Schön Lin, Konstantin M. Yemelyanov, Jr. Edward N. Pugh, and Nader Engheta. Polarization-based and specular-reflection-based noncontact latent fingerprint imaging and lifting. J. Opt. Soc. Am. A, 23(9):2137-2153, Sep 2006.

[48] G. Lippmann. Epreuves reversible donnant la sensation du relief. J. Phys, $7: 821-825,1908$.

[49] Shuichi Makita, Yoshiaki Yasuno, Takashi Endo, Masahide Itoh, and Toyohiko Yatagai. Polarization contrast imaging of biological tissues by polarization-sensitive fourier-domain optical coherence tomography. Appl. Opt., 45(6):1142-1147, Feb 2006.

[50] Thuy A. Doan Marie Dacke and David C. O Carroll. Polarized light detection in spiders. Journal of Experimental Biology, 204(14):2481-2490, 2001.

[51] Parry Moon and Domina Eberle Spencer. Theory of the photic field. Journal of the Franklin Institute, 255(1):33 - 50, 1953. 
[52] Olivier Morel, Fabrice Meriaudeau, Christophe Stolz, and Patrick Gorria. Polarization imaging applied to 3d reconstruction of specular metallic surfaces, 2005.

[53] Francesc Moreno-Noguer, Peter N. Belhumeur, and Shree K. Nayar. Active refocusing of images and videos. ACM Trans. Graph., 26(3), July 2007.

[54] Ali Mosleh, J. M. Pierre Langlois, and Paul Green. Image Deconvolution Ringing Artifact Detection and Removal via PSF Frequency Analysis, pages 247-262. Springer International Publishing, Cham, 2014.

[55] M. Muller and R. Wehner. The hidden spiral: systematic search and path integration in desert ants, cataglyphis fortis. Journal of Comparative Physiology A, 175(5):525-530, 1994.

[56] K. Murari, R. Etienne-Cummings, N. Thakor, and G. Cauwenberghs. Which photodiode to use: A comparison of cmos-compatible structures. IEEE Sensors Journal, 9(7):752-760, July 2009.

[57] S.K. Nayar and Y. Nakagawa. Shape from focus. Pattern Analysis and Machine Intelligence, IEEE Transactions on, 16(8):824-831, Aug 1994.

[58] S.K. Nayar, M. Watanabe, and M. Noguchi. Real-time focus range sensor. In Computer Vision, 1995. Proceedings., Fifth International Conference on, pages 995-1001, Jun 1995.

[59] Ren Ng. Digital Light Field Photography. PhD thesis, Stanford, CA, USA, 2006.

[60] Ren Ng, Marc Levoy, Mathieu Brédif, Gene Duval, Mark Horowitz, and Pat Hanrahan. Light field photography with a hand-held plenoptic camera. Computer Science Technical Report CSTR, 2(11), 2005.

[61] Alex Paul Pentland. A new sense for depth of field. Pattern Analysis and Machine Intelligence, IEEE Transactions on, PAMI-9(4):523-531, July 1987.

[62] A. N. Rajagopalan and S. Chaudhuri. An mrf model-based approach to simultaneous recovery of depth and restoration from defocused images. Pattern Analysis and Machine Intelligence, IEEE Transactions on, 21(7):577-589, Jul 1999. 
[63] Ramesh Raskar. Computational Photography: Epsilon to Coded Photography, pages 238-253. Springer Berlin Heidelberg, Berlin, Heidelberg, 2009.

[64] Ramesh Raskar, Amit Agrawal, and Jack Tumblin. Coded exposure photography: Motion deblurring using fluttered shutter. ACM Trans. Graph., 25(3):795-804, July 2006.

[65] Lord Rayleigh. XXV. On copying diffraction-gratings, and on some phenomena connected therewith. Philosophical Magazine Series 5, 11(67):196-205, 1881.

[66] M. Sarkar, D. San Segundo Bello, C. Van Hoof, and A. Theuwissen. Integrated polarization analyzing cmos image sensor for material classification. Sensors Journal, IEEE, 11(8):1692-1703, Aug 2011.

[67] M. Sarkar, D. San Segundo Bello, C. Van Hoof, and A.J.P. Theuwissen. Integrated polarization-analyzing cmos image sensor for detecting the incoming light ray direction. Instrumentation and Measurement, IEEE Transactions on, 60(8):2759-2767, Aug 2011.

[68] Sarkar, M. and San Segundo Bello, D. and Van Hoof, C. and Theuwissen, A. Integrated polarization-analyzing cmos image sensor for detecting incoming light ray direction. In Sensors Applications Symposium (SAS), 2010 IEEE, pages 194-199, Feb 2010.

[69] Ashutosh Saxena, Jamie Schulte, and Andrew Y Ng. Depth estimation using monocular and stereo cues. In International Joint Conference on Artificial Intelligence, volume 7, 2007.

[70] Y.Y. Schechner, S.G. Narasimhan, and S.K. Nayar. Polarization-Based Vision through Haze. Applied Optics, Special issue, 42(3):511-525, Jan 2003.

[71] S. Sivaramakrishnan, A. Wang, P. Gill, and A. Molnar. Design and characterization of enhanced angle sensitive pixels. IEEE Transactions on Electron Devices, 63(1):113-119, Jan 2016. 
[72] S. Sivaramakrishnan, A. Wang, P. R. Gill, and A. Molnar. Enhanced angle sensitive pixels for light field imaging. In Electron Devices Meeting (IEDM), 2011 IEEE International, pages 8.6.1-8.6.4, Dec 2011.

[73] P. Sliwinski and P. Wachel. A simple model for on-sensor phase-detection autofocusing algorithm. Journal of Computer and Communications, 1(6):11-17, November 2013.

[74] M. Subbarao and J. K. Tyan. Selecting the optimal focus measure for autofocusing and depth-from-focus. IEEE Transactions on Pattern Analysis and Machine Intelligence, 20(8):864-870, Aug 1998.

[75] M. Subbarao, T.-C. Wei, and G. Surya. Focused image recovery from two defocused images recorded with different camera settings. Image Processing, IEEE Transactions on, 4(12):1613-1628, Dec 1995.

[76] H. F. Talbot. Facts relating to optical science. No. IV. Philosoph. Mag., 9(56):401-407, 1836.

[77] Markus Testorf, Jurgen Jahns, Nikolay A. Khilo, and Andrey M. Goncharenko. Talbot effect for oblique angle of light propagation. Optics Communications, 129(34):167 - 172, 1996.

[78] John C. Tuthill and Sonke Johnsen. Polarization sensitivity in the red swamp crayfish procambarus clarkii enhances the detection of moving transparent objects. Journal of Experimental Biology, 209(9):1612-1616, 2006.

[79] Y. Hang V. Varghese and S. Chen. High resolution light field imaging through monolithic orthogonal sinusoidal angle sensitive pixel image sensor (under review). IEEE Transactions on Multimedia, 2016.

[80] Vaibhav Vaish. Synthetic Aperture Imaging Using Dense Camera Arrays. PhD thesis, Stanford, CA, USA, 2007.

[81] V. Varghese and S. Chen. Angle sensitive imaging: A new paradigm for light field imaging (under review). Optics Express, 2016. 
[82] V. Varghese and S. Chen. Polarization-based angle sensitive pixels for light field image sensors with high spatio-angular resolution. IEEE Sensors Journal, 16(13):5183-5194, July 2016.

[83] V. Varghese, X. Qian, S. Chen, and S. ZeXiang. Linear angle sensitive pixels for 4d light field capture. In SoC Design Conference (ISOCC), 2013 International, pages 072-075, Nov 2013.

[84] V. Varghese, X. Qian, S. Chen, S. ZeXiang, T. Jin, L. Guozhen, and Q. J. Wang. Track-and-tune light field image sensor. IEEE Sensors Journal, 14(12):4372-4384, Dec 2014.

[85] A. Wang, P. Gill, and A. Molnar. Angle sensitive pixels in cmos for lensless 3d imaging. In 2009 IEEE Custom Integrated Circuits Conference, pages 371-374, Sept 2009.

[86] A. Wang, P. R. Gill, and A. Molnar. An angle-sensitive cmos imager for singlesensor 3d photography. In 2011 IEEE International Solid-State Circuits Conference, pages 412-414, Feb 2011.

[87] A. Wang and A. Molnar. Phase-based 3d optical flow sensors for motion detection. In Sensors, 2011 IEEE, pages 683-686, Oct 2011.

[88] A. Wang and A. Molnar. A Light-Field Image Sensor in $180 \mathrm{~nm}$ CMOS. Solid-State Circuits, IEEE Journal of, 47(1):257-271, 2012.

[89] A. Wang, S. Sivaramakrishnan, and A. Molnar. A 180nm cmos image sensor with on-chip optoelectronic image compression. In Proceedings of the IEEE 2012 Custom Integrated Circuits Conference, pages 1-4, Sept 2012.

[90] Albert Wang. Angle Sensitive Pixels For Integrated Light Field Sensing. PhD thesis, Ithaca, NY, USA, 2012.

[91] Albert Wang, Patrick Gill, and Alyosha Molnar. Light field image sensors based on the talbot effect. Appl. Opt., 48(31):5897-5905, Nov 2009. 
[92] T.H. Waterman. Polarization sensitivity. H.J.Altrum (ed.), Handbook of Sensory Physiology, 7, Part 6b: Vision of Invertebrates, Springer Verlag, Berlin:283-463, 1981.

[93] Chong-Yaw Wee and Raveendran Paramesran. Measure of image sharpness using eigenvalues. Information Sciences, 177(12):2533 - 2552, 2007.

[94] R Wehner, B Michel, and P Antonsen. Visual navigation in insects: coupling of egocentric and geocentric information. Journal of Experimental Biology, 199(1):129-140, 1996.

[95] Bennett Wilburn. High-performance Imaging Using Arrays of Inexpensive Cameras. PhD thesis, Stanford, CA, USA, 2005. AAI3153705.

[96] L.B. Wolff. Polarization-based material classification from specular reflection. Pattern Analysis and Machine Intelligence, IEEE Transactions on, 12(11):1059-1071, Nov 1990.

[97] Milin Zhang, Xiaotie Wu, Nan Cui, N. Engheta, and J. Van der Spiegel. Bioinspired focal-plane polarization image sensor design: From application to implementation. Proceedings of the IEEE, 102(10):1435-1449, Oct 2014.

[98] Milin Zhang, Xiaotie Wu, N. Engheta, and J. Van der Spiegel. A monolithic cmos image sensor with wire-grid polarizer filter mosaic in the focal plane. Electron Devices, IEEE Transactions on, 61(3):855-862, March 2014.

[99] Yani Zhang, Ying Zhang, and Changyun Wen. A new focus measure method using moments. Image and Vision Computing, 18(12):959 - 965, 2000.

[100] C. Zhou and S. K. Nayar. Computational cameras: Convergence of optics and processing. IEEE Transactions on Image Processing, 20(12):3322-3340, Dec 2011. 